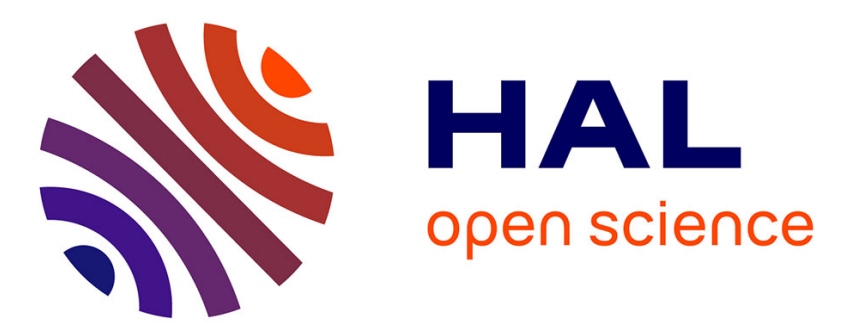

\title{
Artisanats spécialisés et inégalités sociales à l'aube de la métallurgie: les pointes de flèches de type armoricain dans le nord du Finistère
}

Clement Nicolas

\section{- To cite this version: \\ Clement Nicolas. Artisanats spécialisés et inégalités sociales à l'aube de la métallurgie: les pointes de flèches de type armoricain dans le nord du Finistère. Bulletin de la Société préhistorique française, 2011, 108 (1), pp.1-33. hal-02884407}

\author{
HAL Id: hal-02884407 \\ https://hal.science/hal-02884407
}

Submitted on 3 Jul 2020

HAL is a multi-disciplinary open access archive for the deposit and dissemination of scientific research documents, whether they are published or not. The documents may come from teaching and research institutions in France or abroad, or from public or private research centers.
L'archive ouverte pluridisciplinaire HAL, est destinée au dépôt et à la diffusion de documents scientifiques de niveau recherche, publiés ou non, émanant des établissements d'enseignement et de recherche français ou étrangers, des laboratoires publics ou privés. 
«Les pointes de flèches en silex, si admirables

de dessin et d'exécution, vont devenir la partie caractéristique d'une des périodes de l'âge du Bronze qui a disséminé ses tombeaux sur l'Armorique occidentale» (Martin, 1900, p. 167).

\begin{abstract}
Résumé
Au cours de l'âge du Bronze ancien (2300-1600 av. J.-C.), la Bretagne est le foyer d'une production particulière de biens de prestige : les pointes de flèches de type armoricain. Dans l'état actuel de nos connaissances, il s'agit du seul artisanat spécialisé de silex taillé qu'ait produit cette région de l'extrémité occidentale du continent européen. Objets détournés de leur fonction première, les pointes de flèches de type armoricain acquièrent une forte charge symbolique. Elles deviennent l'insigne d'une élite sociale à la tête de territoires fortement impliqués dans le développement de la métallurgie du bronze. Cet article présente une étude de 233 pointes de flèches de type armoricain provenant $d u$ nord du Finistère. Ces armatures sont hautement informatives sur la chronologie, la spécialisation artisanale et l'organisation sociale à l'âge du Bronze ancien en Bretagne. Elles amènent à reconsidérer certains aspects de la culture des Tumulus armoricains comme sa relation avec le Campaniforme, ses subdivisions chronologiques ou sa hiérarchie sociale.
\end{abstract}

Mots-clés

Pointes de flèches, silex, type armoricain, Campaniforme, âge du Bronze ancien, Bretagne, tombes, tumulus.

\begin{abstract}
In the Early Bronze Age (2,300-1,600 BC), Brittany was the place of a particular production of prestigious items: Armorican-type arrowheads. In the present state of our knowledge, this is the only specialized craft of knapped flint produced in this region of the western edge of continental Europe. Objects diverted from their primary function, Armorican-type arrowheads gain a strong symbolic meaning. They became the emblems of a social élite at the head of territories deeply involved in the development of bronze metalworking. This article presents a study of 233 Armorican arrowheads from Northern Finistère. These arrowheads are highly
\end{abstract}


informative regarding chronology, craft specialization and social organization during the Early Bronze Age in Brittany. They lead us to reconsider some aspects of the Armorican Tumulus culture, such as the link with the Bell Beaker phenomenon, chronological divisions and social hierarchy.

\section{Keywords}

Arrowheads, flint, Armorican type, Bell Beaker, Early Bronze Age, Brittany, graves, barrows.

\section{INTRODUCTION}

Jusqu'à présent, les pointes de flèches de type armoricain ont été retrouvées essentiellement en contexte funéraire, associées à des objets en bronze. Les tumulus dans lesquels elles étaient déposées sont attribués à l'âge du Bronze ancien d'après leur mobilier funéraire ainsi qu'une série restreinte de dates radiocarbones souvent bien imprécises (Briard, 1984). Le caractère funéraire des pointes de flèches est illustré par un corpus d'au moins 777 armatures déposées dans 40 tombes probablement individuelles, dont 754 pointes de type armoricain issues de 30 tombes (fig. 1 et tabl. 1). Rappelons que l'âge du Bronze ancien en Bretagne est connu essentiellement par ses monuments funéraires, quelques dépôts et de rares traces domestiques.
Les pointes de flèches de type armoricain appartiennent à un contexte culturel mal délimité dans le temps. D'un point de vue spatial, elles sont circonscrites à la partie occidentale du Massif armoricain, la BasseBretagne. S. Needham (2000, p. 171) donne une fourchette chronologique large pour l'âge du Bronze ancien de Basse-Bretagne, entre 2300 et 1600 av. J.-C.

D'un point de vue culturel, tous les éléments constitutifs de l'âge du Bronze ancien breton semblent préexister dans le Campaniforme : le développement de la sépulture individuelle sous tumulus (Briard, 1984, p. 191; Salanova, 2007), une différenciation qui s'opère dans les assemblages funéraires (Salanova, 1998 et 2007), l'accumulation et l'affichage de biens de prestige (Needham, 2000). L'assemblage funéraire campaniforme (gobelet en forme de cloche, poignard en cuivre, pointes de flèches, brassard d'archer, parure, outils; Salanova,

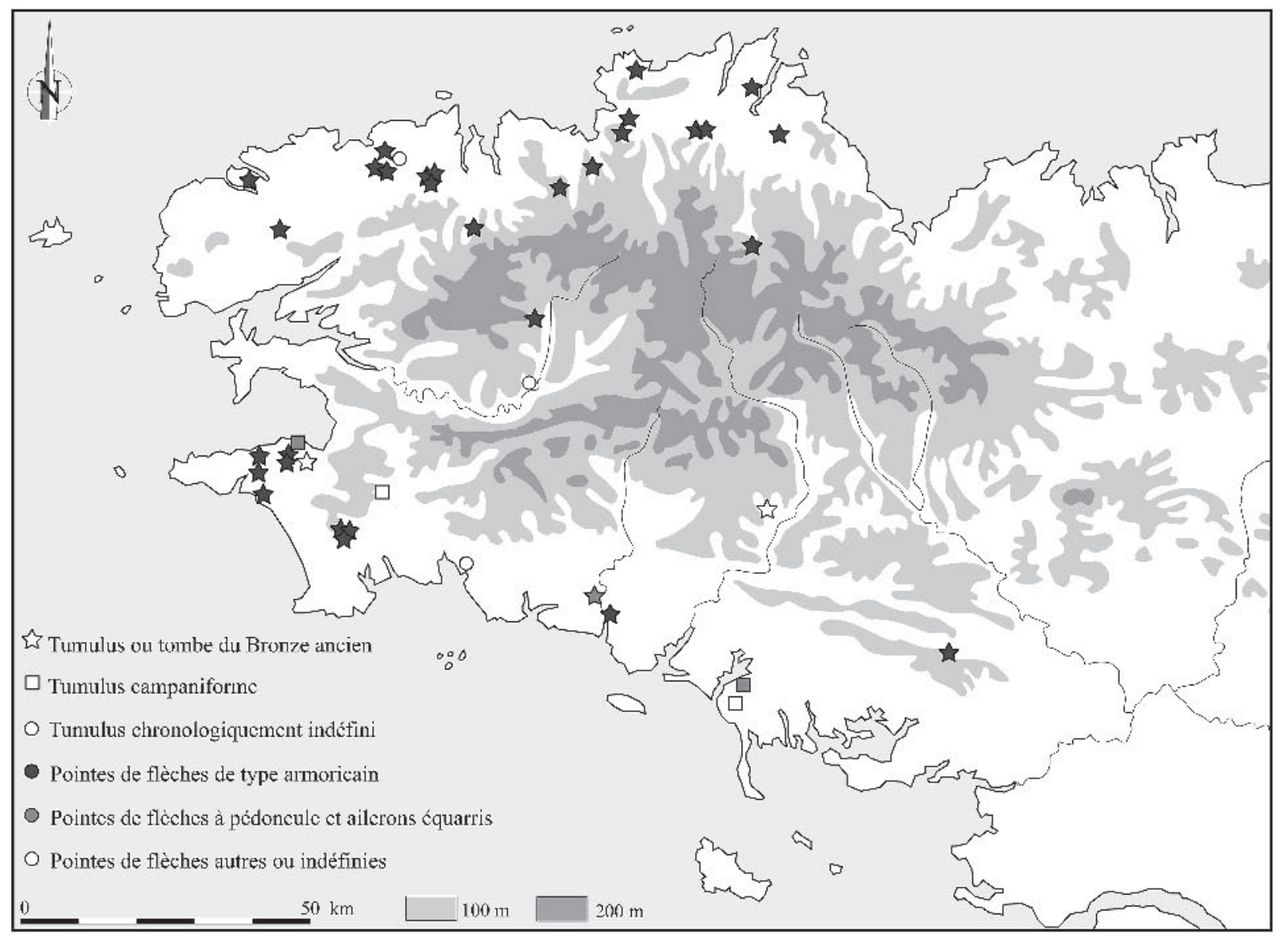

Fig. 1 - Carte de répartition des sépultures individuelles à pointes de flèches du Campaniforme et de l'âge du Bronze ancien en Bretagne. Fig. 1 - Distribution map of Bell Beaker or Early Bronze Age individual graves with arrowheads in Brittany. 
2007) paraît persister dans les tumulus de l'âge du Bronze breton (gobelet précieux, poignards en métal, pointes de flèches, brassard d'archer non fonctionnel, parure, outils; Needham, 2000; Needham et al., 2006).
Le changement transparaît dans l'abandon du gobelet céramique campaniforme et dans le développement de deux artisanats, la métallurgie du bronze et le façonnage des pointes de flèches de type armoricain.

\begin{tabular}{|c|c|c|c|c|c|c|c|c|c|c|}
\hline ipt. & Commmune & Site & 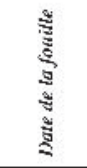 & 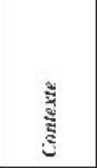 & 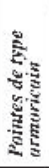 & 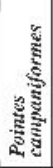 & 农 & 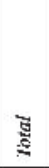 & $\begin{array}{l}\text { Attrihation } \\
\text { chrnotngique }\end{array}$ & Bithtiographie \\
\hline 22 & L.armiun & I +1 Molla & 1939 & Turnulus & 7 & & & 7 & Bromye ancien & Buller el Wialerbull, 1974 \\
\hline 22 & Pertos-Guirce & Crech-Perros & 1998 & Tumulus: & 25 & & & 25 & Bronze ancicn & Blanchet, 2005 \\
\hline 22 & Pleudariel & Vouslen-Brass & 1906 & Tumulus & 36 & & & 36 & Bronge ancien & Marl in el Prigent, 1907: Balquel, | 9499 \\
\hline 22 & Pleurniliau & Rumnédan & 19003 & Tummulus & 2.5 & & & 25 & Bronze anciern & Marlin, 19144b : Balque1, 19999 \\
\hline 22 & Prat & Tossen-Kergourognon & 1880 & Tumulus & 50 & & & 50 & Bronze ancicn & Prijent, 1881 \\
\hline 22 & Pral & Tixsen-Rugsumec: & $1900 \mathrm{r}$ & Tumulus & 46 & & & 46 & Bronye ancien & Marlin, 1914a \\
\hline 22 & Saint- $A$ dricn & Brun-Bras & 1974 & Tumulus & 45 & & & 45 & Bronze ancicn & Briard, 1978 \\
\hline 22 & Trémel & Porr-anr-Say). & 1875 & Tumulus & 29 & & & 29 & Brontre ancien & Prigent, I880 \\
\hline 22 & Trévérec & Toxsen-Maharii. & $189 \%$ & Tumulus & 20 & & & 20 & Bronye ancien & Marl in el Berthelot tu Chesmay, 1890 \\
\hline 29 & Bcuzcc-Cap-Sizun & Kerodou & 1890 & Tumulus & 3 & & & 3 & Bronze ancicn & Chatellicr, 1907 : Chaigncau-Norntand, 1994 \\
\hline 219) & Beu/ec-Cony & IzRhum & av. 1889 & Timbe & & & phinyentry & 2 & ? & Briarkl, 1984 \\
\hline 29 & Bourg Blane & Coatança & 1953 & Tumulus & 17 & & & 17 & Bronze ancicu & (Giot, 1953 : Nicolas, 2008 \\
\hline 29 & Cleder & Kergournadec & 1638 & Tumulus: & & & 2 & 2 & ? & Chatellicr, $1898 \mathrm{~b}$ \\
\hline 29 & I.anideleatu & Ty-Rsmú 2 & 1888 & Tumulus & & & 1 & 1 & $?$ & Halna tu Firelay, 1889 \\
\hline 29 & Lannilis & Prat-ar-Simon-Pella & 2005 & Tombe & 25 & & & 25 & Bronze ancicn & Le Goffie et Nallier, 2008 \\
\hline 29 & Plonèis & Conatjou-Gilas & v. 1886 & Tumnulus & & 2 & & 2 & Campanifisrme & (Maleellier, 1887 ; (Chaignemu-Normaml, 1944 \\
\hline 29 & Plonśour-Lanwem & Cosmaner & 1897 & Tumulus & 25 & & & 25 & Bronzc ancicn & Chatellicr, $1898 \mathrm{a}$; Chaigncau-Kormand. 1994 \\
\hline 29 & Plonćour-Lanwem & Fao-Youcn & 1897 & Tumulus: & 31 & & 1 & 32 & Bronze ancicn & Chatcllicr, $1898 \mathrm{a}$; Chaigncau-Kormand. 1994 \\
\hline 24 & ['onéoul-I.anvern & Kerhué-|3ras & 1875 & Tiumulus & 33 & & & 33 & Bronze ancien & Chatellier, $1880 \mathrm{a}$ et $1 \mathrm{ks} 0 \mathrm{~b}$ \\
\hline 29 & Plouhinec & Kutsandly & 1975 & Tumulus & 37 & & 1 & 38 & Bronze sucien & Briarkl. 1977b el 1984 : Briard et ol., 1982 \\
\hline 24 & I'oulinec & Lescongar & 19661 & Tumulus & 17 & & & 17 & Bronze ancien & B3riard, $196 \mathrm{~s}$ \\
\hline 29 & Plouigncau & Cazin & 1977 & Tumulus & 33 & & & 33 & Bromes ancicn & Briarl. 1977a o 1984; Giol. 1977 \\
\hline 24 & P'ounévez-1.nchrist & (ioarillach & 1867 & Tumulus & 22 & & & 22 & Bronge ancien & 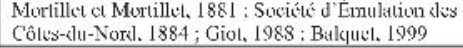 \\
\hline 24 & l'lounévez-1.nchrists & (iraenc 9 & av. 1489 & Tombe? & 3 & & & 3 & Bronze ancien & 1.e (intfic, 19 ML) \\
\hline 29 & Plouะun & K'monen & 1966 & Tumulus & 60 & & & 60 & Bronze ancion & Briarl. 1970a \\
\hline 29 & Ploux:nา & Kuruzunt: & 1898 & Tumulus & 11 & & & 11 & Bromze; ancicn & Kutrltril, 1898 ; Briarsl, 19706 \\
\hline 24 & l'louvorn & Lambader & ห. 1825 & Jombe & 1 & & & 1 & Bronze ancien & F réminville, 1832 ; Briard, $1470 \mathrm{~b}$ \\
\hline 29 & Plouye & Kurgućvartec & 1884 & Tumnulus & 24 & & & 24 & Bromze: ancion & Lukis, 1884 : Briard et ol, 1994 \\
\hline 24 & l'ouldergat & Kerlivit & 1892 & Jumulus & & & 1 & 1 & Bronze ancien & Halna du Frecay. 1893 ; Briard, 1984 \\
\hline 29 & Poullen-sur-Mar & Kเักาะทhir & 1883 & Tumulus & & 2 & & 2 & Campunifurm: & Chatcllicr, 1907 al archives: Sulunupra. 2000 \\
\hline 29 & Poullen-sur-Mcr & Kurvini Nurd & 1887 & Tumulus & 43 & & & 43 & Brume: ancicn & Hulne du Frelay, 1888 : Murtillk1. 1920 ; Briarcl. 1972 \\
\hline 24 & l'mullan-sur-Mer & Kervini Sud & 1887 & Tumulus & 9 & & & 9 & Bronze ancien & Halna du F recay, 1888 ; Mortillet, 1920 : Briard, 1972 \\
\hline 29 & Quimperle & Lothisi & 1843 & Tumulus & & 7 & & 7 & Bruma: ancicn & Taylur et ol., 1846 : Brisrd al Mohum. 1974 \\
\hline 29 & Sainl-Thégonnes & $\begin{array}{l}\text { Limbaluri'Creac'h- } \\
\text { Morvan }\end{array}$ & 1972 & Tumbe & 19 & & & 19 & Bronze ancion & Briarkl. 1984 \\
\hline 29 & Saint- $Y_{\text {๓qeąay }}$ & (iraenc 2:Liorzou 2 & ห. 1927 & Jumulus & 161 & & & 16 & Bronze ancien & Bénard, 1929 \\
\hline 56 & Bulz & Crubulz & r. 1864 & Tumnulus & & 1 & & 1 & Campenifurme: & Closmadeus, 1964 \\
\hline 56 & Elven & Cont-er-Gatt & v. 1413 & Jumulus & 28 & & & 28 & Bronze ancien & Marsille, 1913 \\
\hline 56 & Erkiwen & Sainl-Ginnain & \%. 1876 & Tumulus & & & 1 & 1 & Campentilurmi: & Gaillaril. 1892 ; Le Roulic, 1933 ; Briard, 1984 \\
\hline 56 & Guickl & Cruguel & 1890 & Tumzulus & 14 & & & 14 & Bromze suncicn & Le Ponlois. 1890 \\
\hline \multirow[t]{2}{*}{56} & M:lrand & Sainl-Fiatre: & \%. 1898 & Tumulus & & & 2 & 2 & Bromze : stcion & $\begin{array}{l}\text { Aveneau de la Grancière, } 1898 \text { : Needham el al., } \\
2006\end{array}$ \\
\hline & & & & Tolal & 754 & 12 & 10 & 777 & & \\
\hline
\end{tabular}

Tabl. 1 - Inventaire des tombes individuelles à pointes de flèches du Campaniforme et de l'âge du Bronze ancien en Bretagne. Table 1 - Inventory of Bell Beaker and Early Bronze Age individual graves with arrowheads in Brittany. 
La finesse de ces pointes de flèches de type armoricain, relevée depuis le $\mathrm{xIX}^{\mathrm{e}}$ siècle, est telle qu'il est impossible de reproduire un dessin exact et précis d'une pièce à l'œil nu et que la main des meilleurs tailleurs actuels n'arrive pas à une telle minutie. La standardisation et la production en série de ces armatures nous permettent-elles d'identifier leurs auteurs? Peut-on percevoir des variations techniques ou morphologiques qui traduiraient leur mode de fabrication? Est-il possible de localiser leur lieu d' origine? Peut-on aboutir à une histoire de ces pointes de flèches ?

Les séries de pointes de flèches ne sont pas distribuées uniformément dans l'espace, mais sont conditionnées par la vie et le lieu d'inhumation de leurs ultimes destinataires. À partir de là, est-ce que leur présence, ou leur absence, de certains territoires peut nous informer sur leurs destinataires? Et pourquoi d'autres individus n'ont-ils pas été enterrés avec des pointes de flèches? Chaque série nous renseigne sur au moins deux personnes - l'artisan et le destinataire - et leurs espaces. La pointe de flèche devient un vecteur pour comprendre et reconstituer les sociétés de l'âge du Bronze ancien en Basse-Bretagne.

\section{LES SÉPULTURES À POINTES DE FLĖCHES}

\section{Historique des recherches}

Nous nous contentons de rappeler ici quelques faits illustrant la nature du corpus étudié. Pour un historique détaillé, plusieurs références peuvent être consultées (Briard, 1984; Giot et al., 1995 ; Giot, 1997; Balquet, 2001 ; Coativy, 2006). La plus ancienne mention de pointes de flèches découvertes dans un tumulus remonte à 1638 avec la relation de la découverte d'un tumulus à Kergounadec (Cléder, Finistère) dans un courrier adressé à P. d'Hozier, juge d'armes de Louis XIII et Louis XIV. Ainsi sont décrites «deux petites pièces d'écaille faites et taillées en forme de pointes pour armer des flesches » (Chatellier, 1898b, p. 321). Entre 1880 et 1907 , de la fouille de Porz-arSaoz (Trémel, Côtes-d'Armor; Prigent, 1880) à celle du Mouden-Bras (Pleudaniel, Côtes-d'Armor; Martin et Prigent, 1907) s'est constituée la majorité du corpus des tumulus de l'âge du Bronze en Bretagne, autant pour les sépultures à pointes de flèches que pour les autres (tabl. 1). Pour le reste, il s'agit essentiellement de tombes mises au jour à l'occasion de travaux de remembrement ou de découvertes faites par des agriculteurs. Peu de sites ont fait l'objet d'une fouille extensive, ce qui fait que mis à part le volume du tertre - lorsqu'il existe -, le caveau et son mobilier, on ne connaît pas l'environnement archéologique direct de ces monuments (structures annexes, sépultures adventices).

Ces dernières années ont révélé deux sépultures à pointes de flèches de l'âge du Bronze ancien. En 1998, un exploitant agricole a découvert à Crec'h-Perros (Perros-Guirec, Côtes-d'Armor) de 25 pointes de flèches de type armoricain, d'au moins 5 poignards en alliage cuivreux, dont certains décorés de clous d'or, et de 3 haches à légers rebords du même métal. Une opération programmée, menée en octobre 2004, permit de préciser le contexte de ce dépôt funéraire. Le mobilier reposait directement sur le vieux sol et il était recouvert par un empierrement et un tertre (Blanchet, 2005). Une seconde sépulture fut mise au jour en 2006 à la suite de la construction d'un lotissement sur le site de Prat-ar-Simon-Pella en Lannilis (Finistère). Il renfermait 25 pointes de flèches, 3 poignards, dont au moins 1 décoré de clous d'or, et une hache à légers rebords en bronze (Le Goffic et Nallier, 2008).

Le corpus breton est ainsi composé de 40 tombes à pointes de flèches, dont 4 peuvent être rapportées au Campaniforme et 33 à l'âge du Bronze ancien, les 3 dernières sont insuffisamment documentées (fig. 1).

\section{Des tumulus campaniformes à pointes de flèches}

Les tumulus à pointes de flèches ont longtemps été considérés comme apparaissant à l'âge du Bronze ancien, même si certaines tombes sont reconnues comme chalcolithiques (Briard, 1984 ; Balquet, 1999). Ces dernières peuvent être attribuées au Campaniforme par leur mobilier et la similarité de leurs structures funéraires. Ces sépultures sont situées dans le sud de la Bretagne, où est concentrée la majorité des vases campaniformes de la région (fig. 1; Salanova, 2007, fig. 12).

Deux des tombes ont été fouillées par P. du Chatellier, qui a publié sommairement la sépulture de Kermenhir (Poullan-sur-Mer, Finistère; Chatellier, 1907) et plus précisément celle de Coatjou-Glas (Plonéis, Finistère; Chatellier, 1887). Toutefois les plans et les dessins du mobilier, conservés aux archives départementales du Finistère (Coativy, 2006), n'ont jusqu'ici jamais été publiés (fig. 2). Un tesson du gobelet de Kermenhir a été étudié par L. Salanova (2000, p. 211) et les deux pointes de flèches de Coatjou-Glas ont été dessinées par M. Chaigneau-Normand (1994). Le tumulus de Kermenhir est attribuable au Campaniforme par la présence d'au moins un gobelet d'allure campaniforme et deux pointes de flèches à ailerons équarris. La sépulture de Coatjou-Glas a livré tous les éléments de l'assemblage campaniforme (poignard en cuivre, pointes de flèches, brassard d'archer, perles, outils en silex et les restes d'un vase). Un des deux coffres du tumulus de Saint-Germain à Erdeven (Morbihan) peut être rattaché à cet horizon campaniforme. Il a livré des pointes de flèches et trois petites appliques en or (Gaillard, 1892; Le Rouzic, 1933; Éluère, 1982), objets trouvés fréquemment avec de la céramique campaniforme (Salanova, 2000). Le tumulus de Crubelz (Belz, Morbihan) a livré une pointe de flèche à pédoncule et ailerons équarris (Closmadeuc, 1864), armature reconnue comme typique du Campaniforme (Guyodo, 2001, p. 306; Bailly, 2002, p. 265 ; Renard 2003, p. 23 ; Furestier, 2005, p. 261). Ces quatre sépultures attribuables au Campaniforme sont toutes constituées d'un coffre en pierre, fermé par une ou plusieurs dalles de 

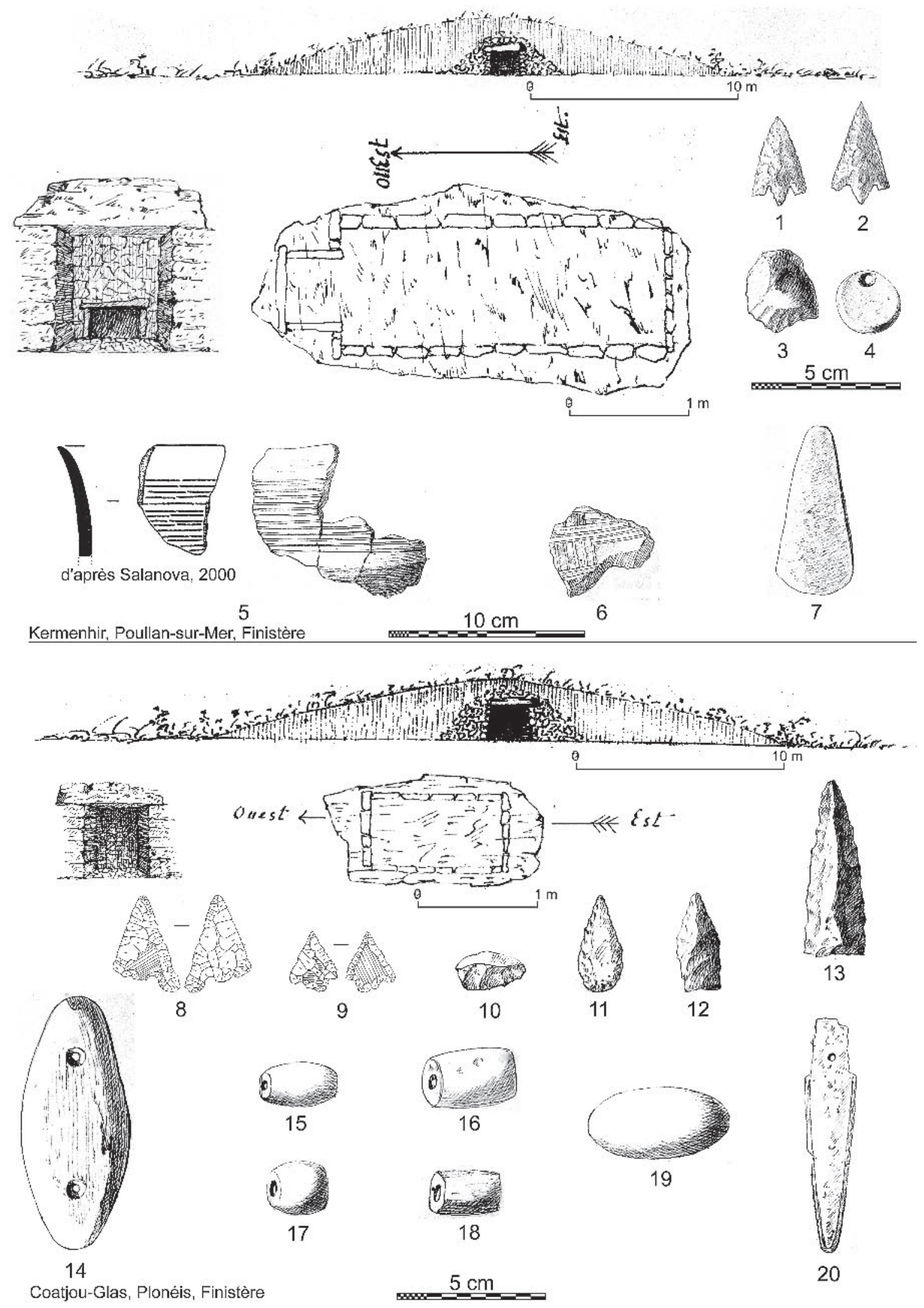

Fig. 2 - Les tumulus campaniformes de Kermenhir (Poullan-sur-Mer, Finistère) et de Coatjou-Glas (Plonéis, Finistère) : $n^{\text {os }} 1$ à 3 ) pointes de flèches et grattoir en silex; $\left.n^{\circ} 4\right)$ pendeloque en schiste; $n^{\text {os }} 5$ et 6 ) fragments de gobelet campaniforme et tesson décoré; $n^{\circ} 7$ ) hache plate en cuivre; $\mathrm{n}^{\mathrm{os}} 8$ à 13) pointes de flèches, perçoir, fragment de poignard et autres objets en silex; ${ }^{\text {os }} 14$ à 18) brassard d'archer et perles en schiste; $\left.\mathrm{n}^{\mathrm{o}} 19\right)$ galet; $\left.\mathrm{n}^{\mathrm{o}} 20\right)$ poignard en cuivre $\left(\mathrm{n}^{\mathrm{os}} 1\right.$ à 7 et 10 à 20 : archives $\mathrm{P}$. du Chatellier ${ }^{1}$ d'après clichés Y. Pailler; $\mathrm{n}^{\text {os }} 7$ et 8 d'après Chaigneau-Normand, 1994).

Fig. 2 - The Bell Beaker barrows of Kermenhir (Poullan-sur-Mer, Finistère) and Coatjou-Glas (Plonéis, Finistère): 1 to 3) flint arrowheads and scraper; 4) schist pendant; 5 and 6) part of a bell beaker and decorated sherd; 7) flat copper axe; 8 to 13) flint arrowheads, awl, fragment of dagger and others; 14 to 18) schist wristguard and beads; 19) pebble; 20) copper dagger (1 to 7 and 10 to $20:$ P. du Chatellier ${ }^{1}$ archives after photographies Y. Pailler; 7 and 8 after Chaigneau-Normand, 1994). 


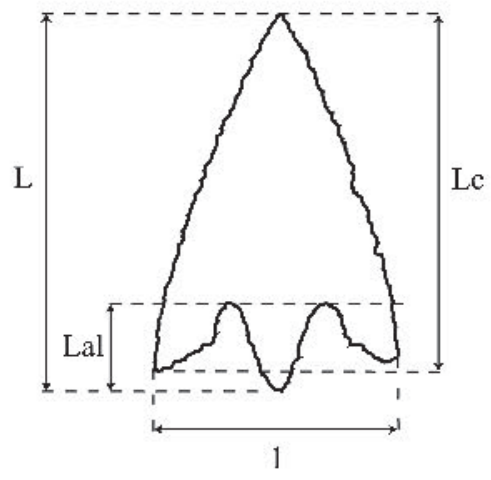

A

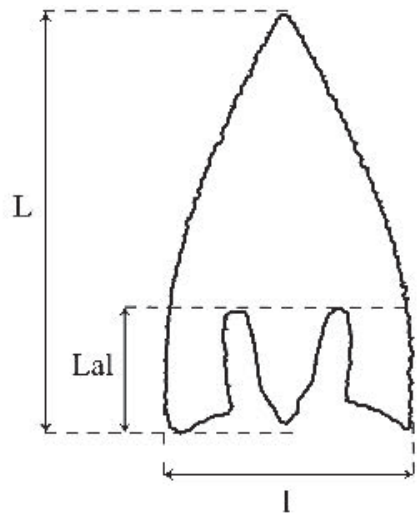

B

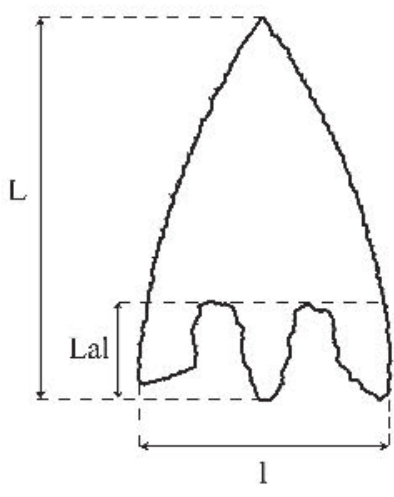

C

Schémas des prises de mesures

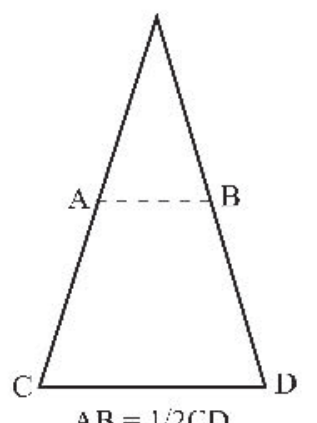

$\mathrm{AB}=1 / 2 \mathrm{CD}$

Triangulaire

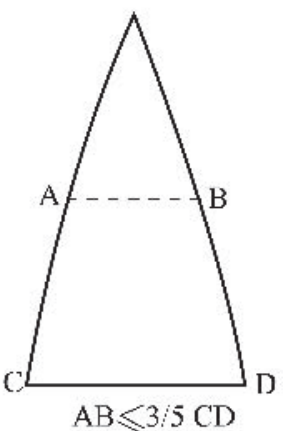

Sub-triangulaire

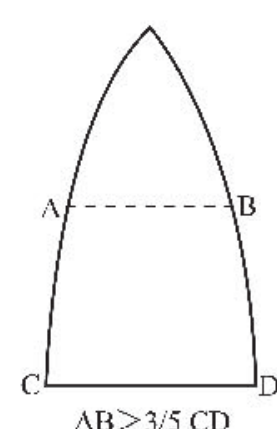

Ogival

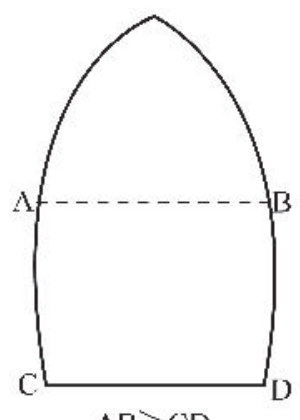

$\mathrm{AB}>\mathrm{CD}$

Ogival outrepassé

\section{Les différentes formes de pointes de flèches}

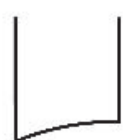

Oblique faible concave

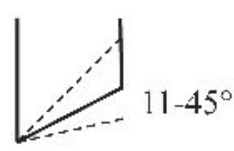

Oblique forte

Oblique faible

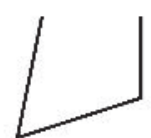

Aileron évasé

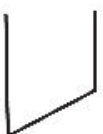

Aileron droit

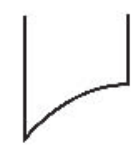

Oblique forte concave

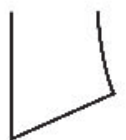

Aileton mordant

\section{Les différentes formes d'ailerons}

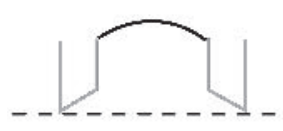

Sans pédoncule

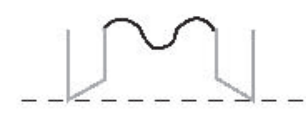

Pédoncule naissant

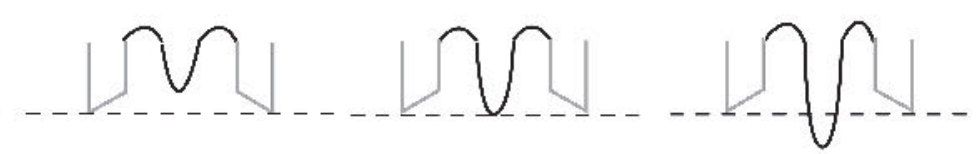

Pédoncule rentré
Pédoncule égalisé
Pédoncule dépassant

\section{Les différentes formes de pédoncules}

Fig. 3 - Schémas illustrant les mesures prises sur les pointes de flèches de type armoricain et les différentes formes de pointes de flèches, d'ailerons et de pédoncules.

Fig. 3 - Schemes of measurements on Armorican-type arrowheads and the different forms of arrowheads, barbs and tangs. 
couverture, le tout recouvert par un cairn et un tertre. Ces structures funéraires sont également caractéristiques de l'âge du Bronze ancien et en sont probablement les prémices.

\section{MÉTHODOLOGIE}

Deux critères ont servi à définir les pointes de flèches de type armoricain : les ailerons taillés en oblique et le pédoncule en «V»(Briard, 1976, p. 38). Ces deux aspects sont, de fait, sous-entendus. Toute pointe de flèche avec un pédoncule ou un aileron d'une autre forme ne pourrait être considérée comme typologiquement de type armoricain.

Les mesures ont été limitées aux données jugées pertinentes pour une construction typologique : la longueur (L), la largeur (1) et l'épaisseur (e), la longueur du corps (Lc) et la longueur des ailerons (Lal). La longueur du corps est calculée dans le cas A où le pédoncule est dépassant afin d'avoir un coefficient d'allongement comparable au cas $B$ où il ne sort pas de la forme générale. Si le pédoncule n'est pas dépassant comme dans le cas $\mathrm{C}$, la prise de mesure est équivalente à celle du cas $\mathrm{B}$. La longueur des ailerons (Lal) est mesurée dans son extension maximale (fig. 3).

Le premier problème rencontré a été celui de la typologie. Celle-ci est divisée en «ogival court», «ogival long» et «triangulaire» (Briard et Giot, 1956, p. 365). Le triangulaire se différencie clairement dans les dépôts funéraires de Keruzoret et Lambader à Plouvorn (Finistère ; Briard, 1970b) et de Cruguel (Guidel, Morbihan; Le Pontois, 1890). Dans les séries «ogival court», plusieurs pointes de flèches possèdent des bords très peu convexes. Elles se rapprochent davantage de la forme triangulaire qu'ogivale, adoptant une morphologie que l'on peut qualifier de «subtriangulaire». La difficulté de classement de certaines pièces a également été soulignée dans le cadre d'une précédente étude (Chaigneau-Normand, 1994).

Pour quantifier ces observations, nous avons mis en place un rapport entre la largeur à la moitié de la longueur du corps (AB) et la largeur à la base (CD). Lorsque $\mathrm{AB}$ équivaut à la moitié de $\mathrm{CD}$, la forme est triangulaire. Quand $\mathrm{AB}$ est inférieur ou égal à 3/5 de $\mathrm{CD}$, la forme sera considérée comme subtriangulaire. Plus AB se rapproche de la valeur de CD, plus l'armature adopte une forme ogivale. Quand $\mathrm{AB}$ est supérieur à $\mathrm{CD}$, la morphologie est dite «ogival outrepassé » (fig. 3).

Un second écueil était la limite entre les types «ogival court» et «ogival long». Afin de disposer d'une limite métrique et non d'une simple observation, nous avons calculé le rapport entre la longueur et la largeur du corps afin d'obtenir un coefficient d'allongement, facilitant les comparaisons entre des pièces qui possèdent la même forme. Une armature avec un coefficient d'allongement de 1 ne correspond pas à une pointe avec un rapport de 2,5. La distinction entre les deux nécessite le recours à l'étude de la population statistique afin de mettre en évidence des ruptures et de délimiter des classes.

Les ailerons sont qualifiés de «symétriques » lorsque la ligne formée par leurs extrémités est parallèle à l'axe transversal. Dans les autres cas, les ailerons sont dissymétriques. On peut distinguer quatre types de taille en oblique. Quand le rayon avec l'axe transversal est inférieur ou égal à 10 degrès, la taille est dite en «oblique faible». Dans les autres cas, elle est en

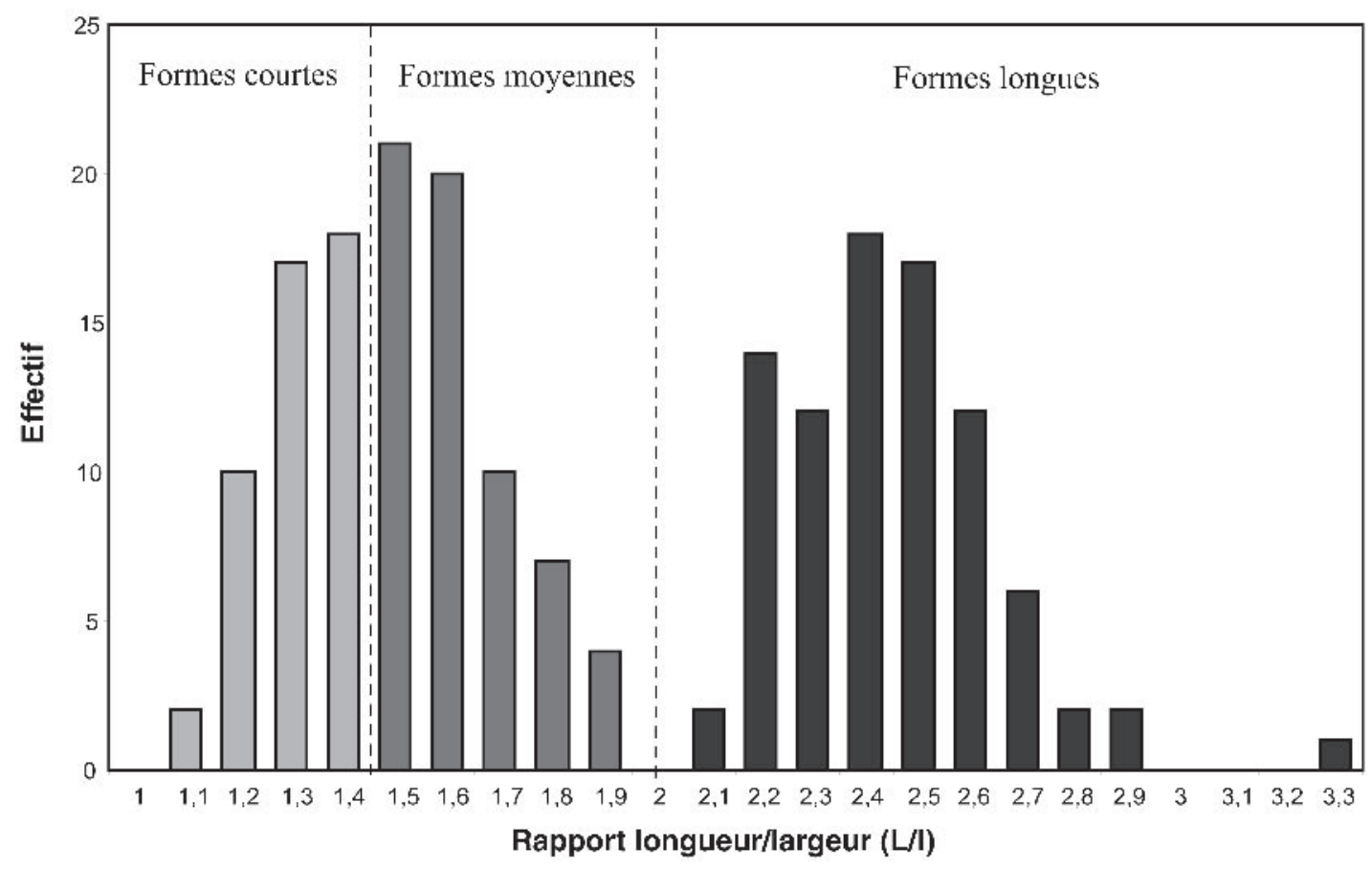

Fig. 4 - Rapport longueur/largeur mesuré sur les pointes de flèches du nord du Finistère.

Fig. 4 - Length/width ratios on arrowheads from Northern Finistère. 
oblique forte. Lorsque la taille en oblique est en arc de cercle, elle est qualifiée de «concave». La forme des ailerons peut varier suivant l'orientation de ses bords internes et externes. L'aileron est appelé «droit», si ses deux bords sont parallèles. Il est qualifié d' «évasé» quand son bord externe s'élargit. Lorsque son bord interne s'élargit, il est appelé «mordant». Le pédoncule peut être absent, naissant, rentrant, égalisé quand son extrémité proximale correspond à l'axe formé par les extrémités des ailerons et dépassant quand il déborde (fig. 3).

Les critères de description de la retouche ont été élaborés à partir du manuel Préhistoire de la pierre taillée (Inizan et al., 1995). La morphologie des enlèvements peut être parallèle ou subparallèle, leur inclinaison rasante ou semi-abrupte et leur étendue courte, envahissante ou couvrante. En raison des dimensions réduites des pointes de flèches, il n'a pas été fait de distinction entre une étendue longue ou envahissante. Sur des pièces mesurant moins de 3 centimètres de largeur, tout enlèvement qualifiable de long est envahissant. La combinaison de la retouche a été affinée d'après un article sur les techniques de taille de F. Bordes (1947). Ainsi, elle peut être transverse, en chevrons ou irrégulière.

\section{La construction typologique}

L'étude a porté sur 233 pointes de flèches recensées à partir de la bibliographie. Dans ce corpus, 106 ont pu être étudiées dans les musées et 87 à partir des dessins (mobilier perdu ou inaccessible). Cent quatrevingt-treize armatures ont donc pu être enregistrées afin d'établir une nouvelle typologie. Nous avons établi dans un premier temps une typologie descriptive. Cette typologie rend compte des formes et du style de chaque armature. Elle comporte deux éléments : la morphologie générale et les critères stylistiques du pédoncule et des ailerons.

La typologie établie dans les années 1950 distinguait des pointes ogivales courtes et longues (Briard et Giot, 1956, p. 365). Dans le nord du Finistère, cette différenciation est confirmée. Il existe une rupture franche entre des pointes de flèches qui ont un rapport L/l entre 1 et 2 et celles avec un rapport entre 2,1 et 3,3. Toutes formes confondues, il existe bien deux ensembles qui se dégagent nettement. L'étude des différentes séries a conduit à effectuer une nouvelle séparation entre les formes courtes et moyennes (fig. 4). Ainsi, dans la tombe de Kernonen (Plouvorn, Finistère), deux dépôts de pointes de flèches ont livré séparément des pointes courtes et des armatures moyennes (Briard, 1970a). L'étude des longueurs des ailerons (Lal) montre également l'existence d'ailerons courts et d'ailerons longs. La séparation se situe à 12 millimètres de longueur (fig. 5).

Le style se définit par des caractères plus discrets, qui concernent la morphologie des pédoncules, des ailerons et la taille en oblique des ailerons. L'intégration des détails stylistiques dans la construction typologique s'effectue à un niveau secondaire. Ces sous-types stylistiques se décomposent de la manière suivante : le premier chiffre pour le type de pédoncule, le deuxième pour le type de taille en oblique, le troisième pour la forme des ailerons. Nous avons précisé de manière indicative la symétrie ou la dissymétrie des deux ailerons (fig. 6).

L'étude morphologique a mis en évidence huit types suivant les critères de la forme, du rapport L/l et de la

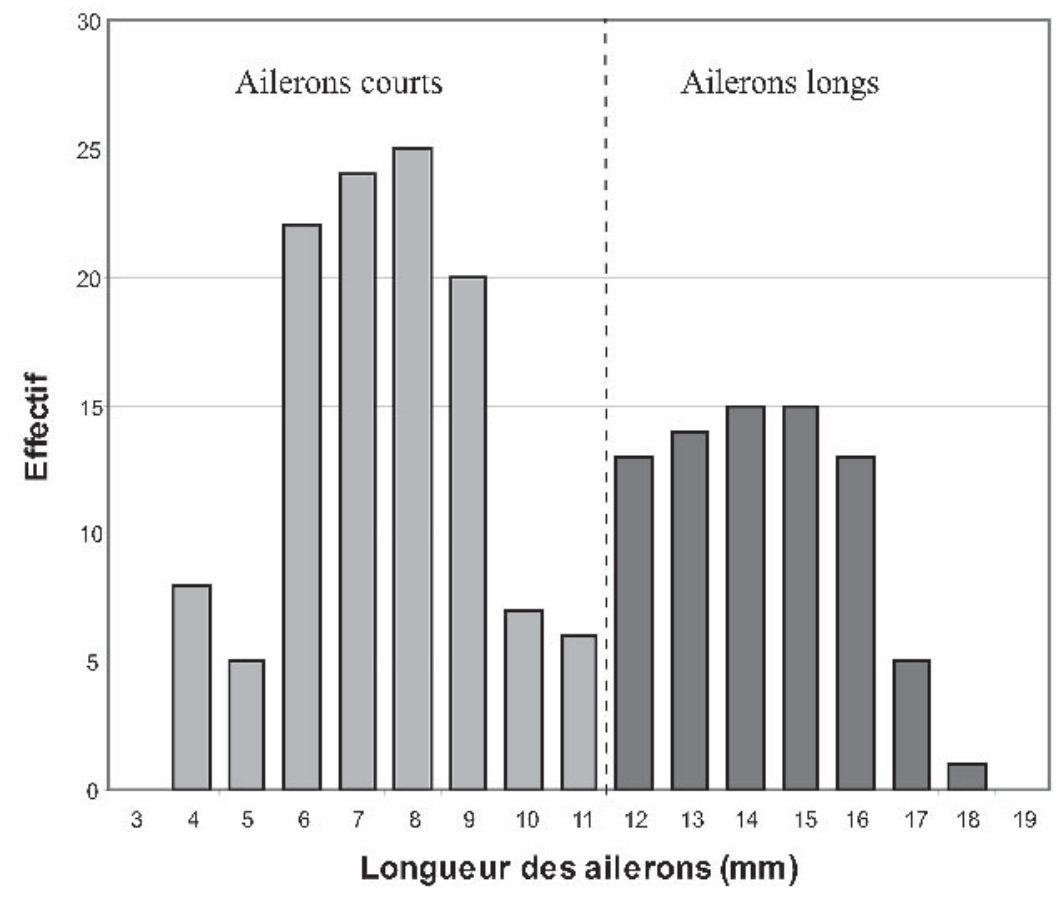

Fig. 5 - Longueur des ailerons sur les pointes de flèches du nord du Finistère.

Fig. 5 - Barbs length on arrowheads from Northern Finistère. 


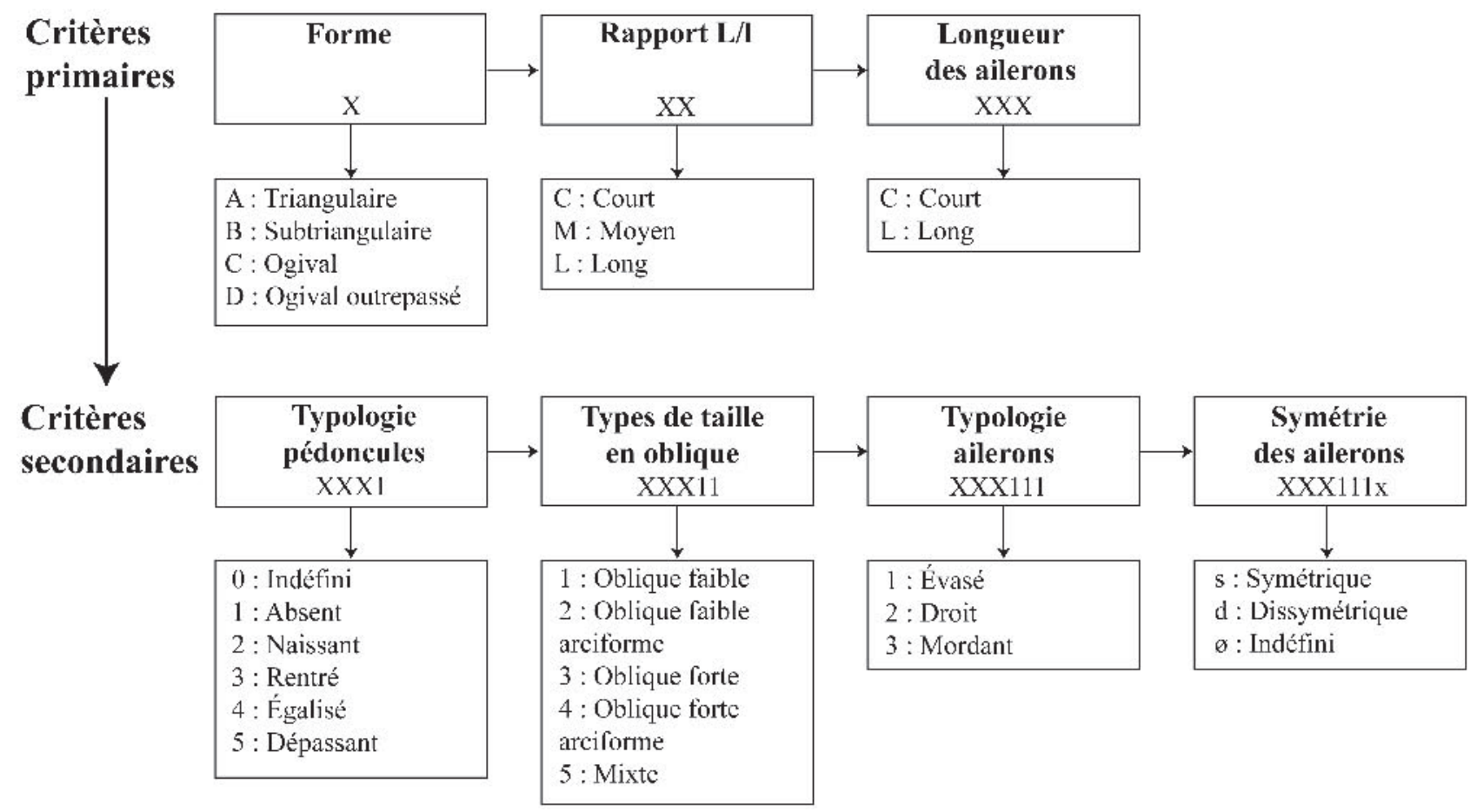

Fig. 6 - Schéma de la construction typologique. Fig. 6-Scheme of the typological construction.

\begin{tabular}{|l|c|c|c|}
\hline \multicolumn{1}{|c|}{ Type } & Forme & $\begin{array}{c}\text { Rapport } \\
\text { L/I }\end{array}$ & $\begin{array}{c}\text { Longueur } \\
\text { des } \\
\text { ailerons }\end{array}$ \\
\hline Cazin & Subtriangulaire & $1-2$ & Court \\
\hline Kerguévarec & Ogivale & $1-1,49$ & Court \\
\hline Kernonen & Ogivale & $1,5-1,99$ & Court \\
\hline Kervini & Ogivale & $2-3$ & Court \\
\hline Limbabu & Ogivale & $2-3,5$ & Long \\
\hline Graeoc & Ogivale outrepassée & $2-3$ & Long \\
\hline Cruguel & Triangulaire & $1,5-3$ & $\begin{array}{c}\text { Court } \\
\text { à long }\end{array}$ \\
\hline Keruzoret & $\begin{array}{c}\text { Triangulaire } \\
\text { sans pédoncule }\end{array}$ & $2-3$ & $\begin{array}{c}\text { Court } \\
\text { à long }\end{array}$ \\
\hline
\end{tabular}

Tabl. 2 - Les types de pointes de flèches et leurs caractéristiques. Table 2 - Types of arrowheads and theirs characteristics.

longueur des ailerons. Afin de simplifier leur appellation, nous leur donnons le nom du site où le type apparaît comme le plus représentatif et le plus abondant (tabl. 2).

Le travail a permis de donner une limite entre des pointes ogivales courtes, ogivales moyennes et ogivales longues, avec les types Kerguévarec (fig. 7, $\mathrm{n}^{\text {os }} 1,3,8$, 9,13 à $15,17,19$ à 22, 27 à 29, 32 et 33), Kernonen (fig. 7, nos 4 à 7, 10 à 12, 16, 18, 23 à 25 et 30), Kervini et Limbabu (fig. 8, $\mathrm{n}^{\text {os }} 3$ à 16). Les ogivales longues ont été séparées entre celles qui possédaient des ailerons courts (type Kervini) ou longs (type Limbabu). Les triangulaires ont été divisées suivant la présence (type Cruguel; fig. 9, $\mathrm{n}^{\text {os }} 1$ et 2 ) ou l'absence d'un pédoncule (type Keruzoret; fig. 9, n ${ }^{\text {os }} 3$ à 10). L'étude a distingué deux nouvelles formes, qui sont le «subtriangulaire», qui se singularise par des bords peu convexes (type Cazin; fig. 7, $\mathrm{n}^{\text {os }} 2,26$ et 31 ), et «l'ogival outrepassé » (type Graeoc; fig. $8, \mathrm{n}^{\text {os }} 1$ et 2 ), version fusiforme du type Limbabu (fig. $8, \mathrm{n}^{\text {os }} 3$ à 16). Notre typologie confirme les principaux types mis en évidence en 1900 par A. Martin (1900, fig. 2).

\section{LES POINTES DE FLÈCHES DE TYPE ARMORICAIN DU NORD DU FINISTÈRE}

\section{Les matières premières}

Les pointes de flèches de type armoricain du nord du Finistère ont toutes été taillées dans du silex. Plusieurs matières premières ont été identifiées, mais l'essentiel du corpus a été réalisé dans un silex blond translucide à grain fin. D'autres silex translucides ont une teinte gris clair, rouge, marron ou noir. Des silex opaques ont un grain plus grossier et adoptent des teintes gris clair à gris foncé. Dans le nord du Finistère, il y a une opposition franche entre les pointes de formes courtes ou moyennes (types Cazin, Kerguévarec, Kernonen; fig. 7) qui sont réalisées dans des matières premières de teintes variées et les pointes de flèches ogivales longues et triangulaires (types Kervini, Limbabu, Graeoc, Keruzoret, Lambader; fig. 8 et 9) façonnées dans un silex blond translucide (tabl. 3).

Le silex blond translucide possède un grain fin. Il contient fréquemment de fines inclusions calcaires atteignant régulièrement 4 à 5 millimètres de longueur. 

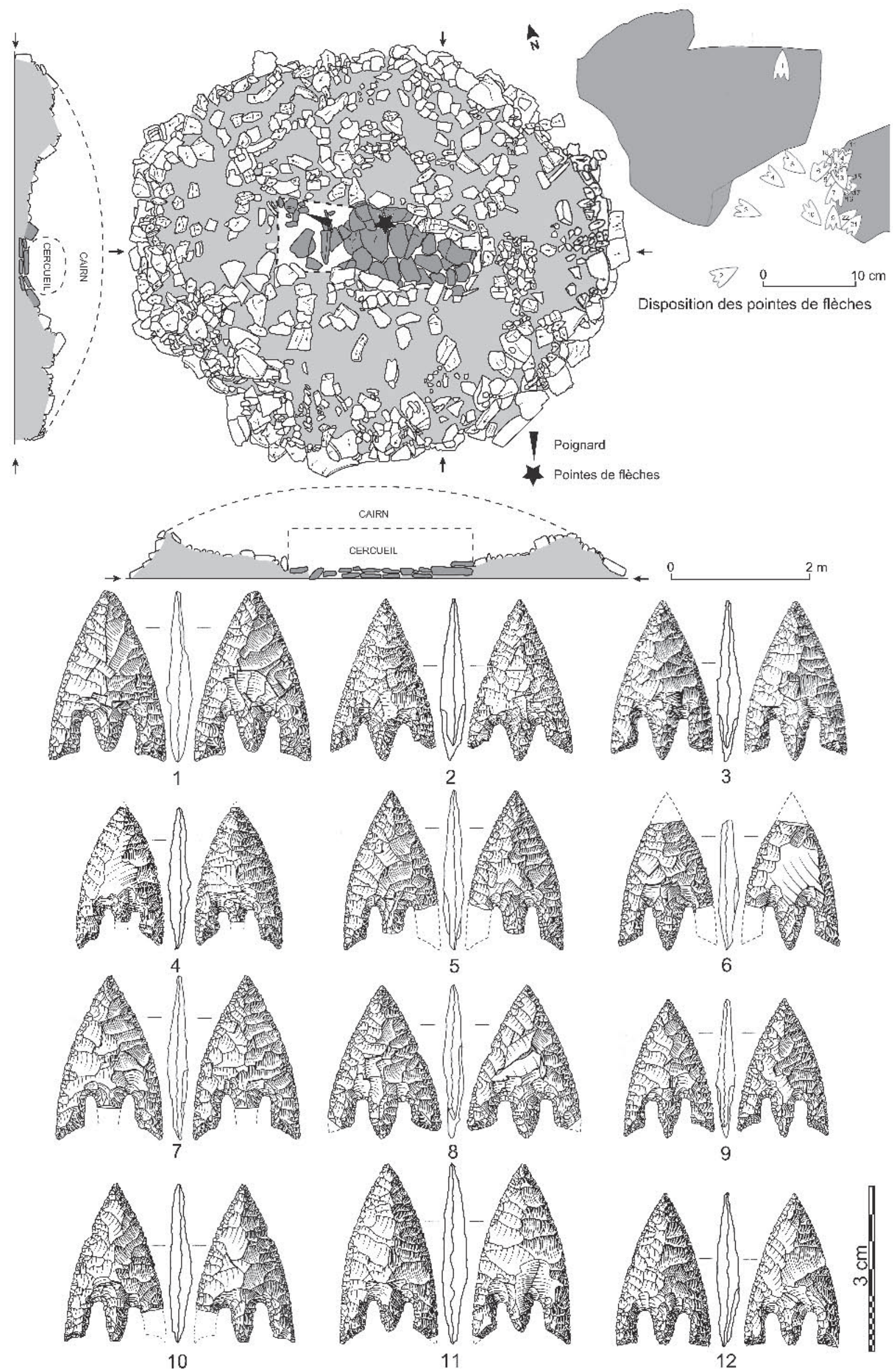

9

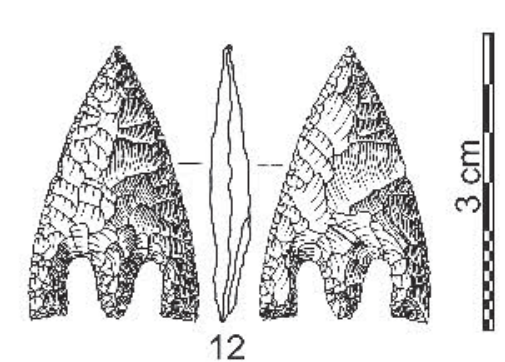



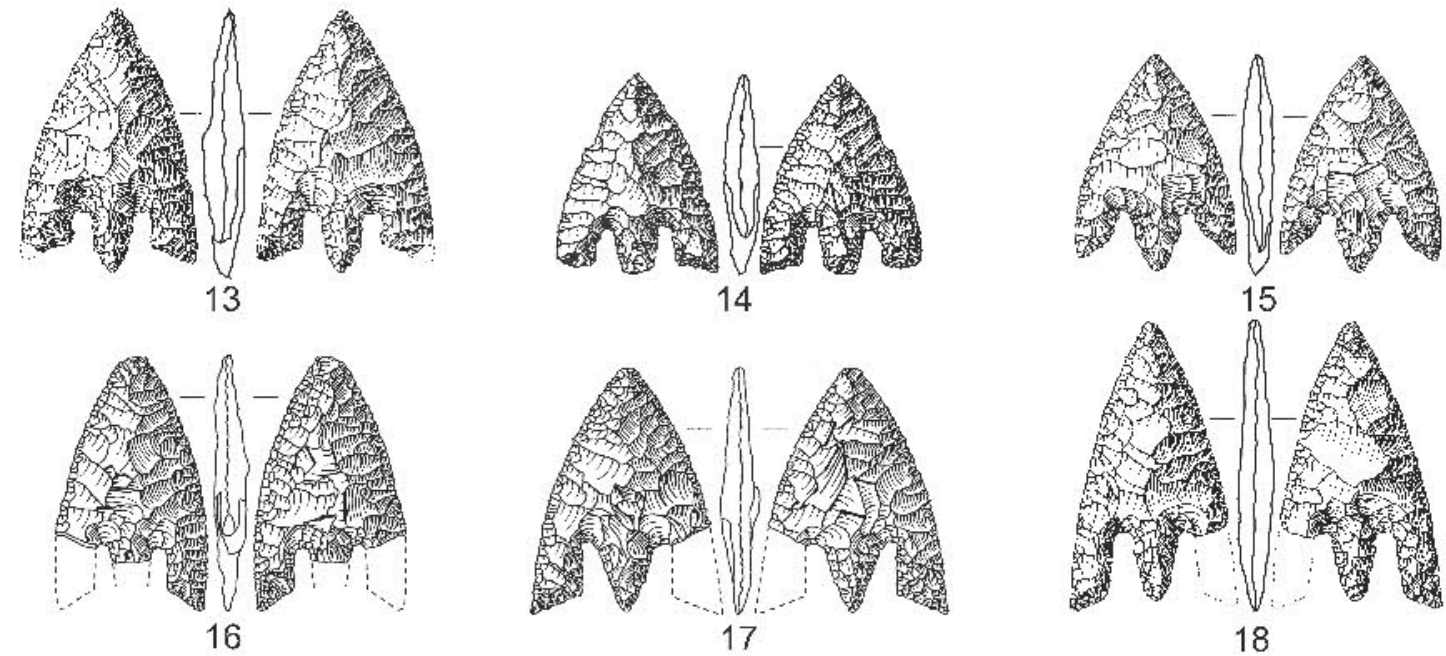

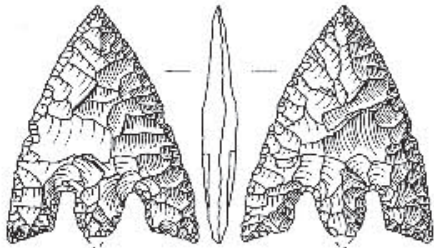

19

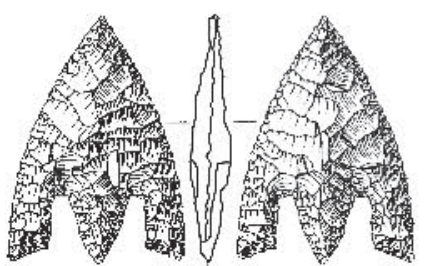

22

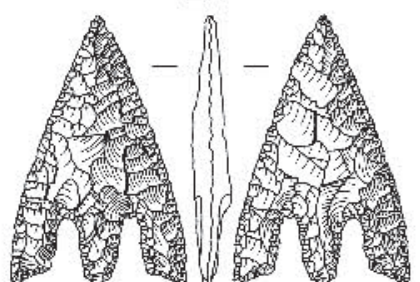

25

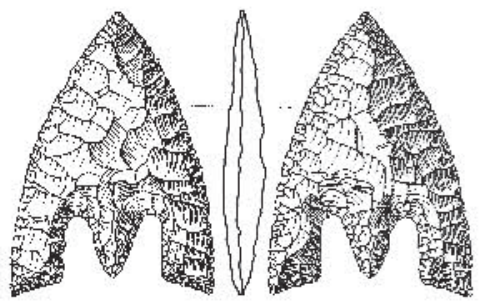

28

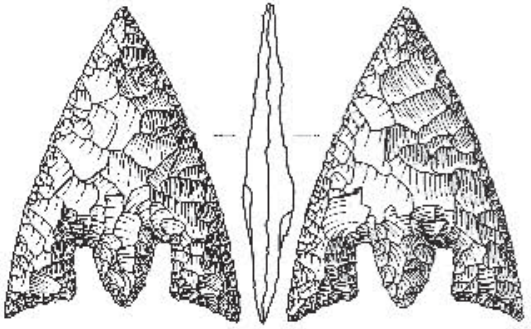

31

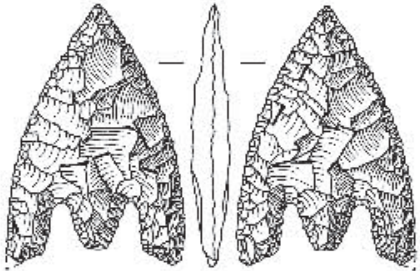

20

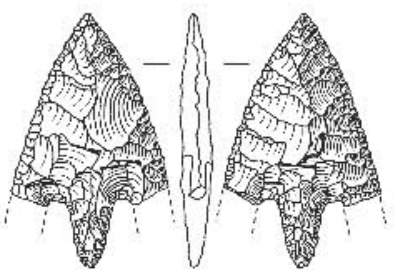

23

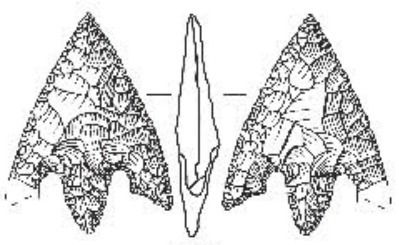

26

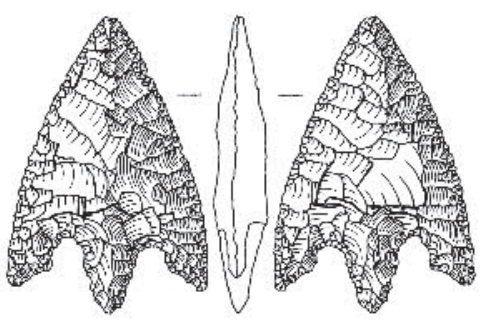

29

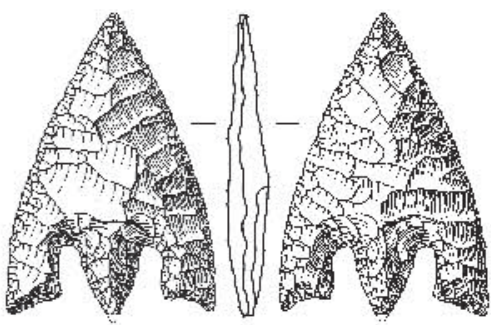

32

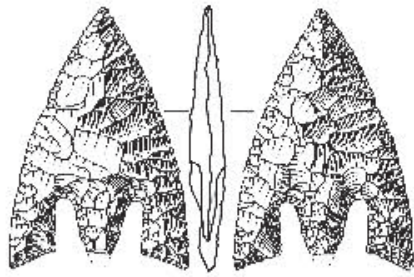

21

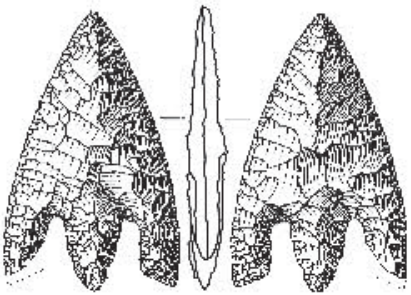

24

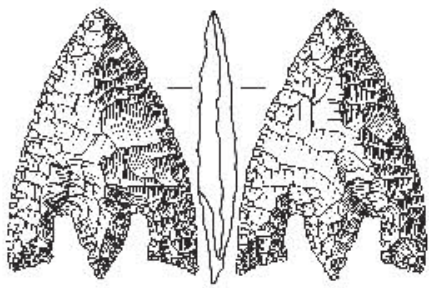

27

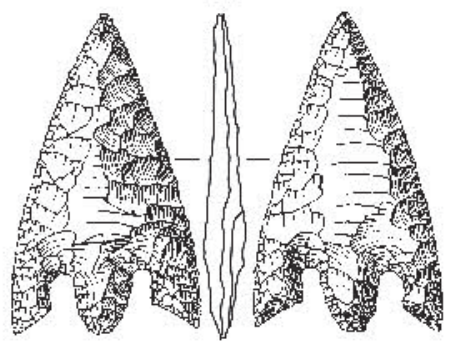

30

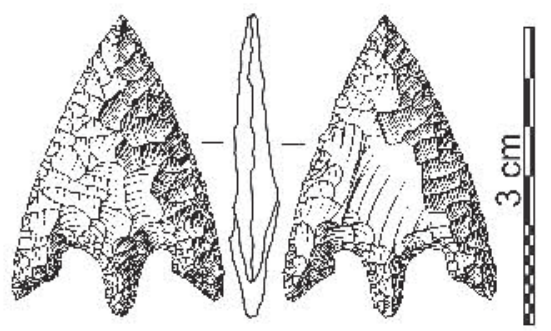

33

Fig. 7 (gauche et ci-dessus) - Plan et mobilier du tumulus de Cazin, Plouigneau, Finistère (plan d'après Briard, 1977a; dessins C. Nicolas)

Fig. 7 - Plan and goods from Cazin barrow, Plouigneau, Finistère (plan after Briard, 1977a; drawings C. Nicolas). 

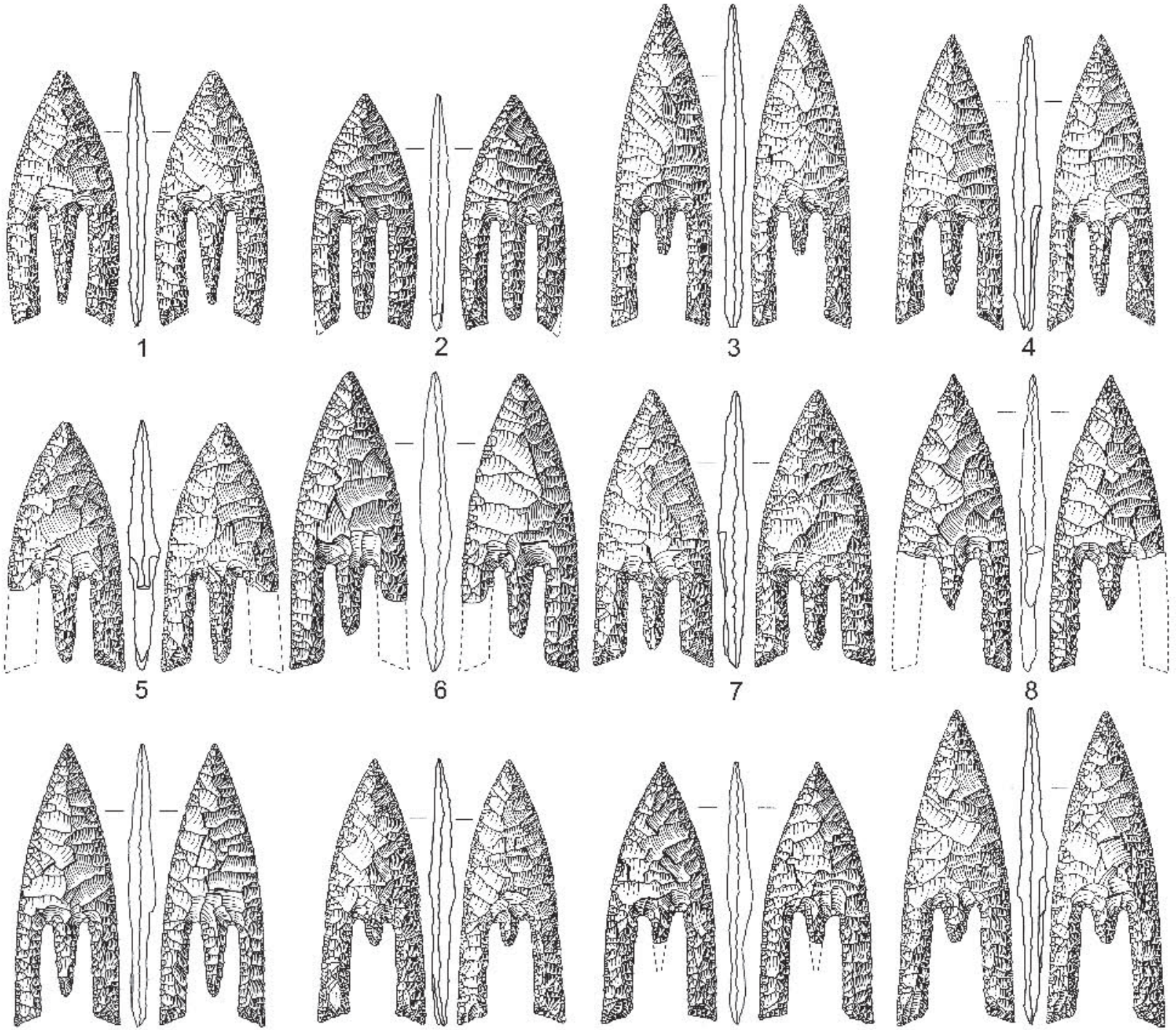

9

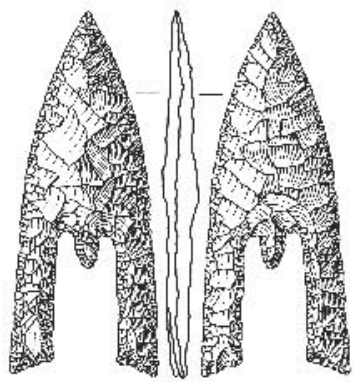

10
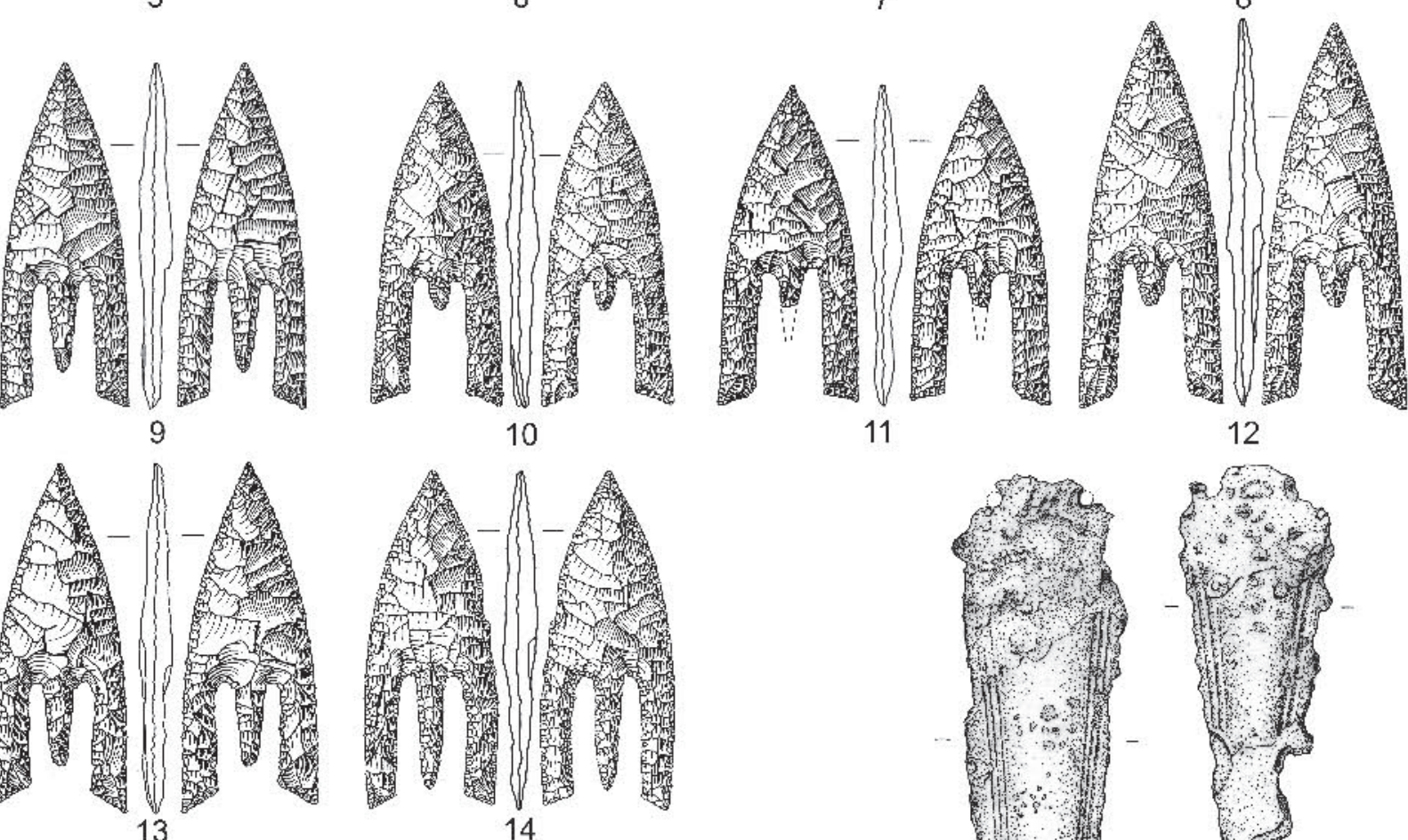

11

12

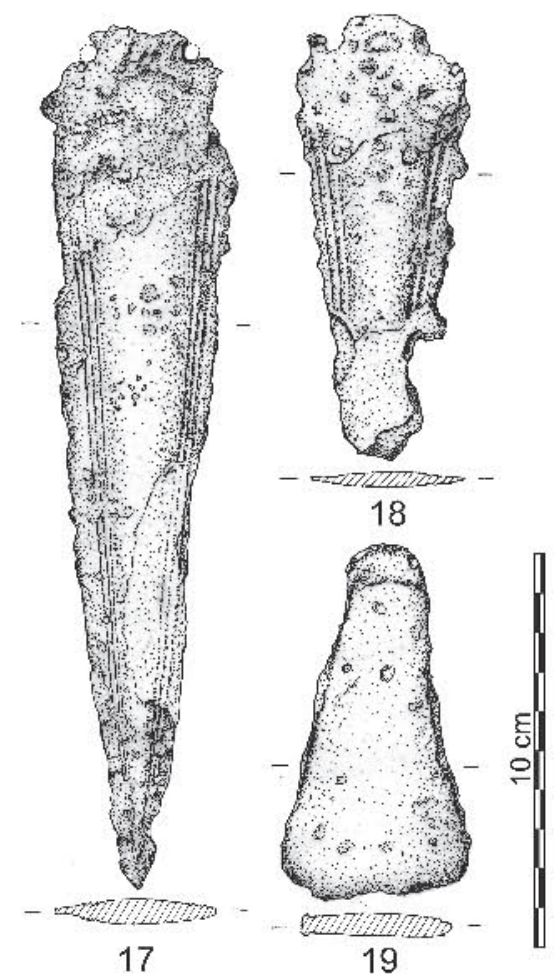

Fig. 8 - Mobilier de la sépulture de Graeoc 2, Saint-Vougay, Finistère (dessins C. Nicolas).

Fig. 8 - Goods from Graeoc 2 grave, Saint-Vougay, Finistère (drawings C. Nicolas). 

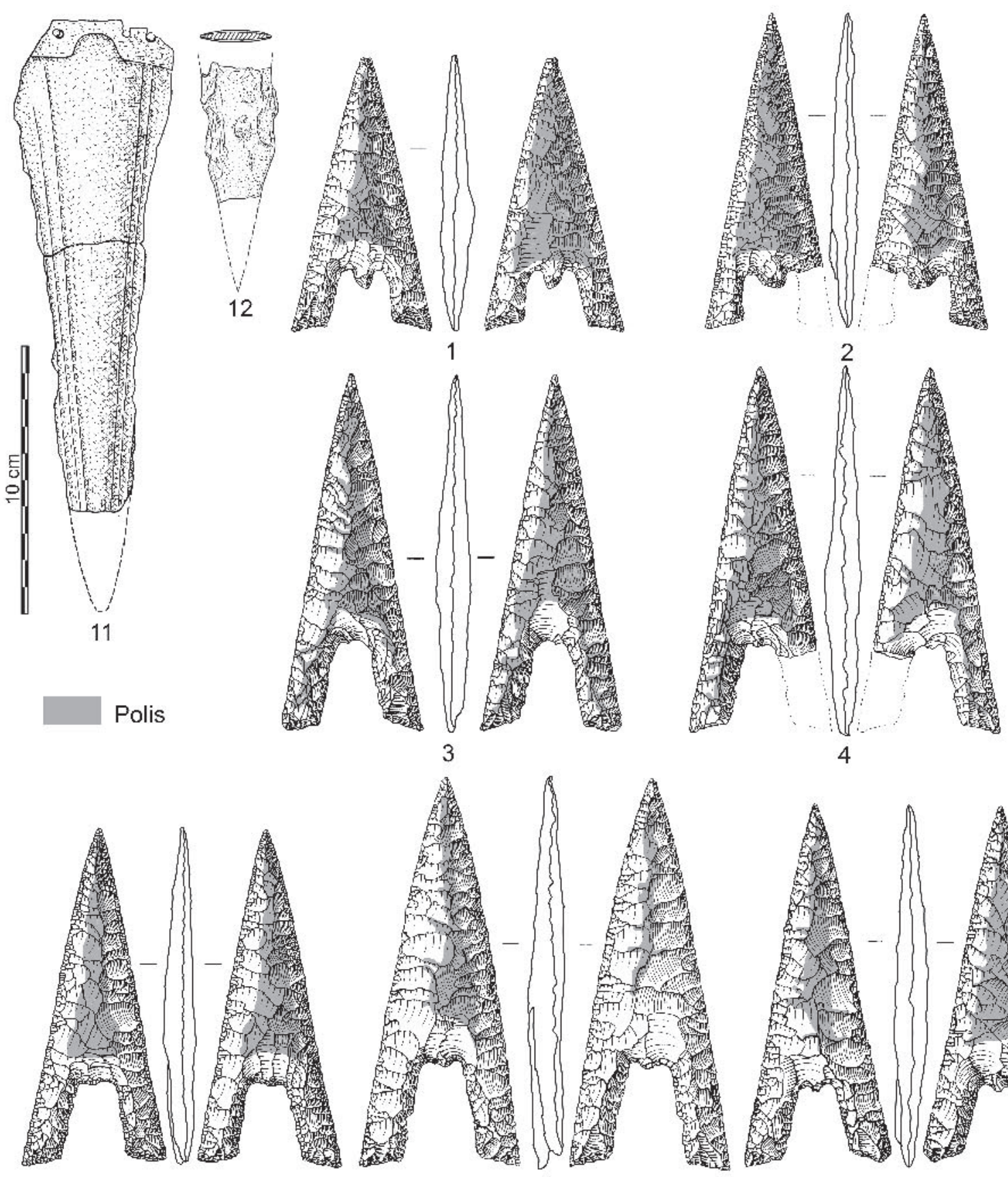

5

6
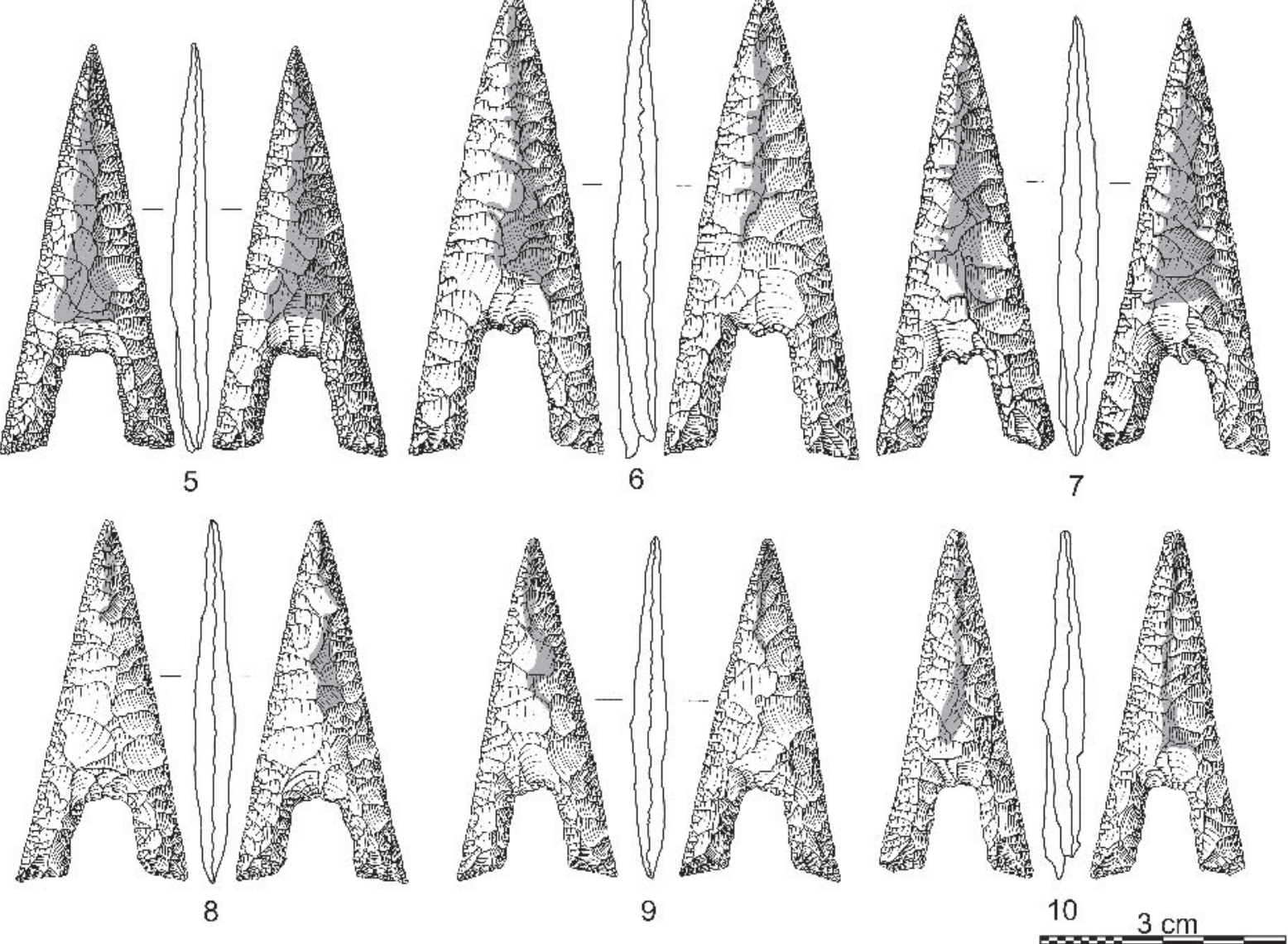

Fig. 9 - Mobilier du tumulus de Keruzoret, Plouvorn, Finistère ( $\mathrm{n}^{\text {os }} 1$ à 10 : dessins C. Nicolas; ${ }^{\text {os }} 11$ et 12 d'après Briard, $\left.1970 b\right)$

Fig. 9 - Goods from Keruzoret barrow, Plouvorn, Finistère (1 to 10: drawings C. Nicolas; 11 and 12 after Briard, 1970b). 


\begin{tabular}{|l|c|c|c|}
\hline \multicolumn{1}{|c|}{ Type } & $\begin{array}{c}\text { Nombre } \\
\text { de pointes } \\
\text { de flèches }\end{array}$ & $\begin{array}{c}\text { Nombre de faciès } \\
\text { de matières } \\
\text { premières }\end{array}$ & $\begin{array}{c}\text { Part du silex } \\
\text { blond } \\
\text { translucide }\end{array}$ \\
\hline Cazin & 3 & 3 & 0 \\
\hline Kerguévarec & 22 & 5 & $7(31,8 \%)$ \\
\hline Kernonen & 22 & 4 & $8(36,4 \%)$ \\
\hline Kervini & 2 & 1 & 2 \\
\hline Limbabu & 47 & 1 & $47(\mathbf{1 0 0} \%)$ \\
\hline Graeoc & 2 & 1 & 2 \\
\hline Cruguel & 2 & 1 & 2 \\
\hline Keruzoret & 8 & 1 & $8(\mathbf{1 0 0} \%)$ \\
\hline
\end{tabular}

Tabl. 3 - Types de pointes de flèches et variété des matières premières. Table 3 - Types of arrowheads and variety of raw materials.

Des inclusions de 6 à 10 millimètres ont pu gêner la taille. Il existe également des exemplaires faiblement teintés et contenant très peu d'inclusions. À deux occasions, il a été rencontré des inclusions particulières, pouvant aider à déterminer ce silex : des bryozoaires coloniaux et une microgéode de quartz.

Le Massif armoricain peut difficilement avoir fourni ce silex blond translucide à grain fin. Certains galets provenant des cordons littoraux ont des teintes semblables, comme sur le site d'habitat Campaniforme-Bronze ancien de Beg-ar-Loued (île Molène, Finistère; Pailler et al., 2010), mais la texture du silex est plus grossière. Ces galets roulés sont souvent microfracturés, ce qui représente une gêne pour débiter et retoucher les supports de pointes de flèches de type armoricain. Aucune des pointes de flèches ne présente de cortex ou de zones sous-corticales qui appuieraient l'hypothèse d'une origine locale de ce silex.

Il faut se tourner vers les gisements de silex extérieurs au Massif armoricain. Les marges orientales de ce massif se trouvent à environ 300 kilomètres du nord du Finistère. Les meilleurs échantillons de comparaison ont été obtenus avec des silex translucides du Crétacé. On retrouve des silex blonds translucides accompagnés de variantes marron dans la basse vallée de la Seine (E. Ghesquière, com. pers.). Le silex de Meusnes, provenant de la vallée du Cher, présente, en plus d'un silex blond, une grande variété de teintes (J. Pelegrin, com. pers.).

Une comparaison effectuée avec un échantillon de silex du Turonien inférieur provenant de Selles-surCher (Loir-et-Cher) a montré des résultats probants. On retrouve sur ce silex des bryozoaires coloniaux (P. Forré, com. pers.) et des microgéodes de quartz. D'ailleurs, des blocs de silex du Turonien inférieur sont présents dans les alluvions du Cher, ils sont ensuite transportés par la Loire jusque dans l'estuaire à SaintNazaire (Loire-Atlantique). De là, les courants marins ont disséminé des petits nodules le long des côtes atlantiques. P. Forré (com. pers.) observe ainsi des rognons d'une taille maximale de 10 centimètres sur les cordons littoraux du Morbihan. Toutefois, ces petits rognons de silex, microfracturés et charriés par les courants marins, ont difficilement pu être exploités pour réaliser les pointes de flèches de type armoricain. Enfin, les silex de la frange ouest du Bassin parisien sont encore largement méconnus, et on ne peut pas exclure d'autres sources d'approvisionnement en silex blond (J. Pelegrin, com. pers.).

Les tailleurs de pointes de flèches de type armoricain ont eu besoin d'avoir accès à un silex de très bonne qualité. C'est dans ce silex blond qu'ils ont trouvé une matière première avec une aptitude à la taille qui leur convenait. L'utilisation systématique de ce silex dans sa teinte blonde (tabl. 3) laisse à penser que cette matière première devait également répondre à des critères esthétiques. Cette couleur blonde n'est pas sans rappeler le jaune de l'or, du bronze ou de l'ambre que l'on retrouve associés dans les dépôts funéraires, comme sur le site de Kernonen (Plouvorn, Finistère; Briard, 1970a).

L'accès à ce silex blond translucide devait être limité. Sans cela, il est difficile d'expliquer la présence d'inclusions calcaires importantes qui ont gêné la taille et empêché le plein développement de la forme de la pointe de flèche. Si le tailleur de l'âge du Bronze ancien avait disposé d'une quantité suffisante de matière première, il aurait écarté un support contenant une inclusion de 10 millimètres gênant la taille. Sur une pointe de flèche de Coatanéa (Bourg-Blanc, Finistère), une telle inclusion a clairement empêché le développement du pédoncule. Le projet de réaliser une pointe de flèche de 3 millimètres d'épaisseur dans un support présentant une inclusion importante et par la suite de déposer l'objet dans une sépulture sous-entend que ce silex blond était un bien rare et précieux.

\section{Types et répartition}

La répartition des différents types de pointes de flèches montre des situations relativement diverses. On observe une répartition diffuse des types Cazin, Kerguévarec et Kernonen (fig. 10). Les types Graeoc, Cruguel et Keruzoret se trouvent en quantité trop faible pour en tirer des conclusions (fig. 10). Une très forte concentration du type Limbabu (70,5\%) se reconnaît dans le Léon avec des présences ponctuelles dans les autres parties de la Basse-Bretagne (fig. 10). Ce sont également les armatures qui ont sans doute demandé le plus haut niveau de savoir-faire afin de dégager des ailerons longs. Toutes les séries de type Limbabu sont réalisées dans le silex blond translucide. La répartition spatiale, le savoir-faire technique et l'homogénéité de la matière première indiquent que ces armatures sont une production propre au Léon. Des pointes de flèches de type Kervini leur sont ponctuellement associées. Hors du Léon, on peut distinguer deux sortes de dépôts, ceux assez proches de ceux du Léon dans lesquels le type Limbabu est majoritaire (la Motta, Cosmaner; fig. 11, $\mathrm{n}^{\text {os }} 4$ et 7) et d'autres plus éloignés dans lesquels il est en minorité (TossenKergourognon, Tossen-Rugouec, Kervini, Coët-erGaff; fig. 11, $\mathrm{n}^{\text {os }}$ 5, 6, 8 et 9). La circulation de pointes de type Limbabu peut s'expliquer de plusieurs manières : des imitations locales ou des importations de pointes de flèches de type Limbabu lorsque la série est homogène. 


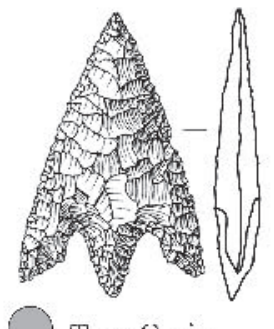

Type Cazin
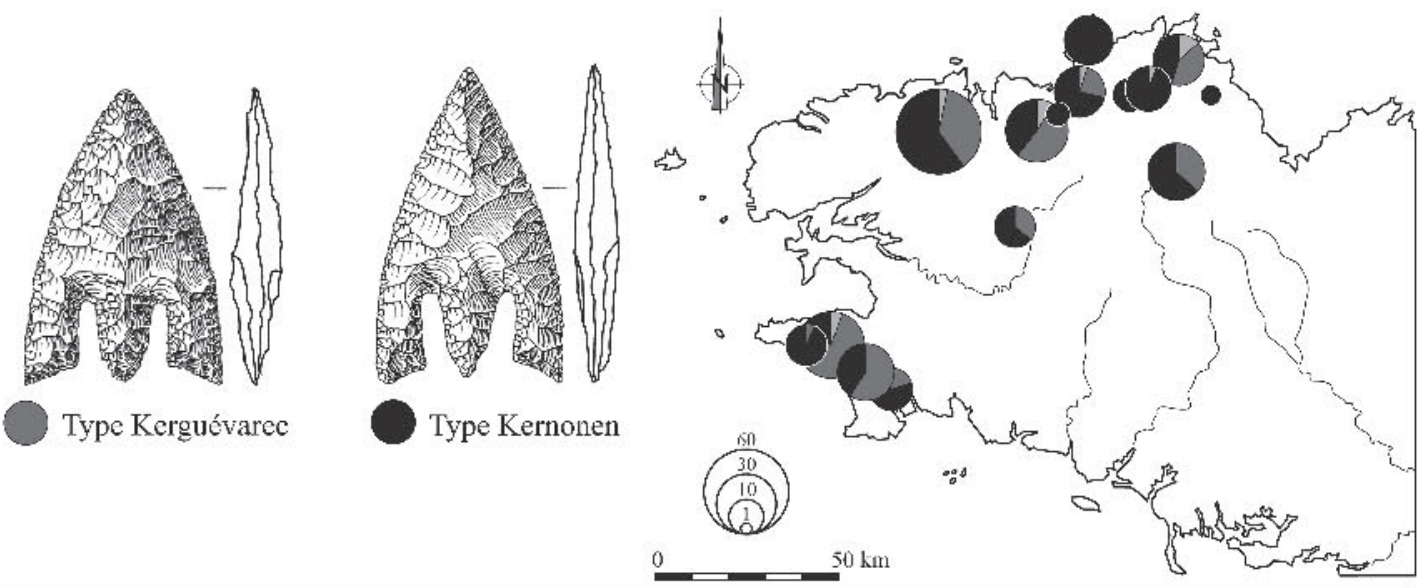

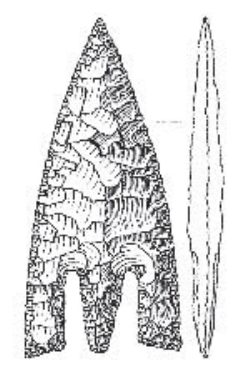

Type Kervini

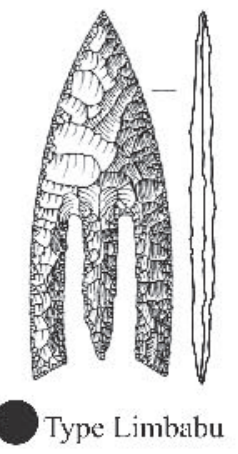

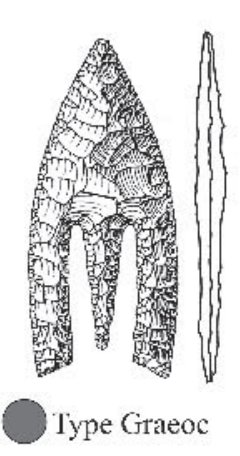

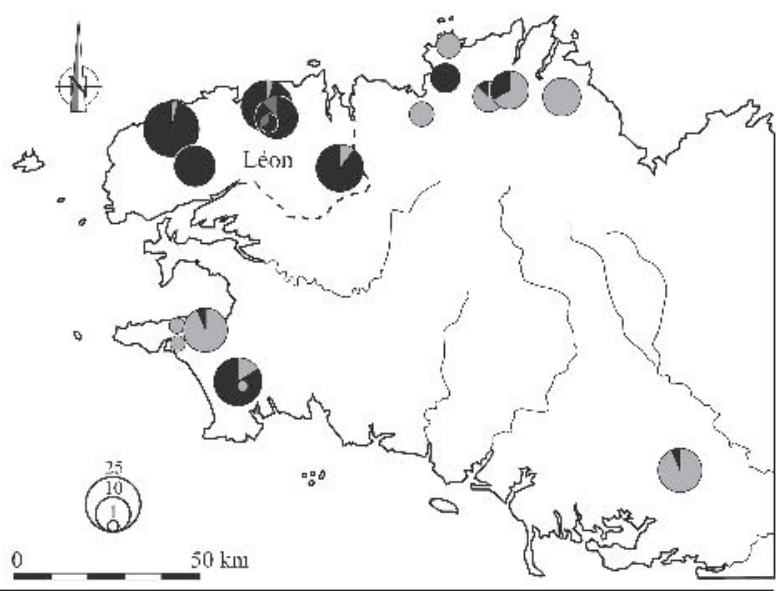

$0 \quad-\quad-50 \mathrm{~km}$
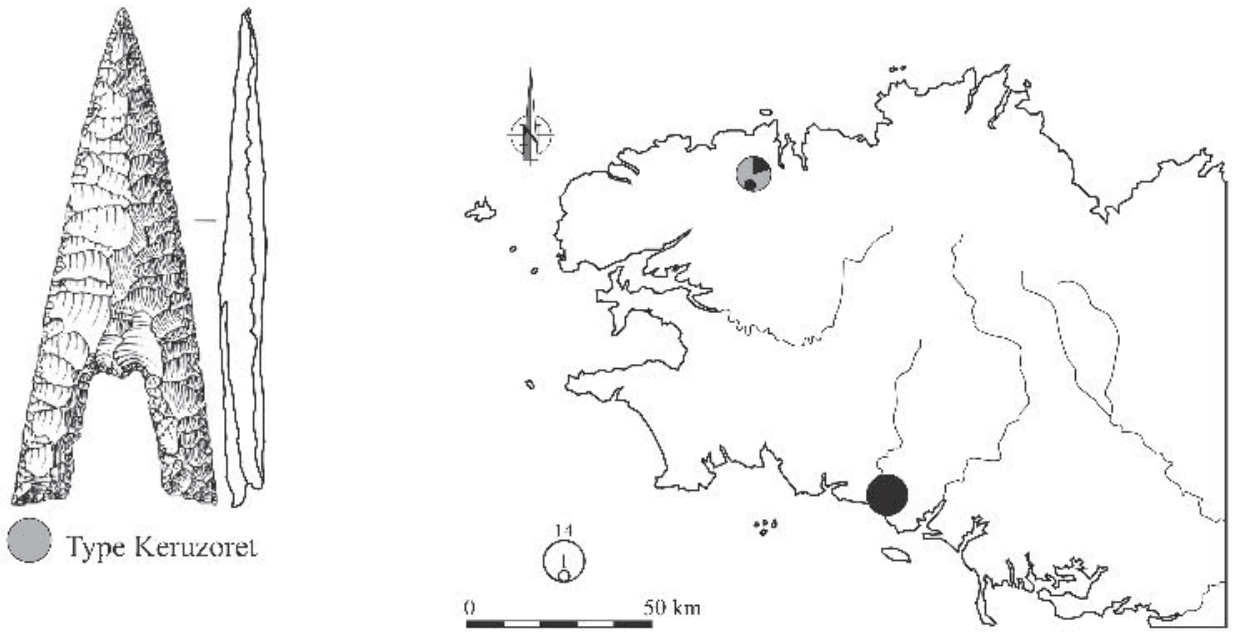

Fig. 10 - Types et répartition des pointes de flèches armoricaines.

Fig. 10 - Types and distribution of Armorican arrowheads.

Ces deux modèles, imitation ou diffusion, ont pu coexister. Les armatures provenant des tumulus de la Motta et de Cosmaner (fig. 11, $\mathrm{n}^{\text {os }} 4$ et 7) offrent de bonnes correspondances avec les exemplaires du Léon (fig. $11, \mathrm{n}^{\text {os }} 1$ à 3 ). Les deux pointes de Kervini (fig. 11, $\mathrm{n}^{\circ}$ 8) et Coët-er-Gaff (fig. 11, $\mathrm{n}^{\circ}$ 9) possèdent un corps développé. Dans les deux cas, une unique pointe de type Limbabu est accompagnée d'armatures de type Kervini et pourrait correspondre à une importation ou à une très bonne imitation locale. Les pointes de flèches de type Limbabu des sites de TossenKergourognon et de Tossen-Rugouec (Prat, Côtes- 


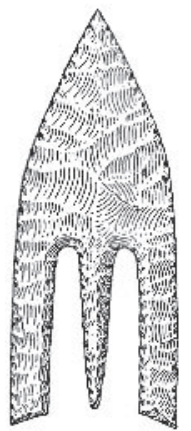

1

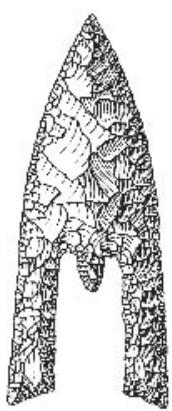

2

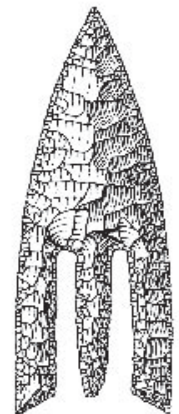

3

Pays de Léon (nord du Finistère)

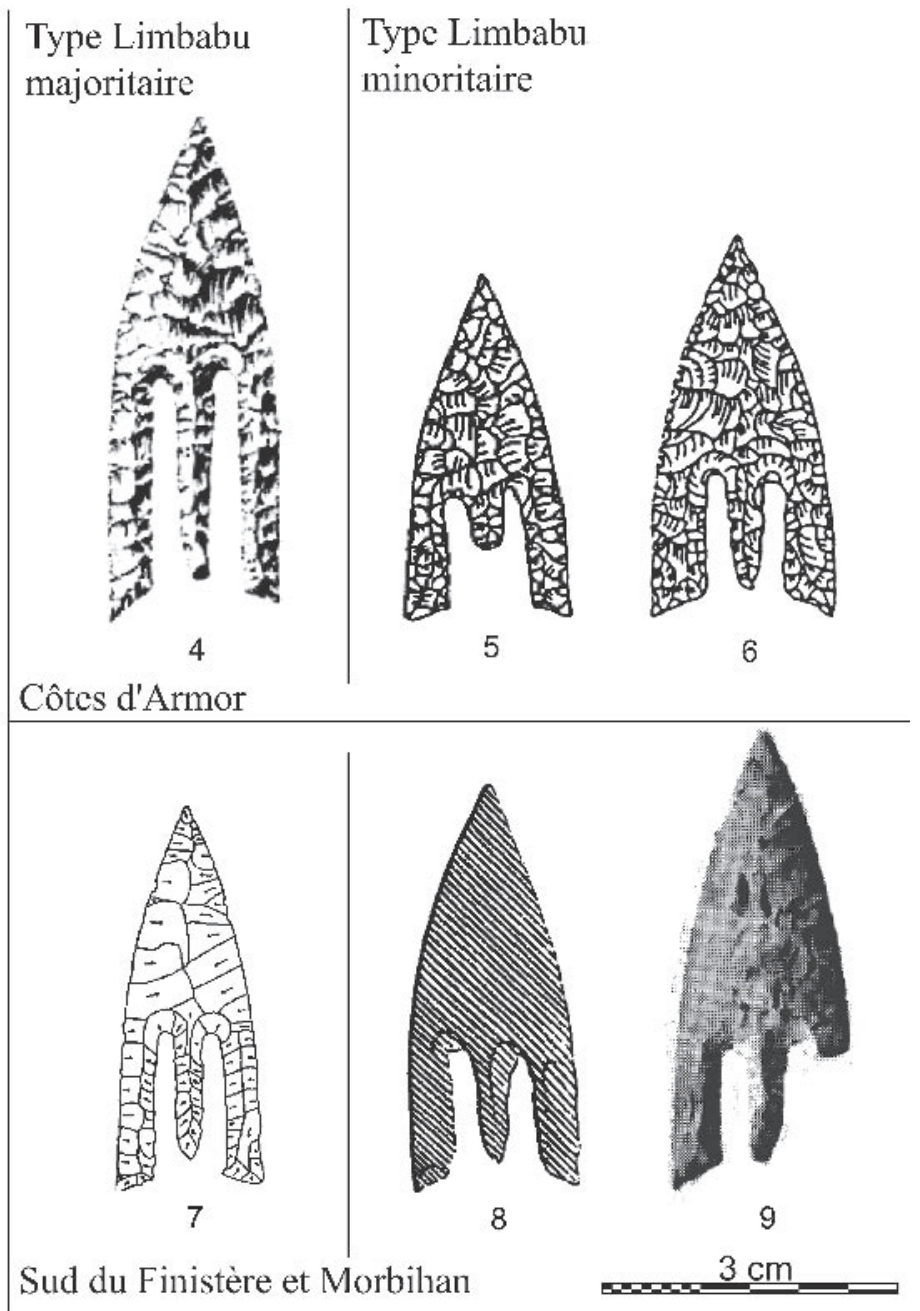

Fig. 11 - Comparaison entre les pointes de flèches de type Limbabu du Léon et du reste de la Bretagne : no 1) Goarillac'h, PlounévezLochrist, Finistère (d'après Mortillet et Mortillet, 1881); $\mathrm{n}^{\circ}$ 2) Graeoc 2, Saint-Vougay, Finistère (dessin C. Nicolas); $\left.\mathrm{n}^{\circ} 3\right)$ Limbabu, Saint-Thégonnec, Finistère (dessin C. Nicolas); $n^{\circ}$ 4) la Motta, Lannion, Côtes-d'Armor (d'après Butler et Waterbolt, 1974); ${ }^{\circ}$ 5) Tossen-Kergourognon, Prat, Côtes-d'Armor (d'après Balquet, 2001); nº 6) Tossen-Rugouec, Prat, Côtes-d'Armor (d'après Balquet, 2001); $\mathrm{n}^{\circ}$ 7) Cosmaner, Plonéour-Lanvern, Finistère (d'après Chaigneau-Normand, 1994); ${ }^{\circ}$ 8) Kervini, Poullan-sur-Mer, Finistère (d'après Halna du Fretay, 1888); n 9) Coët-er-Gaff, Elven, Morbihan (d'après Marsille, 1913).

Fig. 11 - Comparison between arrowheads of the Limbabu type from Léon and from the rest of Brittany: 1) Goarillac'h, PlounévezLochrist, Finistère (after Mortillet and Mortillet, 1881); 2) Graeoc 2, Saint-Vougay, Finistère (drawing C. Nicolas); 3) Limbabu, Saint-Thégonnec, Finistère (drawing C. Nicolas); 4) La Motta, Lannion, Côtes-d'Armor (after Butler and Waterbolt, 1974); 5) TossenKergourognon, Prat, Côtes-d'Armor (after Balquet, 2001); 6) Tossen-Rugouec, Prat, Côtes-d'Armor (after Balquet, 2001); 7) Cosmaner, Plonéour-Lanvern, Finistère (after Chaigneau-Normand, 1994); 8) Kervini, Poullan-sur-Mer, Finistère (after Halna du Fretay, 1888); 9) Coët-er-Gaff, Elven, Morbihan (after Marsille, 1913).

d'Armor; fig. $11, \mathrm{n}^{\text {os }} 5$ et 6) semblent être des imitations assez frustes avec des ailerons très larges. La pointe de Tossen-Kergourognon possède en même temps des affinités avec certaines armatures de la tombe de Graeoc 2. On retrouve les mêmes ailerons légèrement évasés et la présence d'un pédoncule naissant (fig. $11, \mathrm{n}^{\text {os }} 2$ et 5 ).

Il apparaît que les productions léonardes de type Limbabu ont diffusé sur une distance d'une soixantaine de kilomètres à vol d'oiseau. L'homogénéité et la parenté stylistique confirment cette hypothèse. Il faut admettre que ce sont les meilleurs exemplaires qui ont circulé le plus loin. Sans cela, on ne peut expliquer que l'armature de type Limbabu avec les ailerons les plus développés (23,2 mm) se trouve hors du Léon (fig. 11, $\left.\mathrm{n}^{\mathrm{o}} 4\right)$.

\section{Éléments de technologie}

Les pointes de flèches de type armoricain sont des produits finis réalisés avec une retouche extrêmement soignée. On ne connaît que les ultimes phases des chaînes opératoires. Dans un cas, la retouche courte et envahissante a permis d'observer la nature du support avec deux surfaces légèrement bombées (fig. 7, $\mathrm{n}^{\mathrm{o}} 30$ ). Un double bombement est relativement fréquent dans les profils de pointes de flèches. Il pourrait être un indice de l'emploi d'éclats Kombewa (Inizan et al., 1995), ceux-ci présentant l'avantage d'être réguliers et d'avoir une double convexité facilitant la retouche couvrante à la pression (S. Blanchet, com. pers.). Cela doit être nuancé, car il est généralement situé au niveau du dégagement du pédoncule, qui est la phase la plus 


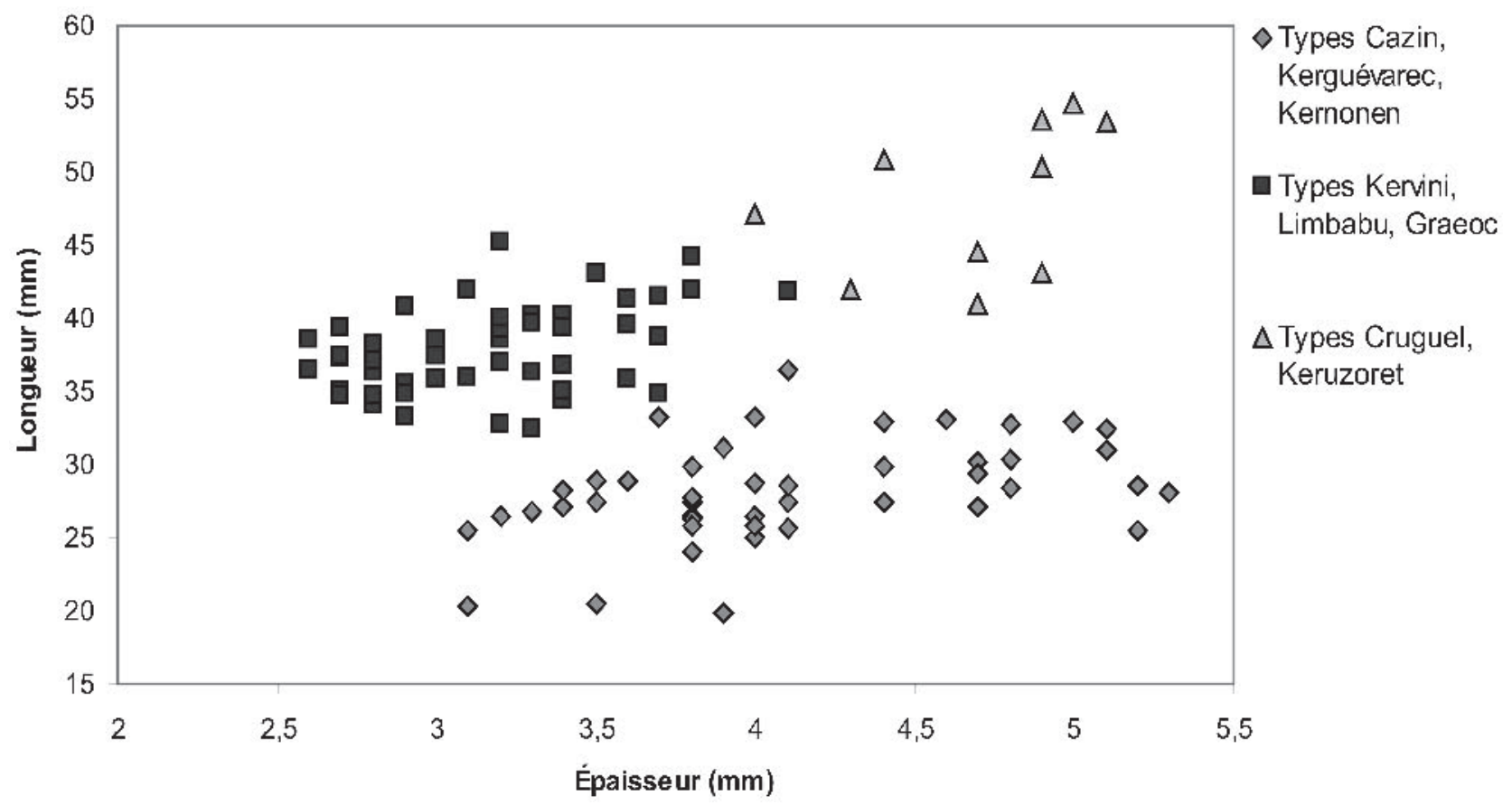

Fig. 12 - Type de pointes de flèches et épaisseur des produits finis.

Fig. 12 - Types of arrowheads and thickness of final products.

\begin{tabular}{|l|c|c|c|c|c|c|}
\hline & \multicolumn{3}{|c|}{ Étendue } & \multicolumn{3}{c|}{ Combinaison } \\
\hline \multicolumn{1}{|c|}{ Type } & Couvrante & Envahissante & Courte & Irrégulière & En chevrons & Transverse \\
\hline Cazin & $\mathbf{5}$ & 1 & - & $\mathbf{5}$ & 1 & - \\
\hline Kerguévarec & $\mathbf{3 3}(\mathbf{8 4 , 6} \%)$ & $5(12,8 \%)$ & $1(2,6 \%)$ & $\mathbf{2 1}(\mathbf{4 7 , 7} \%)$ & $19(43,2 \%)$ & $4(9,1 \%)$ \\
\hline Kernonen & $\mathbf{3 7}(\mathbf{8 4 , 1} \%)$ & $3(6,8 \%)$ & $4(9,1 \%)$ & $\mathbf{1 6}(\mathbf{4 4 , 4} \%)$ & $10(27,8 \%)$ & $10(27,8 \%)$ \\
\hline Kervini & $\mathbf{3}$ & 2 & 1 & 1 & $\mathbf{2}$ & - \\
\hline Limbabu & $\mathbf{7 9}(\mathbf{9 5 , 2} \%)$ & $4(4,8 \%)$ & - & $14(16,9 \%)$ & $31(37,3 \%)$ & $\mathbf{3 8} \mathbf{( 4 5 , 8 \% )}$ \\
\hline Graeoc & $\mathbf{4}$ & - & - & 1 & $\mathbf{2}$ & 1 \\
\hline Cruguel & $\mathbf{4}$ & - & - & - & 1 & $\mathbf{3}$ \\
\hline Keruzoret & $\mathbf{1 6}(\mathbf{1 0 0} \%)$ & - & - & $3(18,8 \%)$ & $3(18,8 \%)$ & $\mathbf{1 0}(\mathbf{6 2 , 5} \%)$ \\
\hline Total & $\mathbf{1 8 1}(\mathbf{9 1} \%)$ & $13(6,5 \%)$ & $5(2,5 \%)$ & $61(31,1 \%)$ & $69(35,2 \%)$ & $66(33,7 \%)$ \\
\hline
\end{tabular}

Tabl. 4 - Étendue et combinaison de la retouche de façonnage suivant les types de pointes de flèches.

Table 4-Extent and assemblage of shaping retouches according to types of arrowheads.

critique de la taille. La difficulté que représente ce dégagement peut avoir également contribué à laisser un bombement simple ou double (fig. 7, $\mathrm{n}^{\text {os }} 3,13,22$ et 26).

Il existe une différenciation nette entre les épaisseurs de certains types de pointes de flèches (fig. 12). Les armatures de type Kervini, Limbabu, Graeoc sont les pointes de forme ogivale les plus allongées et les plus fines. Elles se distinguent clairement des types Cazin, Kerguévarec et Kernonen qui sont plus courtes et plus épaisses. On peut supposer que ces pointes de flèches ont été réalisées sur des supports différents. La volonté de réaliser des formes ogivales plus allongées a imposé l'utilisation de supports plus fins, éclats simples ou Kombewa. Le cas des pointes de flèches triangulaires illustre l'utilisation de supports plus épais par les tailleurs, entraînant peut-être l'abandon du pédoncule. En effet les pointes triangulaires de type Keruzoret se caractérisent par l'absence de véritable pédoncule - certaines pointes présentant un petit appendice à la place (fig. $9, \mathrm{n}^{\text {os }} 3$ à 7 ).

La retouche est couvrante $(91 \%)$ et plus rarement envahissante $(6,5 \%)$ ou courte $(2,5 \%$; tabl. 4). Les séries d'ogivales courtes et moyennes (types Cazin, Kerguévarec) présentent plus fréquemment une retouche envahissante $(10,1 \%)$ ou courte $(5,6 \%$; tabl. 4$)$. Elles sont réalisées le plus souvent par un façonnage irrégulier multidirectionnel avec fréquemment plusieurs séries de retouches. Les types Limbabu et Keruzoret ont une retouche plus régulière, majoritairement transverse ou en chevrons (tabl. 4).

L'aspect technologique le plus problématique est de comprendre comment ces artisans ont pu tailler des ailerons aussi longs et fins sur des supports aussi fins, entre 2,6 et 4,1 mm d'épaisseur (fig. 12). Chaque enlèvement de façonnage du pédoncule et de son dégagement a dû constituer un moment critique dans la taille, le moindre faux pas pouvant entraîner le bris de 


\begin{tabular}{|l|c|c|}
\hline \multicolumn{1}{|c|}{ Type } & $\begin{array}{c}\text { Nombre } \\
\text { de rebroussés }\end{array}$ & $\begin{array}{c}\text { Dont dégagement } \\
\text { du pédoncule }\end{array}$ \\
\hline Cazin & 21 & $5(23,8 \%)$ \\
\hline Kerguévarec & 219 & $88(40,2 \%)$ \\
\hline Kernonen & 200 & $88(44 \%)$ \\
\hline Kervini & 35 & $8(22,8 \%)$ \\
\hline Limbabu & 390 & $84(21,5 \%)$ \\
\hline Graeoc & 17 & $6(35,3 \%)$ \\
\hline Cruguel & 30 & $10(33,3 \%)$ \\
\hline Keruzoret & 16 & $3(18,7 \%)$ \\
\hline
\end{tabular}

Tabl. 5 - Nombre et localisation des rebroussés suivant les types de pointes de flèches.

Table 5 - Number and location of hinged removals according to types of arrowheads.

la pointe de flèche. La difficulté de l'exercice est illustrée par la part importante de rebroussés occasionnés par le dégagement du pédoncule et des ailerons, qui peut totaliser jusqu'à $44 \%$ du nombre total des rebroussés (tabl. 5). Tailler des ailerons de 16,3 $\mathrm{mm}$ de longueur sur une largeur de $13,6 \mathrm{~mm}$, comme sur une pointe de Graeoc 2 (fig. 8, $n^{\circ} 3$ ), nécessite une technique et des outils particuliers. Pour cette raison, on peut écarter la percussion directe ou indirecte. Seule l'utilisation d'une pression bien maîtrisée a pu permettre de développer des ailerons longs. L'espace entre le pédoncule et les ailerons - pouvant ne pas dépasser 2 millimètres (fig. 8, $\mathrm{n}^{\mathrm{O}} 3$ ) - suppose que l'instrument utilisé possède une pointe très fine. Pour être fonctionnelle, cette pointe doit être dans un matériau suffisamment résistant. Ce genre de pointe, connue sous le nom d'alène d' "alêne», apparaît dès le Chalcolithique. En Bretagne, on en recense trois exemplaires qui proviennent de sépultures mégalithiques du Morbihan : Goërem à Gâvres, Port-Blanc à Saint-Pierre-Quiberon et Beg-er-Lann à Plœmeur (Giot et al., 1962, p. 89). L'alêne de Goërem a pu être analysée : elle est composée d'un cuivre arsénié (L'Helgouac'h, 1970). Elle a été retrouvée en même temps que deux pointes de flèches à ailerons et pédoncule équarris de type campaniforme (L'Helgouac'h, 1970, p. 252). Pour l'âge du Bronze ancien la sépulture de Kerest en Locquirec (Finistère) a fourni une alêne de 37 millimètres et de section losangique (Le Roux, 1983, p. 317). À Kingsmead Quarry (Horton, Berkshire, Angleterre), un dépôt exceptionnel renfermait la panoplie probable d'un tailleur de pointes de flèches : 8 pointes de flèches, 5 éclats façonnés ou non, 1 alêne en cuivre et 1 aiguisoir en grès (Wessex Archaeology, 2009). L'association d'alênes avec des pointes de flèches montre qu'elles ont pu servir à la taille du silex, sans qu'on puisse toutefois exclure d'autres fonctions. Une trace cuivreuse située dans le creux d'un enlèvement d'une armature de Graeoc 2 (fig. 8, $n^{\circ} 3$ ) pourrait s'expliquer par une retouche à la pression avec une alêne en bronze ou en cuivre (Nicolas, 2008).

Les éléments de technologie ne sont pas assez consistants pour permettre de reconstituer l'ensemble des maillons de la chaîne opératoire. Du point de vue des techniques, la retouche des ailerons pourrait avoir nécessité l'utilisation d'une alêne fine et allongée, en cuivre ou probablement en bronze. D'autres instruments devaient sûrement être utilisés au cours des différentes étapes, du débitage du support au produit fini, mais il est impossible de les mettre en évidence.

\section{Styles, artisans et productions spécialisées}

Plusieurs séries présentent un ou plusieurs styles de taille, qui peuvent être interprétés comme l'activité de plusieurs artisans ou ateliers. L'homogénéité stylistique des types de pointes de flèches peut être estimée à partir du nombre de sous-types (tabl. 6). Les types Kernonen, Limbabu, Graeoc et Keruzoret possèdent une fréquence moins grande de sous-types. Cette variabilité plus faible s'accompagne d'un phénomène de standardisation qui s'exprime à travers le sous-type 332, correspondant à un pédoncule rentrant et à des ailerons droits taillés en oblique forte. Le sous-type 332 se trouve majoritairement sur les types Kernonen (59,6 \%) et Limbabu $(58,1 \%)$. Pour le type Keruzoret, qui ne possède pas de pédoncule, c'est le sous-type 112, avec des ailerons droits taillés en oblique faible, qui marque la standardisation (tabl. 6). La distinction de ces types dans la standardisation trouve des parallèles dans les méthodes de façonnage (tabl. 4) et les matières premières (tabl. 3).

La morphologie, les aspects technologiques et les matières premières permettent de mettre en évidence des productions hétérogènes (types Cazin, Kerguévarec, Kernonen) et homogènes (types Kervini, Limbabu, Graeoc, Cruguel, Keruzoret). On peut se demander si ces types ne répondent pas à deux logiques dans la constitution des dépôts funéraires. Les productions hétérogènes pourraient correspondre à la multiplicité des origines et, donc, des tailleurs, et signaler des phénomènes d'échanges et d'accumulation dans la durée. Les types homogènes supposent en revanche une unité de taille, des tailleurs et possiblement du moment de la taille. L'homogénéité des dépôts et la production léonarde de type Limbabu permettent de supposer l'existence d'un atelier composé au minimum de deux ou trois personnes. Cet atelier devait être constitué d'au moins un tailleur expérimenté et d'autres tailleurs, auxquels il devait transmettre son savoir-faire. Tailler des armatures de type Limbabu a dû nécessiter un long apprentissage, et les meilleurs expérimentateurs actuels se demandent encore comment elles ont pu être taillées (J. Pelegrin, com. pers.).

En l'absence de collections importantes d'industries lithiques de l'âge du Bronze ancien, il est difficile de mettre en évidence des savoir-faire locaux. À l'extrémité orientale du Massif armoricain, la fouille du site d'habitat de l'île de Tatihou (Manche) montre une industrie lithique de tradition néolithique (Marcigny et Ghesquière, 2003). Quant aux pointes de flèches trouvées lors de prospections, elles sont assez frustes, et leur attribution chronologique demeure imprécise. Les prospections menées par F. Le Provost sur le plateau du Collédic (Saint-Nicolas-du-Pélem, Côtes-d'Armor) 


\begin{tabular}{|c|c|c|c|c|c|c|c|c|c|}
\hline \multicolumn{2}{|c|}{ Sous-type } & \multirow[t]{2}{*}{ 墕 } & \multirow[t]{2}{*}{ 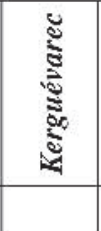 } & \multirow[t]{2}{*}{ 苞 } & \multirow[t]{2}{*}{ 玆 } & \multirow[t]{2}{*}{ 氙 } & \multirow[t]{2}{*}{$\stackrel{\Xi}{\Xi}$} & \multirow[t]{2}{*}{ 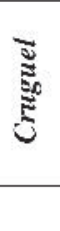 } & \multirow{2}{*}{ 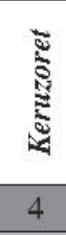 } \\
\hline$\ldots$ & 112 & & & & & & & & \\
\hline 1 & 122 & & & & & & & & 1 \\
\hline L & 152 & & & & & & & & 3 \\
\hline $1 \ldots$ & 212 & & & & & 1 & & & \\
\hline … & 222 & & & & & & & 1 & \\
\hline$\sum^{\prime} 2 \vdots$ & 231 & & & & & 3 & & 1 & \\
\hline $2-21$ & 232 & & & & & 2 & & & \\
\hline $2 \cdots$ & 252 & & & & & 1 & & & \\
\hline$\vdots .7 ! \vdots$ & 311 & & & & & 2 & & & \\
\hline$\ldots V$ & 312 & & & 1 & & 4 & & & \\
\hline$\vdots 0 \vee 1$ & 331 & 1 & 1 & 1 & & 4 & & & \\
\hline$\checkmark \mathrm{A}$ & 332 & & 9 & 31 & 4 & 32 & 2 & 1 & \\
\hline$\sim 21$ & 333 & & 2 & & & 1 & & & \\
\hline$i-V^{\prime} i$ & 341 & & 2 & 1 & & & & & \\
\hline,$\sqrt{1}$ & 342 & & 1 & 4 & & 1 & & & \\
\hline LViv & 343 & & 1 & 1 & & & & & \\
\hline$n \Omega$ & 351 & & 1 & & & 2 & & & \\
\hline 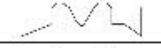 & 352 & & 1 & 2 & & 1 & & & \\
\hline $1-\sqrt{1} \mid$ & 353 & & 1 & 1 & & & & & \\
\hline 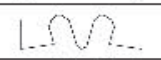 & 413 & & & 1 & & & & & \\
\hline 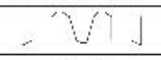 & 422 & & 1 & & & & & & \\
\hline 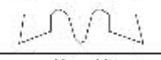 & 431 & & 2 & & & & & & \\
\hline$\checkmark 1$ & 432 & & 2 & 2 & & 1 & & & \\
\hline$i-r-i$ & 441 & & 2 & & & & & & \\
\hline 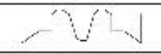 & 442 & & 1 & & & & & & \\
\hline 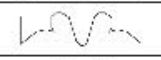 & 443 & & 2 & & & & & & \\
\hline $1 \int$ & 452 & & 1 & & & & & & \\
\hline 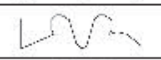 & 453 & & 3 & 1 & & & & & \\
\hline 1.1$)$ & 513 & & & 1 & & & & & \\
\hline $\int \sqrt{ }$ & 522 & & & 1 & & & & & \\
\hline$\vdots^{\prime}$ & 531 & & 1 & & & & & & \\
\hline 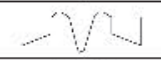 & 532 & 1 & 5 & 1 & & & & & \\
\hline $\int ध$ & 533 & & & 1 & & & & & \\
\hline$i r$ & 541 & 1 & 1 & & & & & & \\
\hline$-\Omega 1$ & 542 & & 1 & 2 & & & & & \\
\hline $\int \mathrm{l}$ & 543 & 1 & 2 & & & & & & \\
\hline 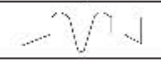 & 552 & 1 & 1 & & & & & & \\
\hline & Indéterminés & & & 6 & & 16 & & & \\
\hline & Total & 5 & 44 & 58 & 4 & 71 & 2 & 3 & 8 \\
\hline & $\begin{array}{c}\text { Fréquence des } \\
\text { sous-types }\end{array}$ & I & 0,52 & 0,31 & 0,25 & 0,24 & 0,5 & I & 0,38 \\
\hline & $\begin{array}{c}\text { Part du sous-type } \\
332\end{array}$ & & $20,4 \%$ & $59,6 \%$ & & $58,1 \%$ & & & \\
\hline & $\begin{array}{l}\text { Part du sous-type } \\
112\end{array}$ & & & & & & & & $50 \%$ \\
\hline
\end{tabular}

Nombre de pointes de flèches

\section{1 \\ 2 à 5 \\ 6 à 10 \\ $>10$}

Tabl. 6 - Tableau de correspondance entre les types et la morphologie des ailerons et du pédoncule. Fréquence des sous-types $=$ nombre de soustypes/pointes de flèches.

Table 6-Table of correspondences of types and barbs and tang morphology. Subtypes frequency $=$ number of subtypes/number of arrowheads. 
montrent une grande variété de types qui offrent un aperçu des pointes de flèches fonctionnelles du Chalcolithique-âge du Bronze ancien (Le Provost et al., 1972). On peut se demander si une partie de ces armatures ne sont pas contemporaines des pointes de flèches de type armoricain (Briard, 1976, p. 38). Dans ce cas, une production domestique d'armatures de flèches destinées au tir à l'arc aurait coexisté avec une production de pointes hors du commun vouées à d'autres usages, parmi lesquels celui de constituer certains dépôts funéraires.

Les fouilles récentes montrent que les pointes de flèches sont majoritairement intactes, mais que certaines devaient être brisées au moment du dépôt, comme dans la tombe de Prat-ar-Simon-Pella (Lannilis, Finistère; Le Goffic et Nallier, 2008). Les 10 pointes de flèches de Keruzoret, conservées dans trois musées différents (musée d'Archéologie nationale, musée de Préhistoire finistérienne à Penmarc'h, musée des Jacobins à Morlaix), possèdent, sur leurs surfaces, un poli qui atteste d'une usure du silex après la retouche bifaciale couvrante. Cette usure se répète de manière similaire sur les deux faces des 10 pointes, excluant tout phénomène taphonomique. Elle pourrait éventuellement correspondre à un poli de fourreau (fig. 9). Cela serait la marque d'une utilisation de ces pointes de flèches avant leur dépôt dans les sépultures. Dans certains cas, la fouille a montré que les pointes de flèches étaient soigneusement déposées dans des contenants en matériaux périssables, comme un coffret en bois de chêne à Kernonen (Plouvorn, Finistère; Huard, 1970). Cela semble confirmer que ces armatures assuraient une fonction particulière, qui reste à déterminer.

La production des pointes de type armoricain répond à des logiques d'accumulation (types Cazin, Kerguévarec, Kernonen) ou de commande pour les productions homogènes (types Kervini, Limbabu, Graeoc, Cruguel, Keruzoret). La production léonarde de type Limbabu atteste l'existence d'un atelier composé de tailleurs possédant un savoir-faire spécialisé. La qualité d'exécution de ces objets et l'utilisation systématique du silex blond translucide montrent que cette production possédait un statut valorisé. Posséder un lot d'armatures de pointes de flèches de type armoricain devait sans aucun doute souligner un statut social élevé. Il n'est pas anodin que ces pointes soient associées à des poignards, fréquemment à des haches en bronze et plus rarement à des objets en matières précieuses, or, argent, ambre ou jais. Les clous d'or inframillimétriques décorant le manche des poignards ont été découverts uniquement dans des sépultures à pointes de flèches.

\section{DU CAMPANIFORME À L'ÂGE DU BRONZE ANCIEN : LA POINTE DE FLÈCHE ET LES BIENS SOCIALEMENT VALORISÉS}

\section{Typochronologie des tumulus à pointes de flèches}

L'étude a permis de mettre en évidence huit types de pointes de flèches. On peut se demander si ces différences typologiques sont dues à une répartition spatiale ou à une évolution chronologique. Pour cela, il est nécessaire d'observer les différents types d'objets associés aux pointes de flèches.

La typologie des mobiliers associés prend en compte les éléments que l'on retrouve deux fois au minimum et qui sont susceptibles d'être sériés : les poignards en bronze et leur décoration de clous d'or, les haches en bronze, les brassards d'archer. La typologie des poignards en bronze utilisée ici (fig. 13) reprend les travaux de G. Gallay (1981) et de S. Needham (2000). Malgré la corrosion de certains exemplaires, nous avons affiné cette typologie pour le type Rumédon en tenant compte du nombre de filets. Nous distinguerons un type Rumédon 1 à deux filets et un type Rumédon 2 à trois filets (fig. 8, $\mathrm{n}^{\circ} 17$ ). Le type Trévérec a un renflement central qui est facilement identifiable. Dans le nord du Finistère, les poignards de type Bourbriac possèdent deux larges filets (fig. 8, $\mathrm{n}^{\circ} 18$ et fig. 9, $\left.\mathrm{n}^{\mathrm{o}} 11\right)$. Les haches en métal présentent toutes de légers rebords (Briard, 1965, p. 60). Deux tumulus ont livré deux brassards d'archer, l'un en jais à Kerguévarec (Plouyé, Finistère; Briard et al., 1994) et l'autre en ambre à Kernonen (Plouvorn, Finistère; Briard, 1970a).

Ces associations montrent une évolution dans la composition des dépôts funéraires, sans qu'il y ait de grandes ruptures. Il y a trois groupes d'association de pointes de flèches: types Cazin-KerguévarecKernonen, types Kervini-Limbabu-Graeoc, types Cruguel-Keruzoret (fig. 13). Dans cette sériation, les pointes de flèches apparaissent véritablement comme un marqueur chronologique. La typochronologie des sépultures à pointes de flèches s'appuie sur les quatre séries définies par S. Needham (2000, p. 158-159). La redéfinition des types de pointes de type armoricain permet de la préciser. Chaque série correspond à un groupe de pointes de flèches. Les séries 1 et 2 se distinguent par la présence ou l'absence d'un brassard d'archer. Le cas de la sépulture de Cazin (Plouigneau, Finistère) reste difficile d'interprétation en raison de l'état de corrosion très avancé du mobilier métallique. Seuls les restes d'un probable poignard ont pu être observés à la fouille (fig. 7). Il est possible que d'autres objets métalliques aient fait partie du dépôt funéraire (Giot, 1977). Les haches caractérisent les séries 2 et 3 . Le poignard type Bourbriac est spécifique de la série 4. Une subdivision a été apportée à l'intérieur de la série 4 en fonction des types de pointes de flèches (fig. 13).

La typochronologie montre une réduction progressive de la variété des objets et de leur quantité (fig. 13). La série 2 est composée des tombes de Kernonen (Plouvorn, Finistère) et de Kerguévarec (Plouyé, Finistère), qui possèdent tous les types d'objets dans leur quantité maximale. Ce phénomène se retrouve dans la constitution des dépôts de pointes de flèches, qui ont une grande variété typologique. Dès la série 3, le brassard d'archer disparait. Les séries 3 et 4A voient une fréquence moins grande des haches, qui disparaissent totalement dans la série 4B. Enfin, les assemblages montrent une réduction progressive du nombre de 
poignards et de pointes de flèches. Les dépôts paraissent réduits à leur plus stricte expression, le poignard et la pointe de flèche. On peut penser que, dès la série 1 , ce sont le poignard et la pointe de flèche qui soulignent le caractère spécifique de ces sépultures, comme cela a depuis longtemps été évoqué (Micault, 1881; Aveneau de la Grancière, 1899; Martin, 1900; Briard, 1984).
La différenciation stricte entre les associations de types d'armatures de flèches dans notre corpus peut être la marque de la production léonarde de type Limbabu. La mise en place d'un atelier spécialisé pourrait expliquer la diffusion rapide et l'importance de cette production dans les dépôts funéraires du nord du Finistère. Cela expliquerait la part importante que prennent les séries 3 et $4 \mathrm{~A}$ dans cette partie de la Bretagne.

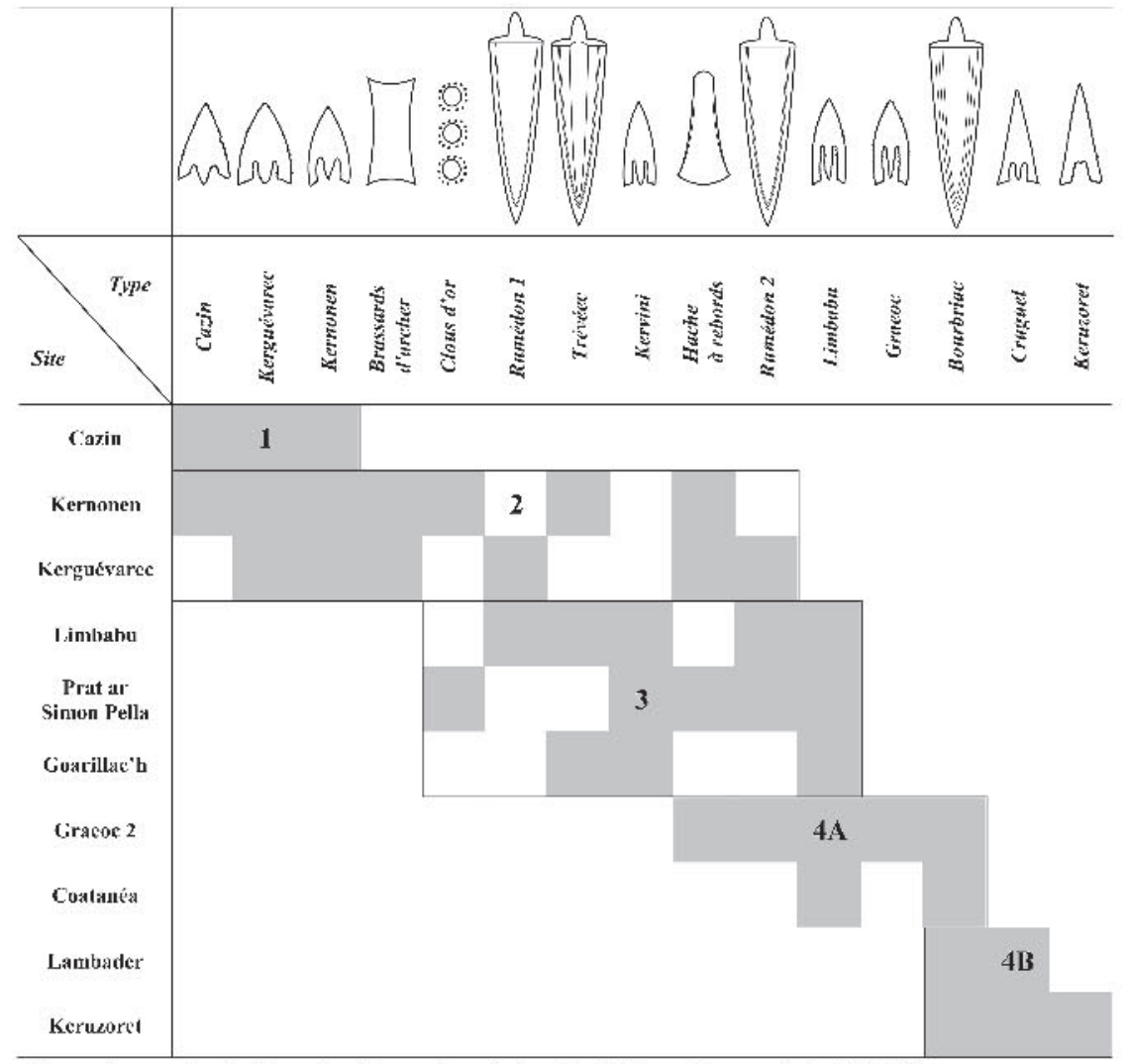

Typochronologie des sépultures à pointes de flèches du nord du Finistère.

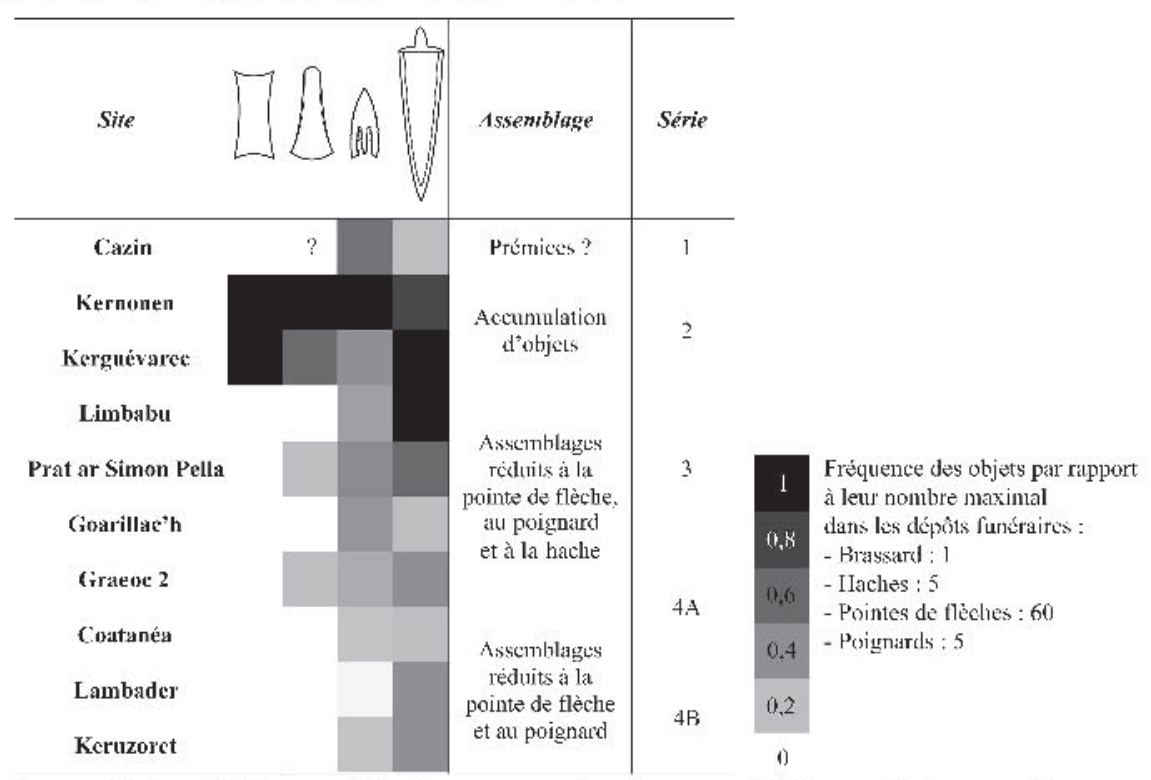

Accumulation d'objets et réduction progressive des types d'objets et de leur nombre ( séries d'après Needham, 2000).

Fig. 13 - Chronologie des sépultures à pointes de flèches.

Fig. 13 - Chronology of graves with arrowheads. 
La série 2 correspond à un horizon où se trouvent concentrés la plupart des types d'objets de l'âge du Bronze ancien de Basse-Bretagne. Cette accumulation d'objets se traduit par l'emploi de matières précieuses ou exotiques, telles que l'or, l'argent, l'ambre et le jais (Needham, 2000, p. 160). La série 2 se distingue également par la quantité d'objets accumulés. Les séries 3 à 4B marquent un abandon progressif de certains types d'objets et une réduction de leur nombre pour se focaliser sur le poignard et la pointe de flèche.

\section{Vie et mort de la pointe de flèche de type armoricain}

Les problèmes de chronologie absolue sont importants pour l'âge du Bronze ancien de Basse-Bretagne, en raison notamment d'un nombre trop faible de datations ${ }^{14} \mathrm{C}$ fiables. Après un inventaire des dates radiocarbone, J.-L. Voruz propose même de faire remonter les débuts de l'âge du Bronze ancien breton à la moitié du III ${ }^{\mathrm{e}}$ millénaire (Voruz, 1996, p. 126). L'auteur se garde toutefois de vérifier la fiabilité de ces dates (Voruz, 1996, p. 129). L'ensemble des datations montrerait que l'âge du Bronze ancien émerge pendant le XXIII ${ }^{\mathrm{e}}$ siècle avant notre ère (Voruz, 1996, p. 122). Ce calage chronologique n'est pas sans poser des problèmes dans les rapports qu'entretiennent les groupes de l'âge du Bronze ancien et ceux du Campaniforme (Salanova, 2000, p. 163). Dans le même temps, les datations ${ }^{14} \mathrm{C}$ du Campaniforme s'étendent jusqu'à la fin du $\mathrm{III}^{\mathrm{e}}$ millénaire. Campaniforme et âge du Bronze ancien restent profondément entremêlés au niveau des datations absolues. Ce problème est, entre autres, dû à la courbe de calibration des dates radiocarbone, «le millénaire des dates comprises entre 4000 et 3000 BP ayant duré en réalité plus de 1200 ans » (Voruz, 1996, p. 107).

En Bretagne une relation culturelle entre Campaniforme et âge du Bronze ancien est discutée depuis une cinquantaine d'années (Briard et L'Helgouac'h, 1957; Briard 1976; L'Helgouac'h, 2001 ; Salanova, 2007). Il semble bien qu'il y ait une filiation entre la panoplie campaniforme et l'assemblage armoricain dans les dépôts funéraires. Au vu de la typochronologie des pointes de flèches de type armoricain, on peut se demander si les types anciens (Cazin, Kerguévarec et Kernonen : fig. 13 et 14) peuvent fournir une clé pour préciser ces rapports entre Campaniforme et âge du Bronze ancien.

Il n'a pas été réalisé d'inventaire exhaustif des pointes de flèches chalcolithiques et campaniformes. Dès 1900, A. Martin souligne la parenté entre les armatures découvertes dans les dolmens et les pointes de flèches de type armoricain. Il relève que les meilleures comparaisons sont obtenues avec des pointes de flèches issues de sépultures mégalithiques qui ont également livré du mobilier métallique (Martin, 1900, p. 165166). Ces pointes de flèches, le mobilier métallique en or ou en cuivre et les gobelets céramiques sont à relier à la réutilisation des sépultures mégalithiques par les groupes campaniformes (L'Helgouac'h, 2001, p. 297).
L. Salanova précise que, dans les régions où les ossements sont conservés, le mobilier campaniforme se rattache nettement à des sujets individualisés au sein du caveau mégalithique (Salanova 2007, p. 214). Toutes les pointes de flèches sélectionnées sont à pédoncule et/ou ailerons équarris, armatures reconnues comme typique du Campaniforme (Guyodo, 2001, p. 306; Bailly 2002, p. 265; Renard 2003, p. 23; Furestier, 2005 , p. 261). Elles sont issues de sépultures mégalithiques et trouvées en même temps que du mobilier métallique et des gobelets campaniformes (fig. 14, $\mathrm{n}^{\text {os }} 1$ à 5). De plus, quatre sépultures individuelles attribuables au Campaniforme ont livré des pointes de flèches (fig. 14, n ${ }^{\text {os }} 6$ à 10).

La plupart de ces pointes de flèches campaniformes présentent un équarrissement du pédoncule et/ou des ailerons (fig. 14, $\mathrm{n}^{\text {os }}$ 1, 2, 4 à 6, 10 et 11). Elles adoptent majoritairement une forme triangulaire (fig. 14, $\mathrm{n}^{\text {os }} 1$ à 3 et 6 et 9), mais aussi subtriangulaire (fig. 14, $n^{\text {os }} 5$ et 10) ou ogivale (fig. 14, $\mathrm{n}^{\circ} 4$ ). Deux possèdent des ailerons taillés en oblique (fig. 14, $n^{\text {os }} 2$ et 4 ), et deux autres possèdent un pédoncule dépassant en $\langle\mathrm{V}$ 》 (fig. $14, \mathrm{n}^{\text {os }} 9$ et 10 ). Il existe des armatures triangulaires plus frustes qui sont pédonculées (fig. 14, $\mathrm{n}^{\text {os }} 3$ et 8) ou qui possèdent un pédoncule et des ailerons (fig. 14, $\mathrm{n}^{\mathrm{O}} 7$ ). Les formes subtriangulaires et ogivales sont associées à des ailerons taillés en oblique dans le cas de la pointe du cairn de Barnenez (Plouzéoc'h, Finistère : fig. 14, $\mathrm{n}^{\circ} 4$ ) et à un pédoncule dépassant en «V » pour 1'armature du tumulus de Kermenhir (Poullan-sur-Mer, Finistère; fig. 14, $\mathrm{n}^{\circ} 10$ ). Tous les éléments typologiques de la pointe de flèche de type armoricain (formes subtriangulaire et ogivale, pédoncule en $« \mathrm{~V} »$, ailerons taillés en oblique) sont présents dans le Campaniforme breton. Toutefois, certains éléments comme le pédoncule en « $\mathrm{V} »$ existent depuis le Néolithique final (Guyodo, 2001; Renard, 2003).

Le cas des pointes de flèches (fig. $14, \mathrm{n}^{\text {os }} 14$ et 15 ) provenant du tumulus de Lothéa (Quimperlé, Finistère; Taylor et al., 1846) montre la coexistence d'armatures à ailerons équarris et de poignards de type armoricain (Briard et Mohen, 1974). La sépulture a également fourni une pendeloque, probablement en jadéitite, qui renvoie également à une tradition néolithique (Briard et Mohen, 1974). Deux pointes de flèches de Lothéa possèdent la même morphologie que les pointes de flèches de la sépulture campaniforme de Kermenhir (Poullan-sur-Mer, Finistère; $\operatorname{archives~du~Chatellier~}^{1}$ ) : une pointe triangulaire (fig. $14, n^{\text {os }} 9$ et 14 ) et une armature subtriangulaire ayant des bords légèrement convexes (fig. 14, $\mathrm{n}^{\text {os }} 10$ et 15 ).

Le type Cazin montre le plus d'affinités avec les armatures campaniformes. La plupart des armatures de ce type possèdent un pédoncule taillé en « $\mathrm{V} »$ nettement dépassant et des ailerons courts (fig. 7, $\mathrm{n}^{\text {os }} 2$ et 26, fig. 14, $\mathrm{n}^{\text {os }} 12$ à 14). Le type Cazin entretient également une certaine parenté avec les deux pointes de flèches de Kermenhir (Poullan-sur-Mer, Finistère; fig. 14, $\mathrm{n}^{\text {os }} 9$ et 10). Le dépassement prononcé du pédoncule et la forme subtriangulaire apparaissent comme un archaïsme issu des pointes de flèches campaniformes. Les 13 pointes de type Cazin, qui ont pu être 
<smiles>CCCCCCCCCCCCCCCC</smiles>

Élaboration d'un standard

stylistique : le sous-type 332

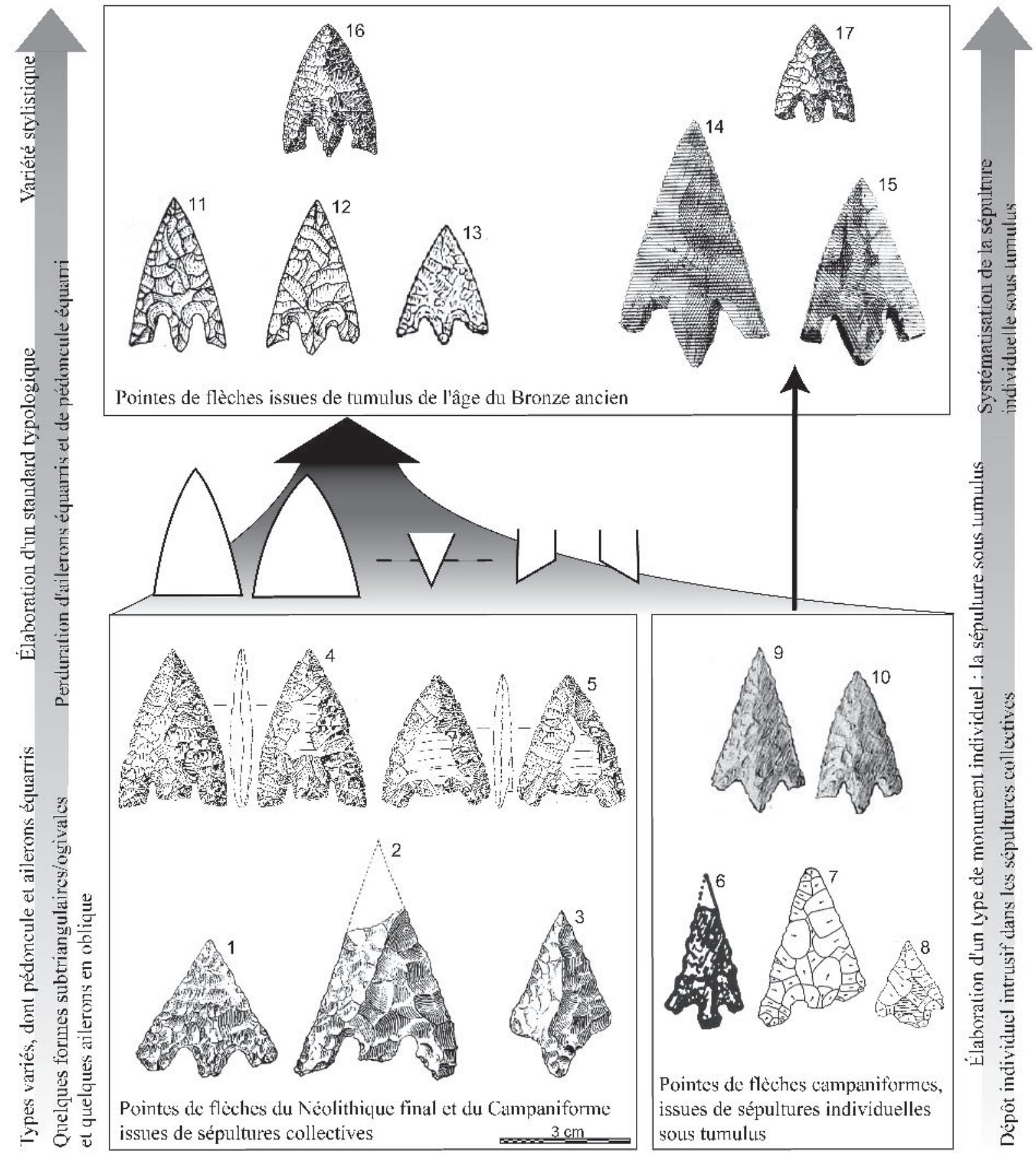

Fig. 14 - Genèse de la pointe de flèche de type armoricain : $\mathrm{n}^{\circ}$ 1) allée couverte de Kerandrèze, Moëlan-sur-Mer, Finistère (d'après Martin, 1900); $\mathrm{n}^{\text {os }} 2$ et 3 ) allée couverte du Run, Tréffiagat, Finistère (d'après Martin, 1900); $\mathrm{n}^{\circ} 4$ ) chambre D, cairn de Barnenez, Plouzéoc'h, Finistère (dessin C. Nicolas); $\mathrm{n}^{\circ}$ 5) allée couverte du Kernic, Plouescat (dessin C. Nicolas); $\mathrm{n}^{\circ}$ 6) tumulus de Crubelz, Belz, Morbihan (d'après Closmadeuc, 1864); $\mathrm{n}^{\text {os }} 7$ et 8 ) tumulus de Coatjou-Glas, Plonéis, Finistère (d'après Chaigneau-Normand, 1994); ${ }^{\text {os }} 9$ et 10) tumulus de Kermenhir, Poullan-sur-Mer, Finistère (d'après Chatellier, archives); $\mathrm{n}^{\mathrm{os}} 11$ et 12) tumulus de Kernonen, Plouvorn, Finistère (d'après Briard, 1970a); $\mathrm{n}^{\circ} 13$ ) hors de la sépulture, tumulus de Brun Bras, Saint-Adrien, Côtes-d'Armor (d'après Briard, 1976); $\mathrm{n}^{\mathrm{os}} 14$ et 15) tumulus de Lothéa, Quimperlé, Finistère (d'après Taylor et al., 1846); $\mathrm{n}^{\text {os }} 16$ et 17) tumulus de Cazin, Plouigneau, Finistère (dessin C. Nicolas).

Fig. 14 - Genesis of Armorican-type arrowheads: 1) Kerandrèze gallery grave, Moëlan-sur-Mer, Finistère (after Martin, 1900); 2 and 3) Run gallery grave, Tréffiagat, Finistère (after Martin, 1900); 4) chamber D, Barnenez cairn, Plouzéoc'h, Finistère (drawing C. Nicolas); 5) Kernic gallery grave, Plouescat (drawing C. Nicolas); 6) Crubelz barrow, Belz, Morbihan (after Closmadeuc, 1864); 7 and 8) Coatjou-Glas barrow, Plonéis, Finistère (after Chaigneau-Normand, 1994); 9 and 10) Kermenhir barrow, Poullan-sur-Mer, Finistère (after Chatellier, archives); 11 and 12) Kernonen barrow, Plouvorn, Finistère (after Briard, 1970a); 13) out of grave, Brun Bras barrow, Saint-Adrien, Côtes-d'Armor (after Briard, 1976); 14 and 15) Lothéa barrow, Quimperlé, Finistère (after Taylor et al., 1846); 16 and 17) Cazin barrow, Plouigneau, Finistère (drawings C. Nicolas). 
référencées pour l'ensemble de la Basse-Bretagne constituent un ensemble relativement faible (Nicolas, 2008). Le type Cazin apparaît comme une sorte de prototype de la pointe de flèche de type armoricain : il en possède tous les éléments typologiques tout en conservant des aspects des armatures campaniformes. À l'échelle du nord du Finistère, le pédoncule dépassant est plus fréquemment associé au type Cazin (tabl. 6). Il paraît assez marginal dans les types Kerguévarec et Kernonen (tabl. 6). On peut considérer que, parfois, des accidents de taille conduisent le tailleur à réaliser des ailerons plus courts et, donc, un pédoncule dépassant, mais ces accidents sont statistiquement négligeables.

La pointe de flèche de Barnenez (Plouzéoc'h, Finistère; Giot, 1987, fig. nº 6) montre la préexistence de la forme ogivale et des ailerons taillés en oblique dans les armatures campaniformes. La forme ogivale va composer la majorité des pointes de flèches de type armoricain avec les types Kerguévarec (fig. 14, $\mathrm{n}^{\circ} 16$ ), Kernonen, Kervini et Limbabu. Une armature de la sépulture de Cazin (Plouigneau, Finistère) présente un pédoncule équarri en forme de trapèze (fig. 14, $\mathrm{n}^{\circ} 17$ ). L'interprétation de cette armature est problématique. Il peut s'agir simplement d'un pédoncule équarri ou d'un pédoncule en «V $\mathrm{V}$ qui a cassé à la taille et a été retouché par la suite. Dans ces deux cas, il semble bien que des traits campaniformes puissent persister soit par évolution progressive, soit par accident.

Tous les éléments typologiques concordent pour montrer une filiation locale entre les pointes de flèches campaniformes et celles de type armoricain. On peut mentionner que les deux armatures campaniformes qui ont pu être étudiées (fig. 14, $\mathrm{n}^{\text {os }} 4$ et 5) ont reçu un façonnage court ou envahissant sur au moins une des faces. Le façonnage court ou envahissant caractérise également certaines pointes de flèches des séries 1 et 2 (types Cazin, Kerguévarec et Kernonen : tabl. 4). La forme subtriangulaire du prototype Cazin va passer à une convexité plus affirmée avec les formes ogivales des types Kerguévarec et Kernonen. La typochronologie des pointes de flèches montre également une évolution constante de la morphologie des pointes de flèches de type armoricain (fig. 13). Un phénomène de standardisation du pédoncule et des ailerons est visible à travers le sous-type 332 (tabl. 6 et fig. 14). Il est confirmé par la technologie avec l'emploi probable de supports différents et une évolution des méthodes de façonnage. Pour la série 3, la matière première montre une rupture nette par l'emploi systématique du silex blond translucide (tabl. 3 et fig. 13). Enfin, on peut associer l'évolution de la pointe de flèche campaniforme à la mise en place de la sépulture individuelle sous tumulus. Le développement de la production de pointes de flèches semble connaître une dynamique parallèle à l'individualisation progressive des personnes dans les pratiques funéraires (fig. 14).

Des séries 1 à $4 \mathrm{~A}$, on recherche un allongement de la pointe et des ailerons, qui s'exprime à travers le type Limbabu. On peut souligner que, dès la série 2, des pointes de flèches sont très proches du type Limbabu. Certaines armatures du coffret $n^{\circ} 3$ du tumulus de
Kernonen (Plouvorn, Finistère) arrivent aux confins des limites typologiques (Briard, 1970a). L'allongement de la forme ogivale et des ailerons trouve sa pleine expression avec le type Limbabu, qui constitue la moitié des dépôts funéraires. Le discret type Graeoc apparaît comme une variante du type Limbabu avec une forme ogivale outrepassée (fig. 8, $n^{\text {os }} 1$ et 2). Dans la série 4A, le type Graeoc illustre l'ultime évolution morphologique de la forme ogivale par une exagération de la largeur médiane. On pourrait parler de «phénomène baroque ». Le type Graeoc apparaît seulement dans la série de la sépulture de Graeoc 2 (SaintVougay, Finistère), qui est caractérisée également par des armatures de type Limbabu particulières. Le cas de la pointe de flèche $\mathrm{n}^{\circ} 3$ de la tombe de Graeoc 2, avec un rapport L/l unique et inégalé de 3,32, témoigne d'un exploit technique et d'un art qui est en recherche d'innovations stylistiques et d'originalité (fig. 8, $\mathrm{n}^{\mathrm{O}} 3$ ). Un renouveau intervient avec la série 4B et l'apparition de pointes de flèches triangulaires (types Cruguel, Keruoret : fig. 9). La morphologie des pointes revient à une forme géométrique plus simplifiée avec des dimensions plus grandes. Les ailerons peuvent encore être longs, mais ils ont tendance à se raccourcir. Enfin le type Keruzoret illustre l'abandon du pédoncule et clôt le phénomène des pointes de flèches de type armoricain avec la série éponyme de Keruzoret (Plouvorn, Finistère).

Dans les dépôts funéraires, la disparition de la pointe de flèche en silex marque la fin d'une tradition néolithique. Elle met en lumière des changements de mode et la mise en place d'une nouvelle culture. L'âge du Bronze moyen verra la multiplication des objets métalliques et de leurs dépôts non funéraires (Briard, 1965).

\section{La pointe de flèche et les biens socialement valorisés}

On peut se demander si la parenté entre Campaniforme et âge du Bronze ancien s'observe uniquement pour les pointes de flèches ou si elle est le résultat d'une dynamique plus générale. Les rapports entre Campaniforme et Bronze ancien en Basse-Bretagne ont longtemps été jugés peu signifiants. Les sépultures à pointes de flèches ont été considérées comme l'élément le plus exogène de l'âge du Bronze ancien breton (Briard, 1984, p. 191). Généralisation de la sépulture individuelle, architectures funéraires et culture matérielle ont été expliquées par un apport extérieur (Briard, 1984, p. 193). Cette hypothèse a pu être nuancée par A. Balquet (2001, p. 50), mais elle a été illustrée récemment par un article posthume de J. Briard (2005). Du point de vue de la céramique, on reconnaît quelques vases épicampaniformes (Briard, 1984, p. 115-118; fig. 15, $\mathrm{n}^{\circ} 47$ ), mais le corpus n'est pas suffisant pour justifier une véritable parenté avec le Campaniforme (L'Helgouac'h, 2001, p. 296) et il marque une résurgence tardive du vieux fonds néolithique (Briard, 1984, p. 111). Dans cette logique de rupture, l'architecture funéraire prouverait la mise en place d'une nouvelle 
culture (Briard, 1984, p. 191). On peut admettre que l'âge du Bronze ancien voie l'apparition des cercueils en bois, comme à Cazin (Plouigneau, Finistère; Giot, 1977; fig. 7). Un des arguments de la rupture est la réutilisation de monuments mégalithiques et de mobiliers du Néolithique (Briard, 1984, p. 191). La pierre angulaire de ce raisonnement est la déesse-mère de Kersandy (Plouhinec, Finistère), une statue-menhir néolithique réemployée comme dalle de couverture dans un tumulus à pointes de flèches (Briard, 1977b; Briard et al., 1982, p. 37). Les arguments de J. Briard pour évoquer une rupture avec le Néolithique se résument à un net changement culturel et rituel, cela tout en admettant quelques liens avec le Campaniforme.

Ce schéma historique est le fruit d'une archéologie des peuples (Briard, 1984, p. 204), qui semble mal adaptée pour des périodes si anciennes. Certains éléments qui mettent en évidence la rupture avec le Néolithique peuvent être inversés et déconstruits. La réutilisation de monuments mégalithiques apparaît comme un trait de cette culture (L'Helgouac'h, 2001; Salanova, 2007) et se poursuit à l'âge du Bronze ancien, comme le montre la perle en faïence retrouvée dans le dolmen de Parc-Guren (Crac'h, Morbihan; Briard, 1984, p. 143). La plupart des intrusions campaniformes dans les sépultures collectives correspondent à des inhumations de sujets individualisés (Salanova, 2007, p. 214). D'ailleurs, la sépulture individuelle sous tumulus apparaît dès le Campaniforme (fig. 2).

La chronologie des sépultures et l'utilisation des archives P. du Chatellier permettent de préciser les relations qu'entretiennent les cultures matérielles du Campaniforme et celles de l'âge du Bronze ancien. Nous avons réalisé un inventaire de toutes les catégories d'objets qui ont été trouvées dans les dépôts funéraires campaniformes et de l'âge du Bronze ancien. L'étude s'est concentrée sur les séries 1 et 2 qui sont les plus anciennes et qui montrent une plus grande variété d'objets. L'attribution chronologique de la sépulture de Lothéa (Quimperlé, Finistère; fig. 15, n ${ }^{\text {os }} 22$, 30, 36, 37, 41 et 42) est discutable. J. Briard la considère comme tardive (Briard, 1984, p. 191) et S. Needham la rapporte à la série 2 (Needham, 2000, p. 158). L'attribution chronologique des vases funéraires de l'âge du Bronze ancien est rendue difficile par la rareté des mobiliers associés et une documentation lacunaire.

Plusieurs objets sont communs aux deux périodes qui nous intéressent: les pointes de flèches (fig. 15, $\mathrm{n}^{\mathrm{os}} 1$ à 3 et 21 à 27), les brassards d'archer (fig. 15, $\mathrm{n}^{\text {os }} 5,6$ et 30 à 33 ), les polissoirs en grès (fig. $15, \mathrm{n}^{\text {os }} 9$ et 35 ), les poignards (fig. $15, \mathrm{n}^{\text {os }} 11,12,36,37,53$ et 54 ) et les haches en métal (fig. 15, $n^{\text {os }} 13,38$ et 39), les pendeloques (fig. 15, n ${ }^{\text {os }} 14,15$ et 42 à 44), les gobelets en céramique (fig. $15, \mathrm{n}^{\text {os }} 16,17$ et 47 à 50 ) et les perles (fig. 15, $\mathrm{n}^{\mathrm{os}} 18,19,51$ et 52). De rares pointes en métal, pointe de Palmela en cuivre arsénié (Gandois, 2008; fig. $15, \mathrm{n}^{\circ} 3$ ) et pointes de flèches en alliage cuivreux (fig. 15, $\mathrm{n}^{\text {os }} 28$ et 29), se retrouvent dans le Campaniforme et l'âge du Bronze ancien, mais elles ne paraissent pas remplir la même fonction. Un nombre moins grand d'objets est exclusif à une période : l'outillage en silex (fig. $15, \mathrm{n}^{\text {os }} 7$ et 8 ), les poignards en silex du Grand-Pressigny (fig. 15, $\mathrm{n}^{\circ} 10$ ) et les boutons perforés en «V» (fig. 15, $\mathrm{n}^{\mathrm{O}} 18$ ) pour le Campaniforme; les aiguisoirs (fig. 15, no 34 ), les objets métalliques, tels que les épingles (fig. 15, $\mathrm{n}^{\circ} 40$ ), les chaînes (fig. 15, $\mathrm{n}^{\mathrm{O}} 41$ ), les gobelets précieux (fig. 15, $\mathrm{n}^{\text {os }} 45$ et 46) pour l'âge du Bronze ancien.

De ce fait, la plupart des types d'objets sont communs aux deux ensembles. Des objets sont typologiquement identiques : pendeloques trapézoïdales et discoïdes (fig. 15, $\mathrm{n}^{\text {os }} 14,15$ et 42 à 44), et brassards d'archer de taille fonctionnelle (fig. 15, $\mathrm{n}^{\text {os }} 5,6$ et 30 ). Il faut souligner le petit poignard de Coatjou-Glas (Plonéis, Finistère : fig. $15, \mathrm{n}^{\mathrm{O}} 12$ ) qui présente des traits chalcolithiques (soie développée) et des aspects de l'âge du Bronze ancien (trou de rivet, décoration avec un filet parallèle aux bords : fig. $15, \mathrm{n}^{\text {os }} 36,37$ et 54). Parallèlement, la sépulture du Moulin-deKergoniou (Guissény, Finistère ; archives Chatellier; Briard, 1984, p. 242) a livré un vase biconique à trois anses et un petit poignard qui montre une soie développée, un décor d'un seul filet et une absence de lunule à la garde (fig. 15, n 53). Là encore, l'aspect de prototype renvoie à une phase ancienne de l'âge du Bronze ancien. Le cas le plus spectaculaire est celui du gobelet en argent de Saint-Fiacre (Melrand, Morbihan). Dans une synthèse sur les gobelets précieux trouvés le long de la Manche, S. Needham a proposé une reconstitution des fragments de ce gobelet (Needham et al., 2006, p. 95). L'étude de ces fragments a montré qu'un morceau correspondait à un fond bombé, un deuxième à une partie de carène et deux autres à un bord. La forme reconstituée représente un gobelet en argent d'allure campaniforme (fig. 15, $n^{\circ} 45$ ). Ce gobelet en argent est associé à du mobilier métallique de l'âge du Bronze ancien (Aveneau de la Grancière, 1898). Pour la Bretagne, seuls trois de ces gobelets précieux sont connus, dont un dans la tombe Brun-Bras (SaintAdrien, Côtes-d'Armor; fig. 15, no 46). Tout semble concorder pour considérer le mobilier des dépôts funéraires des séries 1 et 2 comme une évolution du mobilier campaniforme.

On retrouve rarement, dans les sépultures des séries 1 et 2, de mobilier strictement identique à celui connu pour le Campaniforme. On peut tout de même citer les pointes de flèches, le brassard d'archer en schiste rouge et la pendeloque en roche verte du tumulus de Lothéa (Quimperlé, Finistère; fig. 15, $n^{\text {os }} 22,30$ et 42), qui ne dépareraient pas en contexte campaniforme. Les modalités de cette étroite filiation peuvent être précisées d'après la nature de ces objets. D'une part, il semble que les objets fonctionnels, tels que les polissoirs (fig. 15, $\mathrm{n}^{\mathrm{o}} 35$ ), les poignards (fig. $15, \mathrm{n}^{\text {os }} 36,37$ et 54 ) et certaines haches (fig. 15, no 39 ), auxquels on peut ajouter les aiguisoirs (fig. 15, $\mathrm{n}^{\mathrm{o}} 34$ ), connaissent un développement considérable de leurs dimensions. Le cas le plus emblématique est celui de la lame de Lothéa (Quimperlé, Finistère; fig. 15, no 37 ) qui mesure 53 centimètres de longueur, ce qui est sans commune mesure avec la majorité des poignards de type armoricain. D'autre part, des objets fonctionnels campaniformes entrent à l'âge du Bronze ancien dans une sphère 
plus symbolique. Brassards d'archer et gobelets sont «détournés de leur fonction première» (Pailler, 2007, p. 286) pour devenir des biens de prestige. Leurs dimensions sont réduites et des matières précieuses (or, argent, ambre, jais) sont employées pour la réalisation de gobelets précieux et de brassards d'archer non fonctionnels, appelés «bracer-ornament» par les Britanniques (Needham, 2000, p. 159; fig. 15, nn 31 à 33 ). Dans la même logique, les pointes de flèches de type armoricain illustrent le cas d'armatures qui sont le produit d'ateliers spécialisés. Les roches tenaces des pendeloques et des perles campaniformes (fig. 15, $\left.\mathrm{n}^{\text {os }} 14,15\right)$ sont remplacées par l'ambre de la Baltique (Gardin, 1989 et 1996; fig. 15, $\mathrm{n}^{\text {os }} 42,43$ et 51).

Le développement de la métallurgie à l'âge du Bronze ancien ne montre pas de rupture manifeste avec le Campaniforme, du moins dans les dépôts funéraires. On assiste plutôt à une continuité avec de petits changements (utilisation de nouvelles matières et évolution des formes). L'accumulation d'objets dans les tombes à l'âge du Bronze ancien intervient essentiellement avec la série 2, comme le montre S. Needham (2000, p. 160). Il semble que la série 1 corresponde à la mise en place de l'innovation métallurgique et la série 2 à l'accaparement de ce nouvel artisanat et des «biens précieux » (Salanova, 2007, p. 319) par une élite sociale qui se réfère à une tradition campaniforme. Il n'est pas anodin que brassards d'archer et pointes de flèches soient déposés conjointement dans les tombes de la série 2, comme à Kernonen (Plouvorn, Finistère; Briard, 1970a) et Kerguévarec (Plouyé, Finistère ; Lukis, 1884 ; Briard et al., 1994). La métallurgie du bronze exige l'utilisation étain, une ressource relativement rare en Europe que la Bretagne recèle en abondance (Guiges, 1970; Giot et Lulzac, 1998). En retour, cet atout économique pourrait, par des échanges, avoir donné accès à des matières précieuses, telles que le silex blond translucide ou l'ambre de la Baltique (Gardin, 1996), et à des produits finis, comme les perles en faïence (Briard, 1984, p. 151; fig. $15, \mathrm{n}^{\circ} 52$ ) ou les lunules en or irlandaises (dépôt de Kerivoa, Bourbriac, Côtes-d'Armor; Taylor, 1970). Une partie de ces objets sera accaparée par les élites et les accompagnera dans leurs tombes. L'autre partie semble avoir obtenu un statut particulier, mis à part les haches plates généralement découvertes hors contexte. Les rares dépôts non funéraires de l'âge du Bronze ancien sont constitués de haches plates irlandaises en cuivre arsénié (Briard, 1965), de lunules en or (Briard, 1984, p. 132), d'un gobelet précieux et d'une cuiller en or à Plumiliau (Côtes-d'Armor; Briard, 1984, p. 134) et d'une seconde cuiller en or à Plouarzel (Finistère; Briard, 1965).

La continuité de la culture matérielle entre Campaniforme et Bronze ancien pourrait s'expliquer par l'utilisation des symboles ancestraux afin d'asseoir une élite sociale émergente (Needham, 2000, p. 190) qui s'est accaparée la production hautement stratégique de l'étain et la technologie du bronze. La série 3 voit l'abandon progressif des références au Campaniforme, mais conserve les nouveaux symboles élaborés par les séries 1 et 2, à savoir la pointe de flèche, le poignard de type armoricain et la hache à légers rebords.

\section{La flèche, le poignard et le vase}

La relation entre les sépultures à pointes de flèches et les autres tombes de l'âge du Bronze ancien est difficile à cerner d'un point de vue chronologique. Une division entre tumulus à pointes de flèches et tumulus à poterie a été observée dès 1899 (Aveneau de la Grancière, 1899; Martin, 1900). Avec les première et deuxième séries, P.-R. Giot et J. Cogné expliquaient cette séparation de

Fig. 15 (à droite) - Relation entre les cultures matérielles du Campaniforme et de l'âge du Bronze ancien. Poignards et gobelets (échelle : 1:2) : $\mathrm{n}^{\text {os }} 1$ et 4 ) dolmen de Kercadoret, Locmariaquer, Morbihan (dessins C. Nicolas); $\mathrm{n}^{\text {os }} 2,6,7,12$ et 19) tumulus de Coatjou-Glas, Plonéis, Finistère (d'après Chatellier, archives et Chaigneau-Normand, 1994); $\mathrm{n}^{\text {os }} 3,8,13$ et 14) tumulus de Kermenhir, Poullan-sur-Mer, Finistère (d'après les archives du Chatellier) ; $\mathrm{n}^{\text {os }} 5,9,11,17$ et 18 ) allée couverte de Penker, Plozévet, Finistère (d'après les archives du Chatellier et Salanova, 2000); ${ }^{\circ}$ 10) allée couverte de Kerandrèze, Moëlan-sur-Mer, Finistère (d'après les archives du Chatellier); $n^{\circ} 15$ ) allée couverte de Kernic, Plouescat, Finistère (dessin C. Nicolas); ${ }^{\circ} 16$ ) chambre D, cairn de Barnenez, Plouzéoc'h, Finistère (d'après Giot, 1987); n 20) sépulture mégalithique en équerre de Goërem, Gâvres, Morbihan (d'après L'Helgouac'h, 1970); nos 21 et 23) tumulus de Cazin, Plouigneau, Finistère (dessin C. Nicolas); $n^{\text {os }} 22,30,36,37,41$ et 42) tumulus de Lothéa, Quimperlé, Finistère (d'après Taylor et al., 1846 et Briard et Mohen, 1974); $n^{\text {os }} 24,32,38$ et 39) tumulus de Kerguévarec, Plouyé, Finistère (dessin C. Nicolas et d'après Briard et al., 1994); $n^{\text {os }} 25,31,40,43$ et 44) tumulus de Kernonen, Plouvorn, Finistère (d'après Briard, 1970a); $\mathrm{n}^{\text {os }} 26,33$ et 34 ) tumulus de la Motta, Lannion, Côtes-d'Armor (d'après Butler et Waterbolt, 1974); n²7) sépulture de Limbabu, SaintThégonnec, Finistère (dessin C. Nicolas); $n^{\text {os }} 28,29$ et 45 ) tumulus de Saint-Fiacre, Melrand, Morbihan (d'après Aveneau de la Grancière 1898 et Needham et al., 2006); $\mathrm{n}^{\circ} 35$ ) tumulus de Mouden-Bras, Pleudaniel, Côtes-d'Armor (d'après Balquet, 2001); $\mathrm{n}^{\circ} 46$ ) tumulus de Brun-Bras, Saint-Adrien, Côtes-d'Armor (d'après Briard, 1984); $n^{\circ} 47$ ) tumulus de Bignat, Kerien, Côtes-d'Armor (d'après Briard, 1965); n 48) tumulus de Kerbran, La Feuillée, Finistère (d'après Briard et al., 1994); nº 49) coffre de Juno-Bella 2, Berrien, Finistère (d'après Briard et al., 1994); $\mathrm{n}^{\circ} 50$ ) coffre de Gouerven, Lesneven, Finistère (d'après Briard, 1966); $\mathrm{n}^{\circ}$ 51) tumulus de Kerstrobrel, Crozon, Finistère (d'après Gardin, 1996); $\mathrm{n}^{\mathrm{o}} 52$ ) tumulus de Mez-Nabat, Plouhinec, Finistère (d'après Briard, 1984); no 53) tumulus du Moulin-de-Kergoniou, Guissény, Finistère (d'après les archives du Chatellier); $\mathrm{n}^{\circ}{ }^{54}$ ) tumulus de Sainte-Anne, Goudelin, Finistère (d'après Stevenin, 2000).

Fig. 15 (right) - Links between Bell Beaker and Early Bronze age material cultures. Daggers and beakers (scale: 1:2): 1 and 4) Kercadoret dolmen, Locmariaquer, Morbihan (drawings C. Nicolas); 2, 6, 7, 12 and 19) Coatjou-Glas barrow, Plonéis, Finistère (after Chatellier, archives and Chaigneau-Normand, 1994); 3, 8, 13 and 14) Kermenhir barrow, Poullan-sur-Mer, Finistère (after Chatellier, archives); 5, 9, 11, 17 and 18) Penker gallery grave, Plozévet, Finistère (after Chatellier, archives and Salanova, 2000); 10) Kerandrèze gallery grave, Moëlan-sur-Mer, Finistère (after Chatellier, archives); 15) Kernic gallery grave, Plouescat, Finistère (drawing C. Nicolas); 16) chamber D, Barnenez cairn, Plouzéoc'h, Finistère (after Giot, 1987); 20) Goërem squared gallery grave, Gâvres, Morbihan (after L'Helgouac'h, 1970); 11 and 23) Cazin barrow, Plouigneau, Finistère (drawings C. Nicolas); 22, 30, 36, 37, 41 and 42) Lothéa barrow, Quimperlé, Finistère (after Taylor et al., 1846 and Briard and Mohen, 1974); 24, 32, 38 and 39) Kerguévarec barrow, Plouyé, Finistère (drawing C. Nicolas and after Briard et al., 1994); 25 , 31, 40, 43 and 44) Kernonen barrow, Plouvorn, Finistère (after Briard, 1970a); 26, 33 and 34) la Motta barrow, Lannion, Côtes-d'Armor (after Butler and Waterbolt, 1974); 27: Limbabu grave, Saint-Thégonnec, Finistère (drawing C. Nicolas); 28, 29 and 45) Saint-Fiacre barrow, Melrand, Morbihan (after Aveneau de la Grancière, 1898 and Needham et al., 2006); 35) Mouden-Bras barrow, Pleudaniel, Côtes-d'Armor (after Balquet, 2001); 46) Brun-Bras barrow, Saint-Adrien, Côtes-d'Armor (after Briard, 1984); 47) Bignat barrow, Kerien, Côtes-d'Armor (after Briard, 1965); 48) Kerbran barrow, La Feuillée, Finistère (after Briard et al., 1994); 49) Juno-Bella 2 grave, Berrien, Finistère (after Briard et al., 1994); 50) Gouerven grave, Lesneven, Finistère (after Briard, 1966); 51) Kerstrobrel barrow, Crozon, Finistère (after Gardin, 1996); 52) MezNabat barrow, Plouhinec, Finistère (after Briard, 1984); 53) Moulin-deKergoniou barrow, Guissény, Finistère (after Chatellier, archives); 54) Sainte-Anne barrow, Goudelin, Finistère (after Stevenin, 2000). 


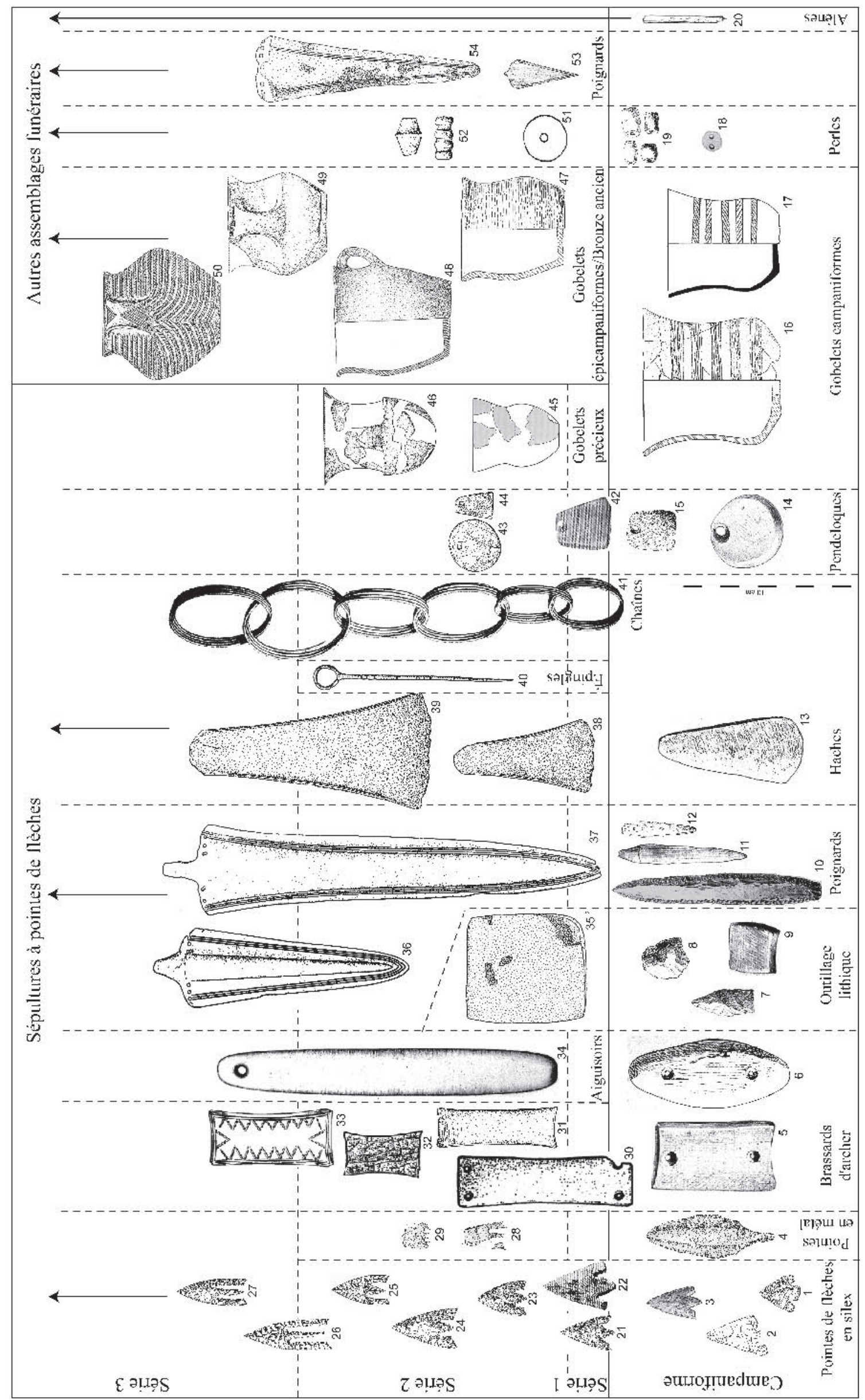



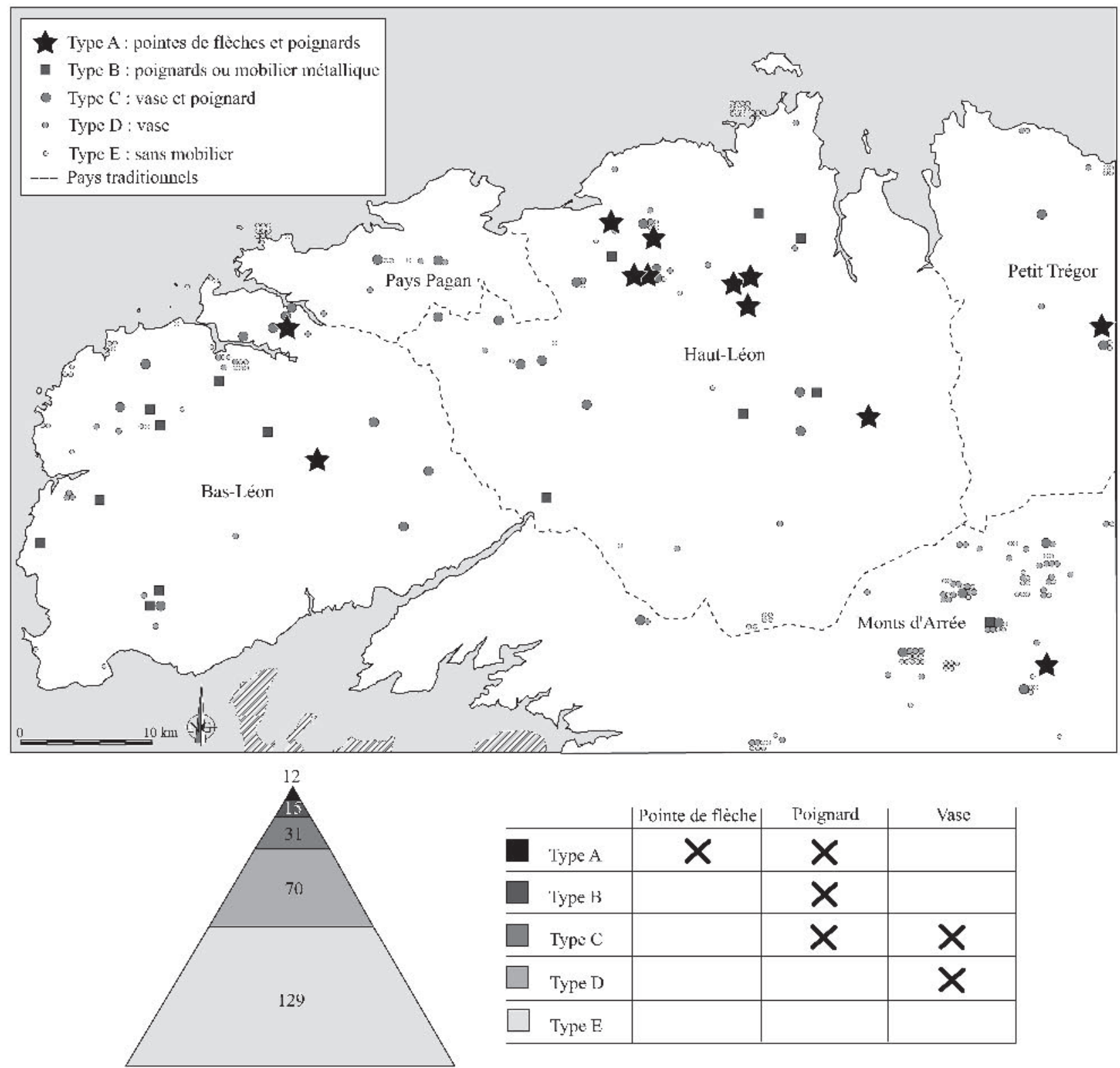

\begin{tabular}{l|c|c|c|} 
& Pointe de flèche & Toignard & Vase \\
\hline$\square$ Type A & $\boldsymbol{X}$ & $\mathbf{X}$ & \\
\hline$\square$ Type B & & $\mathbf{X}$ & \\
\hline$\square \quad$ Type C & & $\mathbf{X}$ & $\mathbf{X}$ \\
\hline$\square \quad$ Type D & & & $\mathbf{X}$ \\
\hline$\square \quad$ Type E & & & \\
\hline
\end{tabular}

Fig. 16 - Types de dépôts funéraires de l'âge du Bronze ancien et leur répartition dans le nord du Finistère (fond de carte A. Leroy). Fig. 16 - Types of Early Bronze Age grave goods and their distribution in Northern Finistère (background map A. Leroy).

façon chronologique puisqu'ils ne pouvaient conclure à une différenciation sexuelle (Giot et Cogné, 1951, p. 436). S. Needham a proposé de scinder en deux la série des tumulus à poterie en distinguant les tombes à poteries, contemporaines des tumulus à pointes de flèches, et les sépultures à vases et poignards, qui leur seraient postérieures. Cette série à vases et poignards se caractériserait par des poignards en bronze de type Bourbriac, qui apparaissent comme récents (Needham, 2000, p. 152-158). Elle est basée sur un corpus partiel de 15 sépultures pour l'ensemble de la Basse-Bretagne, alors que 31 tombes à vases et poignards sont connues dans le nord du Finistère (fig. 16). Certaines attributions typologiques peuvent être contestées : le poignard de la sépulture de Sainte-Anne en Goudelin (Côtes-d'Armor) pourrait être tout aussi bien de type Rumédon que de type Bourbriac (fig. 15, n 54). La tombe du Moulin-de-
Kergoniou (Guissény, Finistère : fig. 15, no 53) montre l'association d'un vase et d'un petit poignard à soie plus proche des poignards chalcolithiques que des poignards de type Bourbriac.

Il n'y a aucun argument chronologique qui permette à l'heure actuelle de séparer les sépultures à pointes de flèches des sépultures à vases. Le paradigme posé au début des années 1950 a peu à peu été nuancé, puis remis en cause par les datations radiocarbone (Briard, 1984, p. 192) et le mobilier archéologique (Needham, 2000, p. 152). Les première et deuxième séries ne semblent pas représenter toute la variété des dépôts funéraires. On peut retenir que les assemblages funéraires de l'âge du Bronze ancien sont composés de mobiliers récurrents : la pointe de flèche, le poignard et le vase. Une description de la constitution des assemblages funéraires permet de retenir cinq grands types de dépôts : type A avec 
pointe de flèche et poignard, type $\mathrm{B}$ avec poignard ou mobilier métallique, type $\mathrm{C}$ avec vase et poignard, type $\mathrm{D}$ avec vase, type E sans mobilier (fig. 16). Les tombes sans mobilier sont attribuables à l'âge du Bronze ancien par leurs structures funéraires (Lecerf, 1979; Briard, 1984), mais on ne peut exclure que quelques sépultures soient un peu plus anciennes ou plus récentes.

Dans le nord du Finistère, nous avons réalisé un inventaire de 257 sépultures fouillées dont la composition du dépôt funéraire est assurée. Le vase est attesté dans 101 tombes. Le poignard en métal est relativement fréquent, on le trouve dans 58 sépultures. Seules 12 sépultures sur 257 contiennent des pointes de flèches (fig. 16). Le cas des tumulus des monts d'Arrée montre une répartition sans doute assez proche de la réalité. Entre 1895 et 1896, P. du Chatellier fouilla plus d'une centaine de monuments funéraires dans les monts d'Arrée (Chatellier, 1897), ce qui a permis de documenter le contenu des dépôts funéraires de façon systématique, offrant un échantillon représentatif de la réalité de l'âge du Bronze ancien. Cela pourrait expliquer le grand nombre de sépultures des types D et E dans le secteur (fig. 16).

Soixante-dix sépultures livrent un vase et 129 sépultures n'ont aucun mobilier. Trente et une tombes contiennent un ou deux poignards accompagnés d'un vase, 15 possèdent seulement du mobilier métallique et 12 contiennent des pointes de flèches et des poignards (fig. 16). Les types D et E forment une classe nombreuse possédant un vase ou pas de mobilier. Les types $\mathrm{B}$ et $\mathrm{C}$ constituent une classe plus réduite, dont le statut est souligné par le poignard. Le type A met en évidence une classe sociale restreinte qui s'exprime par la possession de pointes de flèches. Cette distribution des dépôts funéraires paraît refléter une société fortement hiérarchisée. Leur répartition spatiale semble confirmer cette analyse (fig. 16). Les sépultures à pointes de flèches paraissent être au centre de territoires correspondant peu ou prou aux pays traditionnels du nord du Finistère (Nicolas, 2008). Les autres types de dépôts funéraires se situent plutôt à la périphérie. À proximité des côtes actuelles et aux marges de ces probables territoires se trouvent des coffres qui ne livrent aucun mobilier caractéristique (fig. 16). La pointe de flèche apparaît bien comme l'insigne d'une élite sociale implantée au centre de territoires cohérents. Pour le Campaniforme, L. Salanova (2007) souligne le statut particulier des tombes à poignard en cuivre. Pendant l'âge du Bronze ancien en BasseBretagne, on constate une plus grande diffusion des poignards en bronze. Le rôle discriminant du poignard en cuivre campaniforme paraît transféré aux pointes de flèches de l'âge du Bronze ancien.

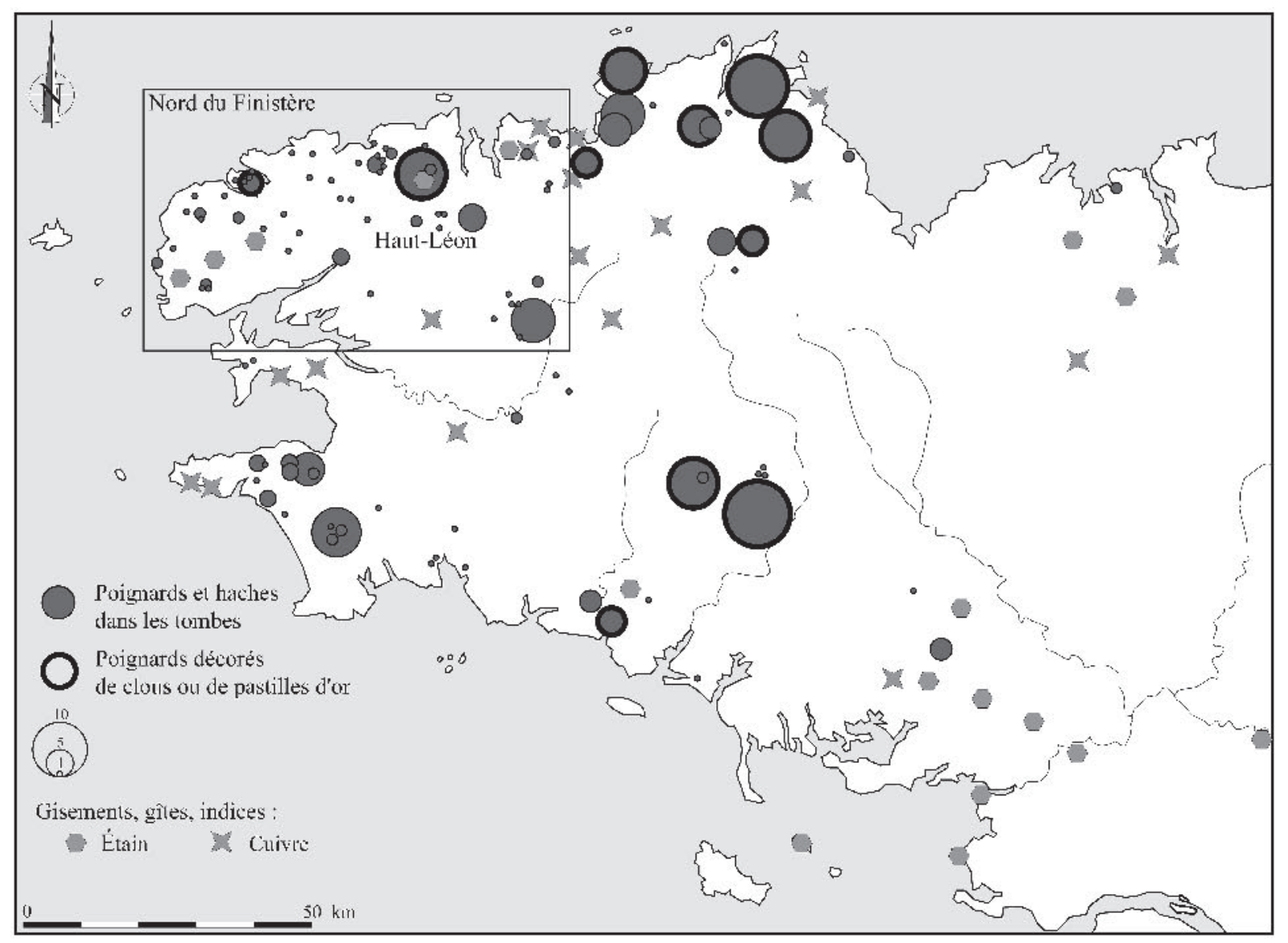

Fig. 17 - Répartition des poignards et des haches en bronze dans les tombes de l'âge du Bronze ancien en Bretagne et répartition des gisements métallifères (gisements métallifères d'après http://www.brgm.fr et Brun, 2003).

Fig. 17 - Distribution of bronze daggers and axes in the Early Bronze Age graves in Brittany and ore deposits (ore deposits after http://www.brgm. fr and Brun, 2003). 


\section{Le Haut-Léon au cour du Bronze ancien armoricain?}

La production léonarde de pointes de flèches de type Limbabu paraît inégalée dans le reste de la Bretagne. Elles proviennent d'ateliers de taille spécialisés dont les savoir-faire ont été peu à peu développés afin d'obtenir des pointes allongées aux ailerons très longs. Un tel savoir-faire implique probablement l'entretien d'artisans par une élite sociale. L'emploi exclusif du silex blond translucide exogène pour ce type d'armatures suppose des personnes capables d'entretenir des réseaux d'échange à longue distance. Cette maîtrise est d'autant plus importante que les pointes de type armoricain paraissent être le symbole et la légitimité du pouvoir des élites sociales.

Productions céramiques et métalliques tendent à confirmer le rôle moteur du Haut-Léon à l'âge du Bronze ancien de Basse-Bretagne. Dans le cadre d'un travail sur les vases des tumulus armoricains, C. Stevenin (2000) identifie deux productions dans le HautLéon, dont une de gobelets à parois fines décorés ou non de chevrons. Du point de vue de la métallurgie, la situation paraît moins évidente, mais on peut souligner que le Haut-Léon se situe au carrefour de deux pays riches en ressources métallifères. À l'ouest, le BasLéon recèle une des plus importantes ressources en étain d'Europe, exploitée au moins dès l'âge du Bronze moyen-final et encore à l'époque contemporaine (Giot et Lulzac, 1998). Au sud, les monts d'Arrée possèdent des ressources en cuivre mal connues, gisements peutêtre totalement épuisés suite à la forte activité métallurgique durant l'âge du Bronze en Bretagne (Kerforne, 1918). Rappelons que l'exploitation ancienne du cuivre breton a longtemps été jugée insignifiante en raison de la faible taille ou de la profondeur des gisements (Briard, 1984, p. 94). Toutefois on peut suggérer que de petites ressources en cuivre ont pu suffire à l'élaboration des 40 haches et 187 poignards en bronze découverts dans les tombes de l'âge du Bronze ancien (Briard, 1984; Nicolas, 2008). Il reste que le nord du Finistère est le seul secteur à présenter les ressources en étain - et peut-être aussi en cuivre - nécessaires à l'élaboration du bronze, et de grandes concentrations de tumulus (fig. 16 et 17). La nature des dépôts funéraires atteste une grande diffusion du poignard en bronze dans le Léon. Ainsi, 58 tombes ont livré au moins un poignard en bronze dans le nord du Finistère, soit autant que dans le reste de la Bretagne (53 tombes). Nous observons une dichotomie entre le reste de la Bretagne, où le métal paraît thésaurisé avec un faible nombre de sépultures livrant un grand nombre d'objets métalliques, certains décorés de clous ou de pastilles d'or, et le nord du Finistère, où le métal semble avoir une plus grande diffusion avec un grand nombre de sépultures livrant un ou deux poignards en bronze (fig. 17). Cette dichotomie suggère que le nord du Finistère et au premier titre le Haut-Léon jouent un rôle prépondérant dans la production métallurgique (fig. 17). Vases, pointes de flèches et poignards montrent le rôle de première importance du Haut-Léon dans la production de biens socialement valorisés. Le brassard et les 11 pendeloques en ambre de la Baltique retrouvés dans la tombe de Kernonen (Plouvorn, Finistère; Briard, 1970a; fig. 15, $\mathrm{n}^{\text {os }} 31,43$ et 44 et fig. 17) soulignent ce rôle moteur et la capacité d'entretenir des réseaux à longue distance.

\section{À LA RECHERCHE DE L'ÂGE DU BRONZE ANCIEN}

Les observations sur l'importance du Haut-Léon permettent d'orienter les recherches sur un âge du Bronze ancien encore largement méconnu en dehors du monde funéraire. Plusieurs sites sont connus le long des côtes grâce à l'érosion marine, comme sur la plage du Lividic (Plonéour-Trez, Finistère; Giot et al., 1995). Les structures d'habitats restent exceptionnelles : cabane de la Grosse-Roche (Saint-Jacut-de-la-Mer, Côtes-d'Armor; Briard et al., 1990), maison en pierres sèches de Beg-ar-Loued (île Molène, Finistère; Pailler et al., 2010). De rares prospections dans l'intérieur des terres ont livré des séries de mobiliers rattachables à l'âge du Bronze ancien, comme sur le plateau du Collédic à Saint-Nicolas-du-Pélem (Côtes-d'Armor; Le Provost et al., 1972). Le rôle majeur du Haut-Léon dans la production de biens socialement valorisés et l'existence de réseaux à longue distance permettent d'espérer une concentration des vestiges et une plus grande visibilité archéologique. Concentrer les recherches de terrain sur le Haut-Léon permettrait sans doute de mieux percevoir les sites domestiques ou cérémoniels de l'âge du Bronze ancien ainsi que le rôle de la Basse-Bretagne dans la production d'armatures de prestige et le développement de la métallurgie du bronze.

Remerciements : Cette étude n'aurait jamais pu être réalisée sans la bienveillance des conservateurs de plusieurs institutions : J.-L. Monnier (UMR 6566; musée de Préhistoire finistérienne, Penmarc'h), A. Villes (musée d'Archéologie nationale, Saint-Germainen-Laye), M. Le Goffic (service départemental d'Archéologie du Finistère), P. Jourdan (musée des Jacobins, Morlaix). Je tiens à remercier J. Pelegrin (UMR 7055), S. Blanchet (INRAP Grand-Ouest), $\mathrm{Ph}$. Forré (INRAP Grand-Ouest) et E. Ghesquière (INRAP Grand-Ouest) pour leurs lumières sur la technologie lithique et les matières premières, et $\mathrm{C}$. Le Carlier (UMR 6566) pour son avis sur la question du cuivre en Bretagne. Il m'est agréable de remercier Y. Sparfel pour ses nombreuses informations sur des tumulus inédits. Enfin, un grand merci à F. Giligny, Y. Pailler, L. Manolakakis, membres de l'UMR 7041, et P. Gouletquer qui ont suivi de près l'aboutissement de ce travail et qui se sont proposés pour relire le manuscrit.

\section{NOTE}

(1) Collection Paul du Chatellier, série 100J, archives départementales du Finistère, Quimper. 


\section{RÉFÉRENCES BIBLIOGRAPHIQUES}

AVENEAU DE LA GRANCIÈRE P. (1898) - Le Bronze dans le centre de la Bretagne-Armorique : fouille du tumulus à enceinte semicirculaire de Saint-Fiacre en Melrand, canton de Baud, Bulletin de la Société polymathique du Morbihan, 17, p. 81-95.

AVENEAU DE LA GRANCIÈRE P. (1899) - Le Bronze en BretagneArmorique : de quelques sépultures de l'époque du Bronze en Armorique occidentale, Bulletin archéologique de l'Association bretonne, 17 , p. 202-217.

BAILLY M. (2002) - La flèche et l'éclat : production et consommation des outillages lithiques taillés de la fin du Néolithique au début de l'âge du Bronze entre Saône et Rhône, 2600-2000 av. J.-C., Thèse de doctorat, Université de Franche-Comté, Besançon, 2 vol., 354 p.

BALQUET A. (1999) - Les débuts de la métallurgie et la civilisation des tumulus armoricains, Thèse de doctorat, Université Rennes 1 , Rennes, 3 vol., 730 p.

BALQUET A. (2001) - Les tumulus armoricains du Bronze ancien, Rennes, Ed. Institut culturel de Bretagne-Association des travaux du Laboratoire d'anthropologie et de préhistoire de l'université de Rennes 1 (Patrimoine archéologique de Bretagne), $150 \mathrm{p}$.

BENARD C., dit LE PONTOIS (1929) - Le Finistère préhistorique, Paris, Éd. Nourry (Publications de l'Institut international d'anthropologie 3), $337 \mathrm{p}$.

BLANCHET S. (2005) - La tombe du Bronze ancien de Crec'h-Perros à Perros-Guirec (22) : les premiers résultats, Bulletin de l'Association pour la promotion des recherches sur l'âge du Bronze, 2, p. 27-29.

BORDES F. (1947) - Étude comparative des différentes techniques de taille du silex et autres roches dures, L'anthropologie, 51, p. 1-29.

BRIARD J. (1965) - Les dépôts bretons et l'âge du Bronze atlantique, Rennes, Éd. Laboratoire d'anthropologie préhistorique de la faculté des sciences de Rennes (Travaux), $352 \mathrm{p}$

BRIARD J. (1966) - Découverte d'une tombe de l'âge du Bronze à Gouerven en Lesneven (Finistère), Annales de Bretagne, 63, p. 7-12.

BRIARD J. (1968) - Un tumulus du Bronze Ancien à Lescongar en Plouhinec (Finistère), Gallia Préhistoire, 11, p. 247-259.

BRIARD J. (1970a) - Un tumulus du Bronze ancien : Kernonen en Plouvorn (Finistère), L'anthropologie, 74, p. 5-56.

BRIARD J. (1970b) - Les tumulus de l'âge du Bronze de PlouvornPlouzévédé (Finistère), Bulletin de la Société préhistorique française, 67, Étude et travaux 1, p. 372-385.

BRIARD J. (1972) - Les tumulus de Kervini en Poullan : fouilles de 1971, Bulletin de la Société archéologique du Finistère, 98, p. 21 38

BRIARD J. (1976) - Acculturations néolithiques et campaniformes dans les tumulus armoricains, in J. de Laet dir., Acculturations and Continuity in Atlantic Europe Mainly During the Neolithic Period and the Bronze Age, Proceedings of the $4^{\text {th }}$ Atlantic Colloquium, Ghent, 1975 , Brugge, De Tempel (Dissertationes archaeological Gandenses 16), p. 34-44.

BRIARD J. (1977a) - Rapport scientifique sur la fouille de sauvetage du tumulus de Cazin, à Plouigneau (Finistère), Rennes, Service régional de l'Archéologie de Bretagne (Rapport scientifique 711) $29 \mathrm{p}$.

BRIARD J. (1977b) - Mégalithes et tumulus à l'âge du Bronze. La «Dame de Kersandy », Plouhinec (Finistère), in L'architecture mégalithique, Actes du colloque du $150^{\mathrm{e}}$ anniversaire de la Société polymathique du Morbihan, Vannes, 1976, Vannes, Éd. Château-Gaillard, p. $31-48$.

BRIARD J. (1978) - Das Silbergefäss von Saint-Adrien, Côtes-du-Nord, Archäologisches Korrespondenzblatt, 8, p. 13-20.

BRIARD J. (1984) - Les tumulus d'Armorique, Paris, Picard (L'âge du Bronze en France 3), 304 p.
BRIARD J. (2005) - Des sépultures à inhumations du Bronze ancien aux sépultures à incinérations du Bronze final en Armorique, in C. Mordant et G. Depierre dir., Les pratiques funéraires à l'âge du Bronze en France, Actes de la table ronde de Sens (Yonne), 1998, Paris-Sens, Éd. CTHS (Documents préhistoriques 19)-Société archéologique de Sens, p. 243-255.

BRIARD J., CABILLIC A., MARGUET A., ONNÉE Y. (1982) - Les fouilles de Kersandy à Plouhinec (Finistère) : une tombe du Bronze ancien à «déesse-mère» néolithique, Bulletin de la Société archéologique du Finistère, 110, p. 17-39.

BRIARD J., GEBHARDT A., MARGUERIE D., NICOLARDOT J.-P., ROBINO P. (1990) - Habitats et environnement de l'âge du Bronze en Bretagne, in M.-J. Roulière-Lambert et M. Oberkampf dir. Un monde villageois : habitat et milieu naturel en Europe de 2000 à 500 av. J.-C., Lons-le-Saunier, Éd. Cercle Girardot, p. 37-44.

BRIARD J., GIOT P.-R. (1956) - Typologie et chronologie du Bronze ancien et du Premier Bronze moyen en Bretagne, Bulletin de la Société préhistorique française, 53, 7-8, p. 363-373.

BRIARD J., LE GOFFIC M., ONNEE Y. (1994) - Les tumulus de l'âge du Bronze des monts d'Arrée, Rennes, Éd. Institut culturel de Bretagne-Association des travaux du Laboratoire d'anthropologie et de préhistoire de l'université de Renne 1 (Patrimoine archéologique de Bretagne), 96 p.

BRIARD J., L'HELGOUACH J. (1957) - Chalcolithique, Néolithique secondaire, survivances néolithiques à l'âge du Bronze ancien en Armorique, Rennes, Éd. Laboratoire d'Anthropologie préhistorique de la faculté des sciences de Rennes (Travaux), $72 \mathrm{p}$.

BRIARD J., MOHEN J.-P. (1974) - Le tumulus de la forêt de Carnoët à Quimperlé (Finistère), Antiquités nationales, 6, p. 46-60.

BRUN P. (2003) - La signification variable des dépôts funéraires et non funéraires de l'âge du Bronze, in J. Bourgeois, I. Bourgeois et B. Cheretté dir., Bronze Age and Iron Age Communities in North-Western Europe, Handelingen van het Contactforum «Exchange and Interaction: the Role of the Scheldt and Meuse During the Bronze Age and Iron Age», 2002, Bruxelles, Éd. Koninklijke Vlaamse Academie van Belgie voor Wetenschappen en Kunsten, p. 61-73.

BUTLER J.-J., WATERBOLT H. (1974) - La fouille de A.-E. Van Giffen à la Motta, un tumulus de l'âge du Bronze ancien à Lannion (Bretagne), Palaeohistoria, 16, p. 107-167.

CHAIGNEAU-NORMAND M. (1994) - Les pointes de flèches en silex des tumulus armoricains, Mémoire de maîtrise, Université Rennes 2 , Renne, 5 vol, 1116 p.

CHATELLIER P. du (1880a) - Exploration du tumulus du Kerhué-Bras en Plonéour-Lanvern, Matériaux pour l'histoire primitive et naturelle de l'homme, 11, p. 289-298.

CHATELLIER P. du (1880b) - Exploration du tumulus du Kerhué-Bras en Plonéour-Lanvern (Finistère), Revue archéologique, 39, p. 312 .

CHATELLIER P. du (1887) - Sépulture de Coatjou-Glas en Plonéis (Finistère), Matériaux pour l'histoire primitive et naturelle de l'homme, 15, p. 49-52.

CHATELLIER P. du (1897) - Exploration sur les montagnes d'Arrhées et leurs ramifications, années 1895-1896, Bulletin de la Société d'émulation des Côtes-du-Nord, 25, p. 51-112.

CHATELLIER P. du (1898a) - Exploration des tumulus du Fao-Youen et de Cosmaner en Plonéour-Lanvern (Finistère), Bulletin de la Société archéologique du Finistère, 25, p. 128-133.

CHATELLIER P. du (1898b) - Fouille d'un tumulus à Kergournadec, Finistère, en 1638, Bulletin de la Société archéologique du Finistère, 25 , p. $321-322$

CHATELLIER P. du (1907) - Les époques préhistoriques et gauloises dans le Finistère : inventaire des monuments de ce département des temps préhistoriques à la fin de l'occupation romaine, $2^{\mathrm{e}}$ édition revue 
et augmentée, Renne, Plihon et Hommay (Études archéologiques sur le Finistère), 391 p.

CLOSMADEUC G. de (1864) - Dolmen tumulaire de Crubelz (commune de Belz, arrondissement de Lorient), Revue archéologique, 9, p. 393-403.

COATIVY Y. dir. (2006) - Paul du Chatellier, collectionneur finistérien (1833-1911) : archives de Kernuz, sous-série 100 j 1249 à 1252, Brest, Éd. Association finistérienne pour l'université du Temps libre-Centre de recherche bretonne et celtique, $473 \mathrm{p}$.

ÉLUÈRE C. (1982) - Les ors préhistoriques, Paris, Picard (L’âge du Bronze en France 2), 287 p.

FREMINVILLE chevalier de, DE LA POIX C.-P. (1832-1835) - Antiquités de la Bretagne : le Finistère, Brest, Impr. Le Fournier et Deperiers.

FURESTIER R. (2005) - Les industries lithiques campaniformes du sud-est de la France, Thèse de doctorat, Université de Provence-AixMarseille 1, Aix-en-Provence, 3 vol., 632 p.

GAILLARD F. (1892) - Inventaire des monuments mégalithiques du Morbihan dans le périmètre des acquisitions de l'État, Revue des sciences naturelles de l'Ouest, 2, p. 175-191.

GALLAY G. (1981) - Die kupfer- und altbronzezeitlichen Dolche und Stabdolche in Franckreich, Munich, Éd. Beck (Prähistorische Bronzefunde VI, 5), $164 \mathrm{p}$.

GANDOIS H. (2008) - L'apparition du bronze à l'étain dans le nord-ouest de la France, Mémoire de master 1, Université Paris 1, Paris, 140 p.

GARDIN (du) C. (1989) - Les perles d' «ambre» bretonnes : ambre balte ou résine fossile locale?, Actes de la Journée préhistorique et protohistorique de Bretagne, 1989, Rennes, Ed. DRAC BretagneLaboratoire d'anthropologie et d'archéométrie de l'université Rennes 1, p. 38-40

GARDIN (du) C. (1996) - L'ambre en France au Bronze ancien : données nouvelles, in C. Mordant et O. Gaiffe dir., Cultures et sociétés du Bronze ancien en Europe, Actes du $117^{\mathrm{e}}$ congrès national des Sociétés historiques et scientifiques, Clermont-Ferrand, 1992, Paris, Éd. CTHS, p. 189-195.

GIOT P.-R. (1953) - Quelques tombes finistériennes de l'âge du Bronze découvertes fortuitement, Bulletin de la Société archéologique du Finistère, 79, p. 31-42.

GIOT P.-R. (1977) - Chronique de préhistoire et de protohistoire finistériennes pour 1977, Bulletin de la Société archéologique du Finistère, 105 , p. 12.

GIOT P.-R. (1987) - Barnenez, Carn, Guennoc, Rennes, Éd. Laboratoire d'anthropologie, de préhistoire, de protohistoire et du Quaternaire armoricain de l'université Rennes 1 (Travaux), 2 vol., 232 p.

GIOT P.-R. (1988) - Chronique de préhistoire et de protohistoire finistériennes et des archéosciences pour 1988, Bulletin de la Société archéologique du Finistère, 117, p. 9-27.

GIOT P.-R. (1997) - Paul du Chatellier, sa famille, sa vie, son œuvre, sa collection, Antiquités nationales, 29, p. 35-44.

GIOT P.-R., BRIARD J., PAPE L. (1995) - Protohistoire de la Bretagne, $2^{\mathrm{e}}$ édition, Rennes, Éd. Ouest-France, 422 p.

GIOT P.-R., COGNE J. (1951) - L'âge du Bronze ancien en Bretagne, L'anthropologie, 55, 5-6, p. 424-444.

GIOT P.-R., L'HELGOUACH J., BRIARD J. (1962) - La Bretagne : préhistoire et protohistoire, Grenoble, Éd. Arthaud, 297 p.

GIOT P.-R., LULZAC Y. (1998) - Datation à l'âge du Bronze d'une exploitation de cassitérite dans le Finistère, Bulletin de la Société préhistorique française, 95, p. 598-600.

GUIGES J. (1970) - Les minéraux alluvionnaires de Basse-Bretagne, Pen-ar-Bed, 7, 60, p. 239-248.

GUYODO J.-N. (2001) - Les assemblages lithiques des groupes néolithiques sur le Massif armoricain et ses marges, Thèse de doctorat, Université Rennes 1, Rennes, 331 p.
HALNA DU FRETAY M.C.M. (1888) - Bronzes et silex réunis dans les deux tumulus accolés de Kervini en Poullan (Finistère), Bulletin archéologique de l'Association bretonne, 8, p. 186-192.

HALNA DU FRETAY M.C.M. (1889) - Les âges préhistoriques et le début de l'ère chrétienne : incinérations et inhumations dans le Finistère et l'ouest de la Bretagne, Bulletin de la Société archéologique du Finistère, 16, p. 319-368.

HALNA DU FRETAY M.C.M. (1893) - Preuves à l'appui de l'histoire des temps néolithiques (deuxième partie), Bulletin de la Société archéologique du Finistère, 20, p. 138-140.

HUARD J. (1970) - Étude anatomique de quelques fragments de bois trouvés dans la tombe de Kernonen en Plouvorn (Finistère, France), in J. Briard, Un tumulus du Bronze ancien : Kernonen en Plouvorn (Finistère), L'anthropologie, 74, p. 50-56.

INIZAN M-L., REDURON M., ROCHE H., TIXIER J. (1995) - Préhistoire de la pierre taillée, t. 4 Technologie de la pierre taillée, Meudon, Éd. CREP, 199 p.

KERDREL de comte (1898) - Fouille d'un tumulus situé dans les bois de Keruzoret (Plouvorn), Bulletin de la Société archéologique du Finistère, 25, p. 23-24.

KERFORNE (1918) - Les ressources du sous-sol armoricain, Rennes, Éd. Oberthur, 39 p.

LECERF Y. (1979) - Les sépultures en coffres de l'âge du Bronze en Armorique, Mémoire de l'École pratique des hautes études, Paris, $92 \mathrm{p}$.

LE GOFFIC M. (1989) - Fouille d'une sépulture de la nécropole de l'âge du bronze de Graeoc en Saint-Vougay, Bulletin de la Société archéologique du Finistère, 118, p. 17-34.

LE GOFFIC M., NALLIER R. (2008) - Fouille d'un caveau du Bronze ancien à Lannilis (Finistère), Actes de la Journée du CreAAH : Archéologie, Archéosciences, Histoire, Rennes, Rennes, Éd. Université de Rennes 1, p. 41-43.

LE PONTOIS L. (1890) - Le tumulus de Cruguel en Guidel, Revue archéologique, 16, p. 304-338.

LE PROVOST F., GIOT P.-R., ONNÉE Y. (1972) - Prospections sur les collines de Saint-Nicolas-du-Pélem (Côtes d'Armor) du Chalcolithique à la Protohistoire, Annales de Bretagne, 79, p. 36-48.

LE ROUX C.-T. (1983) - Informations archéologiques de la circonscription de Bretagne, Gallia Préhistoire, 26, p. 309-333.

LE ROUZIC Z. (1933) - Morphologie et chronologie des sépultures préhistoriques du Morbihan, L'anthropologie, 43, p. 225-265.

L'HELGOUAC'H J. (1970) - Le monument mégalithique du Goërem à Gâvres (Morbihan), Gallia Préhistoire, 13, p. 217-261.

L'HELGOUAC'H J. (2001) - Le cadre culturel du campaniforme armoricain, in F. Nicolis dir., Bell Beakers Today: Pottery, People, Culture, Symbols in Prehistoric Europe, Proceedings of the International Colloquium, Trento, 1998, Trente, Éd. Provincia Autonoma di Trento-Servizio Beni Culturale et Uffico Beni Archeologici, p. 289-299.

LUKIS J. W. (1884) - Exploration d'un tumulus de l'époque du bronze dans la commune de Plouyé (Finistère), Matériaux pour l'histoire primitive et naturelle de l'Homme, $3^{\mathrm{e}}$ série, 1, p. 447-450.

MARCIGNY C., GHESQUIÈRE E. dir. (2003) - L'île de Tatihou (Manche) à l'âge du Bronze : habitats et occupation du sol, Paris, Éd. MSH (Documents d'archéologie française 96), 185 p.

MARSILLE L. (1913) - Le tumulus de Coët-er-Garf en Elven et les sépultures de l'âge du Bronze dans le Morbihan, Bulletin de la Société polymathique du Morbihan, 55, p. 110-121.

MARTIN A. (1900) - Les sépultures à belles pointes de flèches en silex, L'anthropologie, 11, p. 159-178.

MARTIN A. (1904a) - La fouille de Tossen-Rugouec en Prat, Bulletin de la Société archéologique du Finistère, 31, p. 3-17. 
MARTIN A. (1904b) - Fouille du tumulus du Rumédon en Ploumiliau, Côtes-du-Nord, Bulletin de la Société archéologique du Finistère, 31 , p. $128-154$.

MARTIN A., BERTHELOT DU CHESNAY C. (1899) - Exploration du tumulus de Tossen-Maharit, commune de Trévérec, Bulletin de la Société d'émulation des Côtes-du-Nord, 37, p. 5-36.

MARTIN A., PRIGENT abbé (1907) - Le Mouden-Bras en Pleudaniel, Bulletin de la Société archéologique du Finistère, 34, p. 146-178.

MICAULT V. (1881) - Essai sur la détermination de l'âge de quelques tumuli de Bretagne, Bulletin de la Société d'émulation des Côtes-duNord, 19, p. 121-163

MORTILLET de A. de (1920) - Les belles pointes de flèches en silex des sépultures morgiennes de Bretagne, Bulletin de la Société préhistorique française, 17, p. 248-255.

MORTILLET de A., DE MORTILLET G. (1881) - Musée préhistorique, Paris, Éd. C. Reinwald, 407 p.

NEEDHAM S. (2000) - Power Pulses Across a Cultural Divide: Cosmologically Driven Acquisition Between Armorica and Wessex, Proceedings of the Prehistoric Society, 66, p. 151-207.

NEEDHAM S., PARFITT K, VARNDELL G., avec les contributions de BIRCHENOUGH A., BUTLER C., CARTWRIGHT C., LA NIÈCE S. (2006) - The Ringlemere Cup: Precious Cups ands the Beginning of the Bronze Age, Londres, Éd. The British Museum Press, 116 p.

NICOLAS C. (2008) - Les pointes de flèches armoricaines du nord du Finistère : étude typologique et technologique d'un bien socialement valorisé de l'âge du Bronze ancien, Mémoire de master 1, Université Paris 1, Paris, 225 p.

PAILLER Y. (2007) - Des dernières industries à trapèzes à l'affirmation du Néolithique en Bretagne occidentale (5500-3500 av. J.-C.), Oxford, Éd. Archaeopress (BAR International Series 1648), 340 p.

PAILLER Y., GANDOIS H., IHUEL E., NICOLAS C., SPARFEL Y (2010) - Le bâtiment en pierres sèches de Beg-ar-Loued, île Molène (Finistère) : évolution d'une construction en pierres sèches du Campaniforme au Bronze ancien, in C. Billard et M. Legris dir., Premiers Néolithiques de l'Ouest : cultures, réseaux, échanges des premières sociétés néolithiques à leur expansion, Actes du $28^{\mathrm{e}}$ colloque interrégional sur le Néolithique, Le Havre, 2007, Renne, Presses universitaires de Renne (Archéologie et culture); p. 415-440.

PRIGENT abbé (1880) - Exploration du tumulus de Porz-ar-Saoz, en Trémel, Bulletin de la Société d'émulation des Côtes-du-Nord, 17, p. 173-194.

PRIGENT abbé (1881) - Fouille du grand tumulus de TossenKergourognon en la commune de Prat, Côtes du Nord, Bulletin de la Société d'émulation des Côtes-du-Nord, 19, p. 15-31.
RENARD C. (2003) - Les armatures de flèches de la fin du IV et du III millénaire dans le Bassin parisien, Mémoire de DEA, Université Paris 1, Paris, $65 \mathrm{p}$.

SALANOVA L. (1998) - Le statut des assemblages campaniformes en contexte funéraire : la notion de «bien de prestige», Bulletin de la Société préhistorique française, 95, 3, p. 315-326.

SALANOVA L. (2000) - La question du Campaniforme en France et dans les îles Anglo-Normandes : productions, chronologie et rôles d'un standard céramique, Paris, Éd. CTHS (Documents préhistoriques 13), $391 \mathrm{p}$.

SALANOVA L. (2007) - Les sépultures campaniformes : lecture sociale, in J. Guilaine dir., Le Chalcolithique et la construction des inégalités, t. 1 Le continent européen, Paris, Errance (Les Hespérides), p. 213228.

SOCIÉTÉ D'ÉMULATION DES CÔTES-DU-NORD (1884) - Trésors archéologiques de l'Armorique occidentale, Rennes-Saint-Brieuc, Leroy-F. Guyon, 8 p.

STEVENIN C. (2000) - Les vases céramiques en contexte funéraire aux débuts de l'âge du Bronze en Bretagne : études typologique, géographique et chronologique, Mémoire de maîtrise, Université Rennes 2, Rennes, 2 vol, 500 p.

TAYLOR J., NODIER C., DE CAILLEUX A. (1846) - Voyages pittoresques et romantiques dans l'ancienne France: Bretagne, Paris, Éd. Didot, 2 vol., 385 p.

TAYLOR J. J. (1970) - Lunulae reconsidered, Proceedings of the Prehistoric Society, 36, p. 38-81.

VORUZ J.-L. (1996) - La chronologie absolue du Bronze ancien, in C. Mordant et O. Gaiffe dir., Cultures et sociétés du Bronze ancien en Europe, Actes du $117^{\mathrm{e}}$ congrès national des Sociétés historiques et scientifiques, Clermont-Ferrand, 1992, Paris, Éd. CTHS, p. 97-164.

WESSEX ARCHAEOLOGY (2009) - Wessex Archaeology. [Disponible en ligne: http://www.wessexarch.co.uk/ (page consultée le 12 mars 2009).]

Clément NICOLAS

Doctorant, université Paris 1 - Panthéon-Sorbonne Université Paris-Ouest - Nanterre-la Défense CNRS, ARSCAN UMR 7041

Équipe Protohistoire européenne Maison de l'archéologie et de l'ethnologie 21, allée de l'Université, 92023 Nanterre cedex clement.nicolas@mae.u-paris10.fr 